Portland State University

PDXScholar

Spring 5-13-2016

\title{
The Use of Anti-Bullying Policies to Protect LGBT Youth: Teacher and Administrator Perspectives on Policy Implementation
}

Michelle Lauren Holliday

Portland State University

Follow this and additional works at: https://pdxscholar.library.pdx.edu/open_access_etds

Part of the Educational Sociology Commons, Education Policy Commons, Elementary and Middle and Secondary Education Administration Commons, and the Lesbian, Gay, Bisexual, and Transgender Studies Commons

Let us know how access to this document benefits you.

\section{Recommended Citation}

Holliday, Michelle Lauren, "The Use of Anti-Bullying Policies to Protect LGBT Youth: Teacher and Administrator Perspectives on Policy Implementation" (2016). Dissertations and Theses. Paper 2921. https://doi.org/10.15760/etd.2917

This Dissertation is brought to you for free and open access. It has been accepted for inclusion in Dissertations and Theses by an authorized administrator of PDXScholar. Please contact us if we can make this document more accessible: pdxscholar@pdx.edu. 
The Use of Anti-Bullying Policies to Protect LGBT Youth: Teacher and Administrator Perspectives on Policy Implementation

\author{
by \\ Michelle Lauren Holliday \\ A dissertation submitted in partial fulfillment of the \\ requirements for the degree of
}

\author{
Doctor of Philosophy \\ in \\ Sociology
Dissertation Committee:
Maura Kelly, Chair
Matthew Carlson
Dara Shifrer
Samuel Henry

Portland State University

2016 


\begin{abstract}
Although in recent years there has been increased attention on bullying prevention and bullying legislation in the United States, there is limited research on the implementation of anti-bullying policies. Moreover, few studies have addressed the use of anti-bullying policies to protect LGBT youth from bullying. The present study seeks to examine the role of anti-bullying policies as a means to protect against bullying based on perceived sexual orientation and gender identity. Qualitative interviews with high school teachers, administrators, and staff members within an urban school district in the United States were conducted to gain insight into how those charged with the task of protecting LGBT youth engage with their school and district policy in efforts to create a supportive environment for their students. In this study, I argue the following: 1) the policy structure, both in the language of the state law and district policy on bullying created barriers for schools to implement the anti-bullying policy; 2) the barriers created by the policy structure limited teachers' ability to protect LGBT youth from bullying; and 3) despite the evident barriers, teachers found ways to create supportive classroom environments for their students. Results indicate that teachers are not knowledgeable of the contents of their school's anti-bullying policy, and have had limited exposure to the policy through training specific to their school's anti-bullying policy. Similar results occurred when teachers and administrators were questioned about their awareness of trainings specific to the prevention of bullying against LGBT youth, posing significant barriers to effective policy implementation. In addition, interview data suggests that although teachers lack the sufficient support in terms of training on the anti-bullying
\end{abstract}


policy, there were multiple examples of teachers serving as advocates for LGBT youth in both their classrooms and in their schools more broadly. The displays of advocacy by teachers, in addition to the presence of district and school administrator support for LGBT students, serve as an example of how school districts can find ways to implement school policies, address bullying in their schools, and raise awareness for the unique experiences of LGBT youth in terms of bullying. 


\title{
DEDICATION
}

This dissertation is dedicated to:

\author{
Corrie Stocking
}




\section{ACKNOWLEDGMENTS}

I would like to take the time to acknowledge and thank the teachers, administrators, and staff members for welcoming me into their lives and allowing me to share their story through this project. I hope that in sharing their experiences we can move one step closer to ensuring that all youth, regardless of their sexual orientation or gender identity, are protected in their schools and free from all forms of bullying. I would also like to thank my dissertation committee members, Maura Kelly, Matthew Carlson, Dara Shrifrer, and Samuel Henry, for the support, encouragement, and constructive criticism that enabled me to create this project to the best of my ability. Lastly, I would like to thank my fiancée, Corrie, for her endless encouragement and support throughout this process. 


\section{TABLE OF CONTENTS}

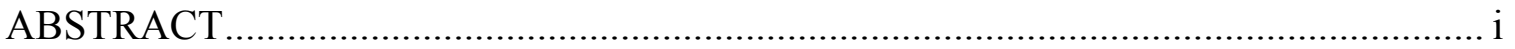

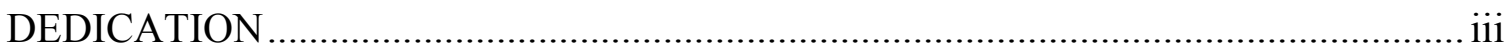

ACKNOWLEDGMENTS …………………………........................................ iv

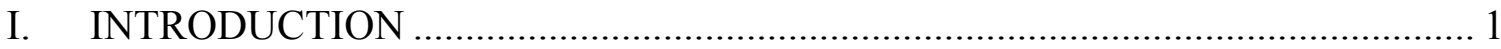

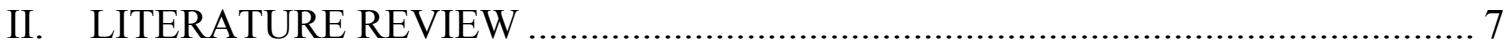

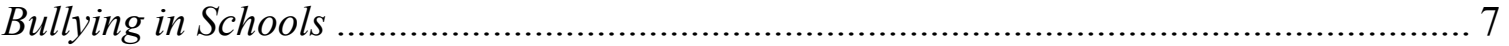

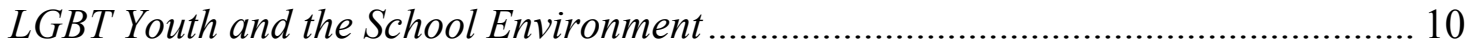

School Policy: The Role of Teachers and Administrators ......................................... 17

Policies Within Communities: The Impact of the Larger Community on Schools ....... 22

Conceptual Framework ...................................................................................... 26

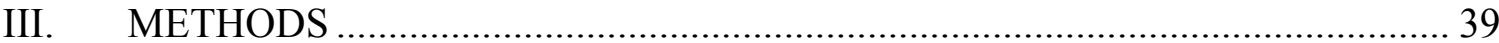

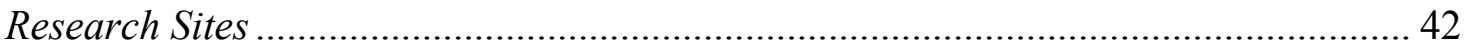

IV. UNDERSTANDING ANTI-BULLYING POLICY: HOW POLICY

STRUCTURE CREATES BARRIERS TO IMPLEMENTATION WITHIN SCHOOLS 46

The Policy Context: State Law, District Policy, and School Policy ............................. 47

Understanding of the Anti-Bullying Policy: Familiarity, Awareness, and Exposure... 51

The Role of Training in Anti-Bullying Policy Implementation .......................................5 58

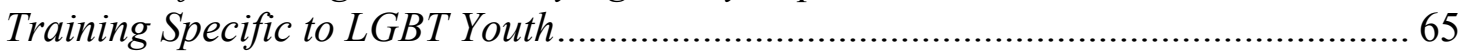

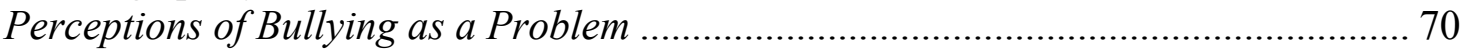

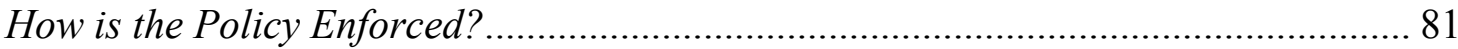

What Works to Prevent Bullying? Policy Effectiveness and Strategies for Prevention 92

V. PROTECTING LGBT YOUTH FROM BULLYING: FACTORS THAT SUPPORT AND PREVENT TEACHERS FROM ADVOCATING FOR LGBT YOUTH ............. 111

Supportive Factors: Teacher Advocacy, Community Building, and Administrative

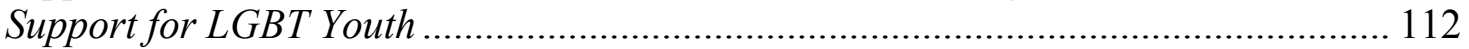

Community-building in the classroom .............................................................. 114

Administrative support for LGBT youth ............................................................. 116

Challenges to Protecting LGBT Youth: Limited Social Networks, Focusing on Race,

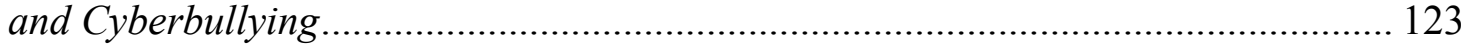

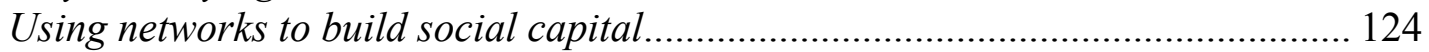

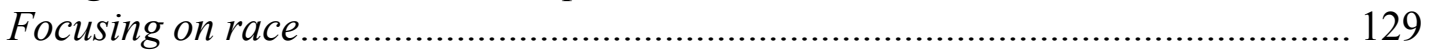

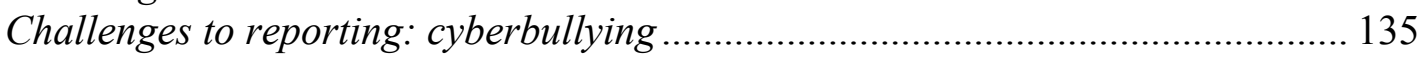

VI. DISCUSSION ………………………………....................................... 139

The Anti-Bullying Policy and Problem Framing in the District.............................. 140

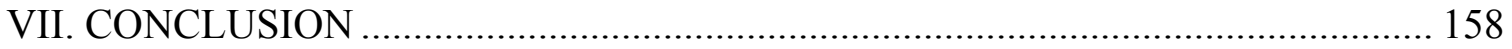

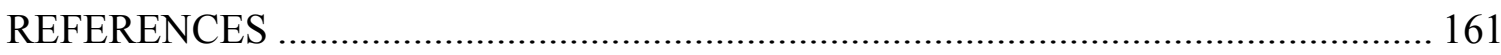

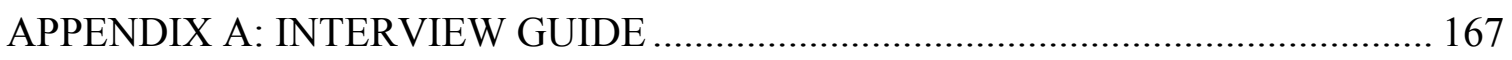




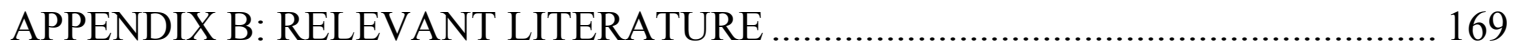

APPENDIX C: STUDY PARTICIPANT CHARACTERISTICS ............................... 172 


\section{INTRODUCTION}

Although reports of bullying have decreased in the past decade, in 2013, over $20 \%$ of 12 to 18 year olds reported being bullied at school (Musu-Gillette et al. 2015). The federal government defines bullying as unwanted and aggressive behavior that is repetitive and includes an imbalance of power between the bully and the victim (HHS 2014). Bullying can be verbal, physical, and/or social, and includes bullying through the internet (cyberbullying) (HHS 2014). In the past twenty years, there has been a marked increase in the attention towards youth bullying in both the media and scientific literature. Swearer, Espelage, and Napolitano (2009) reported a 200\% increase in journal publications regarding bullying has occurred since 1997. In addition to the increase in journal publications, federal departments have increased their support for bullying prevention. The U.S. Department of Education reports an increase in states enacting bullying laws since 1999; in 201021 states created new legislation for bullying prevention (U.S. Department of Education 2011).

Bullying is a challenging issue to address, as general instances of bullying occur but youth are also targeted for individual characteristics. The Department of Health and Human Services identifies lesbian, gay, bisexual, and transgender (LGBT) youth as being at an increased risk for bullying and harassment (U.S. Department of Health and Human Services 2014). The 2013 National School Climate Survey reported over 70\% of LGBT students experienced verbal harassment based on their sexual orientation; over a third of students reported experiencing physical harassment (Kosciw et al. 2014). Despite increased attention and coverage, bullying remains a critical issue for youth across the United States and internationally. National agendas such as Healthy People 2020 include 
LGBT health as a new topic area and prioritize reductions of bullying among LGBT youth.

In the United States, comprehensive strategies to prevent bullying of all youth have been developed (HHS 2014). The prevention of targeted bullying, however, remains scarce. Previous research suggests that creating a supportive school environment can reduce the occurrence of bullying and harassment among LGBT youth (Goodenow, Szalacha and Westheimer 2006; Hatzebuehler et al. 2013; Hatzenbuehler 2011; Marshall et al. 2011; Button, O’Connell and Gealt 2012; Hatzenbuehler, Birkett, Wagenen, and Meyer 2014). A supportive school environment is one in which LGBT youth have access to social support, feel comfortable discussing problems or concerns with teachers and administrators, and are protected against physical and verbal harassment (HHS 2014; Russo 2006; Jacob 2013; Hatzenbuehler et al. 2013; Hatzenbuehler 2011).

Teachers have an integral role in preventing bullying of LGBT youth. Previous research indicates that a teacher's perception of bullying and anti-bullying policies in their school influences whether or not they believe the school is supportive of LGBTQ youth, and if they believe the school is doing enough to protect LGBTQ youth from bullying (Kolbert et al. 2015). Additional studies take this claim a step further, providing support for the role of attitudes and subjective norms in a teachers' decisions to intervene when LGBTQ youth are harassed (McCabe et al. 2013). Teachers' intention to intervene is related to whether they believe protecting LGBTQ youth is important and is sanctioned by colleagues and staff in the school environment (McCabe et al. 2013). While these studies demonstrate that individual factors influence teachers' decisions to intervene in 
bullying, they do not address the role of structural factors, such as school policy, in shaping teacher and administrator engagement with youth who are bullied.

Adoption of anti-bullying policies at the state, district, and school level have been recommended to help create a more supportive school environment for all youth (HHS 2014). There is limited research on the success of anti-bullying policies affecting outcomes for LGBT students. Hatzenbuehler and Keyes (2013) found that lesbian and gay youth in counties with fewer anti-bullying policies protecting LGBT youth were 2.25 times more likely to have attempted suicide in the past twelve months compared to youth in counties with a greater number of inclusive district policies. Including sexual orientation as a protected class in school policies is also associated with lower reports of homophobic remarks and greater reports of teachers intervening when hearing homophobic remarks (Kosciw et al. 2014). Existing data indicates that there are currently 49 states with anti-bullying legislation, however, few states include sexual orientation as a protected class within those laws (LawAtlas 2014). The exclusion of sexual orientation from state laws on bullying reinforces the importance of understanding how anti-bullying policies are being implemented within the United States, and which states are including protections for youth based on their sexual orientation.

An analysis of state anti-bullying policy under the U.S. Department of Education indicated that, by 2010, 46 states adopted an anti-bullying policy (U.S. Department of Education 2011). Of those states, 14 states specified sexual orientation as a protected class (U.S. Department of Education 2011). Of a random sample of 20 school districts, 8 school district policies included sexual orientation as a protected class (U.S. Department of Education 2011). Variation in method of implementation (i.e. teacher training 
programs vs. support groups), and inconsistency in requiring policies be implemented has led to mixed results in the literature regarding the enforcement of anti-bullying policy (Terry 2010; U.S. Department of Education 2011; Kester and Mann 2008).

Understanding how anti-bullying policies are implemented involves assessing the role of teachers and administrators in enforcing the policies. Previous research indicates that teachers and administrators play an important role in how policy is implemented (Coburn, Matia and Choi 2013; Marz and Kelchtermans 2013; Coburn 2001). Qualitative assessments with LGBT youth reveal that teachers and school personnel are often unhelpful and not seen as sources of support in reporting or preventing instances of bullying (Grossman et al. 2009). Similarly, education policy research demonstrates that principals influence how policy messages are framed, while teachers individually and collectively interpret school policy messages, ultimately influencing their decision for or against implementing a policy (Coburn 2001; Marz and Kelchtermans 2013). A recent national study conducted by the Gay, Lesbian, and Straight Education Network (GLSEN) examined the implementation of anti-bullying policies, as well as the effects of antibullying policies on the school climate for LGBT youth in the United States.

Analysis of a sample of over 9,000 public school districts across all 50 states revealed inconsistencies in coverage with some states having a lower proportion of districts with anti-bullying policies compared to others (Kull et al. 2015). In addition, among enumerated categories, race, gender/sex, and religion were the most commonly protected classes $(70 \%, 66 \%$, and $65 \%$, respectively), while only $42 \%$ of districts enumerated sexual orientation and 14\% included gender identity/expression (Kull et al. 2015). Based on the results of their analysis, an in-depth examination a school district 
within one of the states the includes sexual orientation as a protected class in its antibullying policy would be beneficial to understanding the impact of an enumerated antibullying policy on bullying among LGBT youth.

This study aimed to assess the role of anti-bullying policy implementation in the prevention of harassment based on perceived sexual orientation and gender identity in secondary schools. In conducting this assessment, the following questions were addressed: 1) How do teachers and administrators implement anti-bullying policies? 2) What are the views of teachers and administrators of the anti-bullying policy in their district? and 3) How do teachers and administrators perceive the use of anti-bullying policies to protect LGBT youth from harassment? Qualitative interviews with school teachers, principals, vice principals, and staff allowed for an in-depth investigation of the aforementioned questions. In addressing these questions, the present study contributes to the literature on bullying and the school environment by adding a contextual understanding of how district policy translates into school implementation and practice. The role of teachers and administrators in protecting LGBT youth from bullying in their schools were also assessed.

Chapter II provides an overview of the relevant literature on bullying, LGBT youth, and the role of teachers and administrators in the school environment. Chapter II also contains the conceptual framework for this study. In Chapter III, I provide a detailed account of the methods used in the present study. Chapters IV and V are both analysis chapters. Chapter IV begins with an overview of the policy context at the state, district, and school level, in addition to analyzing the role of training, and training specific to LGBT youth, in creating an environment conducive to policy implementation. Chapter 
IV focuses primarily on bullying in general, and also provides the results of examining the structure of the schools in terms of how bullying was framed as a problem by administrators, in addition to how teachers perceived bullying in their schools. References to LGBT youth are included when relevant in this chapter. In Chapter V, I include an analysis of the factors that supported and inhibited teachers' ability to protect LGBT youth from bullying. Teachers found support through their administration, developed strategies in their classrooms to discourage bullying, and relayed on limited social networks to protect LGBT youth in their schools. Chapter V also discusses the challenges teachers faced when seeking to interrupt or prevent bullying based on sexual orientation or gender identity/expression, highlighting the role of the lack of social networks, race as a priority in the school district, and issues related to cyberbullying. Chapter VI includes a discussion of the findings, connections to the existing literature, as well as implications and recommendations. The discussion section is followed by the conclusion (Chapter VII). 


\section{LITERATURE REVIEW}

\section{Bullying in Schools}

Bullying of adolescent youth is a national concern in the United States. Although the current literature illustrates a decline in bullying among adolescents, rates of reported bullying remain remarkably high (Perius et al. 2014). Data from the 2011 National Crime Victimization Survey indicates $27.8 \%$ of students age 12 to 18 experienced bullying on school property (U.S. Department of Education 2013). In an analysis of the 2013 National Youth Risk Behavior Survey, 19.6\% of 9-12 grade students reported bullying on school property (Kann et al. 2014). Approximately 10\% of adolescents in the United States report cyberbullying (U.S. Department of Education 2013; Kann et al. 2014).

Bias-based bullying creates additional challenges in understanding and preventing bullying across the United States. Bias-based bullying refers to bullying based on personal characteristics such as race/ethnicity, sexual orientation, religion, or disability (Russell et al. 2012). Data from the 2013 National School Climate Survey revealed that an overwhelming percentage of LGBT youth report hearing homophobic remarks at school. Over $50 \%$ of students reported hearing negative remarks from teachers and school staff regarding their sexual orientation and/or gender expression (Kosciw et al. 2014). Despite over half of the students reporting LGBT-related discrimination policies in their schools, $17 \%$ of youth reported being prohibited from starting a Gay-Straight Alliance (GSA) and $42 \%$ of transgender youth reported being unable to use their preferred name in school (Kosciw et al. 2014).

Bullying based on sexual orientation or gender identity/expression is further complicated by the increasing significance of cyberbullying in schools. Cyberbullying 
creates difficulties for all students in schools, because cyberbullying is difficult for teachers and administrators to catch, and students may not feel comfortable reporting cyberbullying to an adult in school (Underwood and Rosen 2011; Cooper and Blumenfeld 2012). Cooper and Blumenfeld (2012) conducted an analysis of the affects of cyberbullying on LGBT youth. Results from a quantitative survey of LGBT and allied youth ages 11 to 18 revealed that LGBT youth experienced bullying online through social networking sites, online chatrooms, and instant messaging (Cooper and Blumenfeld 2012). Among LGBT students, $71 \%$ experienced cyberbullying, with $60 \%$ being bullied online because of their sexual identity and 14\% reporting bullying based on their gender identity/expression (Cooper and Blumenfeld 2012). Of the LGBT students who did report being cyberbullied, 56\% stated that they felt depressed because of cyberbullying, $35 \%$ reported having suicidal thoughts, and over $20 \%$ reported that they isolated themselves from friends, were afraid to go to school, and had lower grades because of cyberbullying (Cooper and Blumfeld 2012). The results from their study provide additional support for comprehensive anti-bullying policies that include sexual orientation and gender identity/expression to prevent students from being bullied online, and enable school staff to intervene and protect LGBT students when they are bullied online.

The school environment can be a source of rejection and support for LGBTidentified youth. The role of the school environment in fostering a safe space for LGBT youth is well-documented. A comparative analysis of the relationship between youth harassment, health, and school-related outcomes (i.e., violent threats and grades) revealed that youth who experienced biased-based harassment were more likely to report substance abuse, suicidal ideation in the past month, suicide attempts in the last year and 
worse grades when compared to youth experiencing general harassment (Russell et al. 2012). Harassment based on being perceived as gay, lesbian, or bisexual was among the highest reported types of harassment (Russell et al. 2012). Students reporting harassment based on sexual orientation were more likely to report substance abuse, suicide, and poor school outcomes compared to race, religion or gender-based harassment (Russell et al. 2012). The findings of this study are consistent with previous research and data at the federal level which identify LGBT youth to be at an increased risk for bullying (HHS 2014).

Hatzenbuehler et al. (2013) examined the role of protective school climates (i.e., presence of Gay-Straight Alliances (GSAs), safe spaces, inclusive school curricula) in suicidal thoughts, plans, and attempts in a 12-month period. Through an analysis of Oregon Health Teen survey data for 2006-2008, Hatzenbuehler and Keyes (2013) examined the relationship between suicide attempts among lesbian, gay and bisexual youth (LGB) and the proportion of school districts that had inclusive anti-bullying polices (policies that included sexual orientation in the protected group) at the county level. Hatzenbuehler and Keyes (2013) found that lesbian and gay youth living in a county with a smaller proportion of districts with inclusive policies were 2.25 times more likely to attempt suicide in the past 12 months. There was no protective relationship between inclusive policies and suicide attempts for bisexual youth or heterosexual youth, however, peer victimization was less likely in school districts with inclusive policies (Hatzenbuehler and Keyes 2013). Their findings provide support for the role of the social climate of schools on mental health in LGB youth, in addition to the impact that inclusive policies may have on the degree to which students experience bullying in school. Their 
findings illustrate that the presence of inclusive policies reduce the likelihood of suicide attempts but suggest that additional studies need to be conducted in order to significantly reduce suicide attempts. Hatzebuehler et al. (2013) demonstrate the powerful effect that an inclusive school environment has on LGBT, however, protections at the level of school clubs and curricula are not enough. Politically, despite nondiscrimination policies such as Title IX, schools are continuously faced with the need for complete protections for LGBT youth in the event of victimization (Cianciotto and Cahill 2012).

\section{LGBT Youth and the School Environment}

Limited support through school policies increases the challenges associated with understanding the role of the school environment in protecting against bullying of LGBT youth. While anti-discrimination policies exist at the federal level, the federal government has yet to create an anti-bullying policy in the United States, ultimately leaving the protection of LGBT youth in the hands of state departments of education and local school districts (HHS 2014). In an earlier work, Cahill and Cianciotto (2004) examined the role of federal, state, and policies on creating safer schools for LGBT youth. "No promo homo" policies in certain states across the United States were addressed in the article as they prevent schools from protecting LGBT students. Federal policies such as Title IX and the Equal Access Act provided students and schools with avenues for ensuring that LGBT youth are not discriminated against in the education system. However, Cahill and Cianciotto (2004) highlighted the limitations associated with federal policies as Title IX only holds schools accountable if they are notified of discriminatory behavior. Parental notification laws in states across the United States 
(Arizona, California, Nevada and Utah) required parents to consent before their children can participate in any classes that discuss topics pertaining to sexuality (including AIDS) (Cahill and Cianciotto 2004).

At the time the article was written, some states still maintained sodomy laws. Protections for LGBT students varies by state and local region which shapes school environments and further complicates the ability to provide sanctions in support of LBGT youth. Cahill and Cianciotto (2004) offer the following recommendations regarding safer schools: 1) staff training on how to provide support for students struggling with their sexual orientation or gender identity/expression, 2) inclusive school curricula that identifies the positive contributions that LGBT individuals has made to U.S. history, 3) greater adoption of the Safe Schools Initiative (developed by the Massachusetts Board of Education in 1993) and 4) increased adoption of Gay-Straight Alliances (GSAs) which have been shown to improve the school environment. The review of policies provided in this article are useful for understanding the history and current state of LGBT youth in the United States education system. Continued research in policy and in particular policy enforcement can aid in reducing discrimination and harassment faced by LGBT youth in schools across America. Greater federal involvement is essential to reducing the lack on continuity in policy adopting across the United States. Currently, several LGBT rights advocates are raising support for the passing of the Student Non-Discrimination Act (SNDA). SNDA would provide federal legal protections for LGBT students and prohibit public schools from discriminating against students based on the perceived or actual sexual orientation or gender identity of themselves of anything they associate with (HRC 2016). The bill has been proposed but has yet to pass. 
Analyses of bullying policies reveals few states include protections based on sexual orientation (U.S. Department of Education 2011). In an analysis of anti-bullying policies and the school climate for LGBT youth, Kull et al. (2015) found that LGBT youth who knew their school's policy was inclusive of LGB or LGBT youth were more likely to report bullying or harassment, compared to LGBT students who were not aware that their school's policy was inclusive. In addition, although findings did demonstrate greater reports of teacher's effectively responding to incidents, $41 \%$ of students in schools with inclusive policies characterized teacher's responses as ineffective, highlighting the importance of deepening our understanding of how methods of policy implementation, such as teacher training, impact the school environment for LGBT youth (Kull et al. 2015).

In the absence of formal protections for LGBT youth, many schools have turned to GSAs to mitigate the effects of bullying and provide a more supportive environment. Mayberry, Chenneville, and Currie (2013) provide support for the role of GSAs in creating a safe space for youth but illuminate inherent challenges to school-wide support for GSAs. Mayberry et al. (2013) conducted a qualitative study to assess the effectiveness of GSAs in high school reform. Using the conceptual framework for school change efforts and practices, Mayberry et al. (2013) examined the role of silence in perpetuating heterosexual norms in schools, creating "safe spaces" for LGBT students can feel a sense of belonging and find protection, as well as efforts to create systemic school change through normalizing all forms of sexuality in schools through the curriculum, resources and other practices that promote social justice for LGBT youth. Results of qualitative interviews conducted with GSAs members, sponsors, school 
principals and district administrators revealed a variety of responses. Faculty silence with regard to ignoring homophobic remarks and failing to establish intolerance for antigay remarks in the classroom was one example of silencing (stigmatizing homosexuality and only focusing on heterosexuality) reported in the study. Examples of passive resistance to GSAs in the high school under study included adopting the GSA but no showing overwhelming support for its presence in the school, such as heterosexual students being unaccepting of the GSA but not taking steps to disband the group (Mayberry, Chenneville, and Currie 2013). Students reported GSAs promoted a sense of community and administrators reported GSAs as a method of ensuring student safety. GSAs were also found to foster activism through standing up against antigay statements in the classroom to raising awareness through T-shirt sales. The examples provided by Mayberry et al. (2013) indicate that GSAs are a critical component of challenging the current culture of heteronormativity in American public schools. Their conceptual framework could serve as a practical tool for analyzing the effect that initiatives aimed at protecting LGBT youth have on the culture of the school. Additional research is needed to measure the intent of positive effects that GSAs have on LGBT and heterosexual students. The persistence of faculty silence and passive resistance suggests that additional resources are needed to truly challenge the heterosexual norms.

Qualitative interviews with teachers, administrators, and students revealed GSAs provide social support for LGBT youth; participants described GSAs as fostering activism and raising support against anti-gay remarks in the classroom (Poteat et al. 2013). The effects of GSAs on suicide attempts, plans, sense of school belonging, and harassment based on sexual orientation are less understood (Hatzenbuehler and Keyes 
2013; Poteat et al. 2013, Toomey, McGuire and Russell 2012). Previous studies illuminate barriers to supportive environments for GSAs. Watson et al. (2010) analyzed the factors which prevent or allow GSA advisors to effectively advocate for LBGTQ youth in their schools. Interviews conducted with $22 \mathrm{GSA}$ advisors revealed that sociocultural, school and individual level factors aided GSA advisors in advocating for LGBTQ youth and also presented barriers to advocacy.

Sociocultural factors included external factors such as parents, public policy, cultural norms and community resources (Watson et al. 2010). Parents were reported as a barrier to advocacy in preventing their children from joining a GSA, while parental support served as a facilitating factor in fostering LGBTQ youth self-esteem (Watson et al. 2010). Public policies prevented advocacy in some instances where nondiscrimination policies did not include LGBTQ students as a protected group. Enforcement of LGBTQ protectors were insufficient even when students were included in the protected class (Watson et al. 2010). Societal norms opposing homosexuality created barriers to advocacy and several advisors reported that community resources and support facilitated advocacy efforts, school-based factors included administration, school personnel nonadministrators, students, school policy and school-based resources (Watson et al. 2010). Administrators and school personnel were credited with serving as both facilitators and barriers (Watson et al. 2010). In addition, advisors reported that there was a lack of LGBTQ role models due to school personnel fearing for their jobs if they were to come out (Watson et al. 2010). Individual level factors included consequences to advocacy, sexual identity of advisor, knowledge of LGBTQ issues, personality characteristics and personal experiences, and advisors who identified as heterosexual reported having limited 
knowledge for advocacy efforts but also reported being less fearful of negative consequences given their privileged status (Watson et al. 2010). Overall Watson et al. (2010) demonstrate that there are several barriers to protesting LGBTQ youth using GSAs. Lack of teacher awareness of the GSA, lack of support for the GSA, and insufficient knowledge of advocacy groups in general. The findings of Watson et al. (2010) shed light on areas that schools need to focus on when training their staff, as well as potential avenues for implementing school anti-bullying policies.

As primarily student-led clubs, the institutionalization of GSAs is essential to protecting LGBT youth from hostile school environments (Grace and Wells 2009). An analysis of the Massachusetts Safe Schools Program, implemented in 1993, highlights the role that GSAs have in high schools. Qualitative interviews, questionnaires, and observations with superintendents, teachers, school staff, parents and students across 22 high schools revealed that GSAs provide counseling/support, constitute a "safe space" and serve as the primary medium for raising awareness about LGBT issues in the school (Griffin et al. 2004). Based on the principal's support for the school's GSA, the school was able to incorporate LGBT student rights into the school handbook and used school policy to curb heterosexist remarks made by faculty (Griffin et al. 2004). In a similar study, Fetner et al. (2012) critically examined the concept of a "safe space" through interviews high school students involved in GSAs or similar groups in their school.

The findings of Fetner et al. (2012) exposed several challenges in the school environment, such as hostility towards the GSA by teachers and students (Fetner et al. 2012). The spaces enabled youth who identified as LGBTQ and youth who were not yet ready to disclose their sexual identity to develop a sense of community and protection 
against the homophobic remarks and physical harassment suffered in school (Fetner et al. 2012). Both qualitative assessments reinforce the importance of student-led support groups for LGBT youth and draw attention towards the limitations of GSAs in providing complete protection for LGBT youth. As mentioned by both Fetner et al. (2012) and Griffin et al. (2004), GSAs are met with resistance from administrators in schools and are bound by the existing school policy structure regarding their reach and scope (Fetner et al. 2012; Griffin et al. 2004). Cultural norms that promote heteronormativity, and discriminatory aspects of school policies remain unchanged in the midst of GSA development and activities (Fetner et al. 2012). The prevalence of mixed data on GSAs in high schools draws attention to the need to further develop federal policies in support of protections for youth based on sexual orientation and gender identity. Without protections, LGBT youth may not feel safe enough to report instances of bullying, which could further their feelings of isolation and anxiety/depression. The focus on GSAs and student-led groups, and data on inconsistent adoption of protective polices illuminates the need for a critical understanding of the relationship between anti-bullying policy, policy implementation, and harassment for LGBT youth.

Despite the wealth of research focusing on the impact that the school environment has on bullying of all youth, the social science literature largely focuses on the role of peer interactions in fostering bullying among adolescents (Minton et al. 2008; Mishna et al. 2009; Pascoe 2013). The surplus of studies on peer's role in bullying illustrates their role as both facilitators of bullying and agents in its prevention (Swearer et al. 2010). Critical to understanding the role of peers in bullying is the weight of cultural norms in schools (Thornberg 2011; Swearer et al. 2010; Swearer, Espelage and Napolitano 2009). 


\section{School Policy: The Role of Teachers and Administrators}

At an institutional level, previous research indicates that LGBT youth often report limited support from teachers and school staff regarding intervening when bullying occurs (Grossman et al. 2009). In a qualitative study of LGBT youth perceptions of their school environment, focus groups revealed that students rarely relied on teachers and school personnel to intervene or listen when bullying occurred (Grossman et al. 2009). Participants also reported that teachers would reply to reports of bullying by stating they could not help or stop the instances (Grossman et al. 2009). Kolbert et al. (2015) conducted an extensive study using a 35-item questionnaire to assess if and how teacher perceptions of bullying impact their accounts of bullying of LGBTQ youth. In their study of teacher's perceptions of bullying of LGBTQ revealed that although most teachers stated that their school frequently or always supports all students regardless of sexual orientation, teachers who reported school support of LGBTQ youth from staff and peers also reported perceiving higher levels of LGBTQ youth victimization compared to nonLGBTQ-identified youth (Kolbert et al. 2015). In addition, teachers who themselves identified as LGB felt the students in their schools were less supportive of their LGB peers (Kolbert et al. 2015). Additional results indicated that teachers were often unaware of if their school has an anti-bullying policy, as well as if that policy protects LGBTQ students (Kolbert et al. 2015). Teachers who were unsure if their school's policy protected LGBTQ youth were less likely to believe that their school was doing enough to support LGBTQ youth (Kolbert et al. 2015).

Along a similar vein, Schneider and Dimito (2008) conducted an in-depth survey analysis of elementary, middle and high school teacher's experiences and beliefs about 
discussing LBGT issues in schools. Their sample include both heterosexual and LGBTidentified teachers. Teachers reported witnessing verbal harassment based on real or perceived LGBT identity by both students and teachers, both within and across the two groups (Schneider and Dimito 2008). In addition, the majority of teachers believed the school would protect them if they addressed LGBT issues in schools and had antiharassment policies in their school district, teachers also largely felt that they could access LGBT resources as well (Schneider and Dimito 2008). Despite these positive aspects, the majority of respondents reported little to no exposure to LGBT issues in training or professional development, in addition to reporting that students could not easily access LGBT resources at school (Schneider and Dimito 2008). Several barriers were described toward promoting LGBT issues in schools, mainly parents reactions, as well as fear of being harassed by students and colleagues (Schneider and Dimito 2008). However, over a third of teachers did not believe that the administration would be interested in addressing issues related to LGBT youth, which may explain why few teachers were completely "out" at school(Schneider and Dimito 2008). Schneider and Dimito (2008) represent one of the few studies that exclusively measures the experiences of teachers in schools regarding LGBT youth and safety. Overall, their findings illuminate the role of harassment and school culture, in addition staff training, in hindering adequate resources and support for both LGBT youth and faculty.

Scheider and Dimito (2008) revealed that regardless of sexual orientation or gender identity, teachers are ill-equipped to ensure that LGBT youth feel safe in school and are not at risk for bullying. In a similar study, McCabe et al. (2013) used a Behavioral Intention to Advocate for LGBTQ Youth survey to assess the factors that 
affect a teacher's intention to intervene in the harassment of LGBTQ youth. Results of the survey illustrated that attitudes toward intervening and subjective norms were critical aspects predicting intention (McCabe et al. 2013). Teachers who believed intervening in bullying of LGBTQ youth was valuable and that their colleagues and administrators would support their actions were more likely to report intention to intervene in bullying (McCabe et al. 2013). The findings of Kolbert et al. (2015) and McCabe et al. (2013) demonstrate the importance of not only understanding teachers' perspectives on bullying of LGBT youth, but also the factors that influence whether they intervene in bullying instances, as well as their awareness of a school's anti-bullying policy.

Review of the literature on teachers and school policy demonstrates that examining school policy and implementation is an interdisciplinary and international endeavor (See Appendix B Table I). Internationally, studies from Ireland, Sweden, and Canada lend insight into teachers' perspectives on policy implementation, and their opinions of whether or not bullying is a problem in their school. Questionnaires administered to secondary school teachers in Ireland indicated that the introduction of an anti-bullying program increased both teacher and student perceptions of school safety and ability to intervene (James et al. 2008). The program included school-wide training to increase teacher awareness of bullying as an issue and development of a plan for intervening, reporting, and preventing bullying (James et al. 2008). More than $70 \%$ of teachers surveyed believed the program made an impact, crediting the structure of the program and increased competence in intervening as primary sources of its success (James et al. 2008). 
Although the example from Ireland demonstrates the potential success of a prevention program, examples from Sweden and Canada highlight the role of individual school contexts in creating a successful space for a prevention program. In Sweden, researchers conducted a review of eight schools, in a country where all schools are required to have a program. A mixed methods analysis of a program indicates that schools with a demonstrated commitment to training staff, addressing bullying, and having relationship building activities between teachers and/or students significantly reduced victimization (Flygare, Gill, and Johansson 2013). Canadian studies reiterate the importance of school commitment, as semi-structured interviews with counselors, administrators and teachers revealed that staff were less familiar with forms of technology, were aware of some instances of cyberbullying, did not have a cyberbullying policy and felt the district policy needed improvement (Cassidy, Brown and Jackson 2012). Similar results of a study from Toronto indicate that service providers for LGBT youth identify institutional factors such as inadequate training for educators, lack of equity-based policies and not holding staff and students accountable under existing policies as fostering bullying (Mishna et al. 2009). Federal policies and community level support were cited as sources for mitigating bullying (Mishna et al. 2009).

While previous studies illustrate the importance of teachers' perspectives on policy and intervening in bullying, in the United States, the role of teachers in bullying policy implementation has not been well-documented. Research indicates that principals and professional development meetings are integral in teacher's decisions to implement a policy (Coborn 2001). Greytak, Kosciw, and Boesen (2013) conducted an analysis of professional development meetings and an increased skillset for preventing bullying of 
LGBT youth. Results of two-hour professional development training sessions among teachers, principals, vice principals, school staff, and mental health professionals revealed variation in awareness of bullying and increased competence post-training (Greytak, Kosciw, and Boesen 2013). Principals and vice principals were the least knowledgeable about instances of bullying but were more likely to report high levels of self-efficacy in intervening and addressing bullying of LGBT youth (Greytak, Kosciw, and Boesen 2013). The trainings resulted in increased empathy for LGBT youth, perceptions on the importance of intervening in bullying, and increased self-efficacy regarding intervening among teachers (Greytak, Kosciw and Boesen 2013). These findings provide support for the role of professional development meetings in improving the school environment for LGBT youth and teacher perspectives on bullying of LGBT youth.

In addition to teacher perceptions and training, studies suggest that the hierarchy of power in education systems influences teachers' social networks and how policy is understood and implemented (Bauman and Del Rio 2006; Bauman, Rigby, and Hoppa 2008; Boulton 1997; Cassidy, Brown, and Jackson 2012; Coburn, Mata, and Choi 2013; Desimone 2006; Greytak and Kosciw 2014; Kennedy, Russom, and Kevorkian 2012). A longitudinal study conducted by Coburn, Mata and Choi (2013) demonstrates the influence of district policy on the gathering of information and development of teachers' social networks in school. The adoption of a district policy to reform the math curriculum influenced teachers in the following way: 1) district policy determined the information that teachers would receive from their colleagues regarding the curriculum, 2) district policy created professional development meetings to increase their level of expertise, and 3) district policy informed and shaped the development of teacher's social networks by 
created a set of "expectations" about how teachers interact with the policy, each other and the curriculum (Coburn et al. 2013). Regarding expectations, teachers often attended district-sponsored professional development meetings in which the meeting leaders would tell the teachers about district expectations for their teaching curriculum (Coburn, Matia, and Choi 2013). Their findings provide support for increased qualitative assessments of teachers/administrators understanding of policy and how the flow of information from district officials to school teachers and staff may impact perceptions of policy and its delivery.

\section{Policies Within Communities: The Impact of the Larger Community on Schools}

One major factor affecting the perceptions of district/school administrators and ultimately the student body is the community context in which school policies are created and schools exist. When considering the complexities of the school environment, school policy, and LGBT youth, it is also important to remember that schools exist in the context of their larger communities. Schools do not exist in isolation, and the values, norms, and expectations established within a larger community can set the tone for the norms and expectations within a school (Coleman and Hoffer 1987). In some communities, the schools are comprised of students that reside in the community, while at others there is a mixture of students who live in the community and those who do not. The changes in access to schools, public versus private schools, and the amount of diversity within schools have created school systems with competing values (Coleman and Hoffer 1987).

As a result, the diversity of values can create challenges for school administrators, who are charged with the task of establishing their authority, and often times creating a 
dominant set of values for the school (Coleman and Hoffer 1987). Considering the existing literature on the impact of teachers' beliefs on their decisions to enact school policies, it is reasonable to suggest that the community values and norms could influence how school policies are perceived by school administrators and teachers.

Previous research on the role of communities in school dynamics indicates that the demographics and values within a community (i.e., socioeconomic status and religious beliefs) can impact how students interact within schools, the value that schools place on extracurricular activities or academics (Coleman 1961; Booth and Gerard 2014). Studies also indicate that the environmental factors within a school have a significant affect on how students perceive their social environment, and whether or not students feel a sense of belonging or connectedness while in school (Booth and Gerard 2014). When applied to LGBT youth, these factors combine to create a "gender climate" where the institutional factors in the school, school policy, and the individual beliefs of teachers and students, can reinforce values that exclude and police the behaviors of LGBT youth (Ullman 2014). Each of these factors are critical to understanding how schools and their surrounding communities, encourage or prevent schools from protecting LGBT youth from bullying.

Coleman (1961) conducted an assessment of the impact of communities on school dynamics. Analyses of a sample of ten schools in rural, urban, and suburban environments revealed that community values (i.e. religion), socioeconomic status, and political orientations impacted not only the resources available to students at schools, but also the composition of the schools and the values that parents and teachers placed on the youth in the community (Coleman 1961). For example, in Farmdale, a small rural 
community, the strength an influence of the Baptist churches resulted in schools not being allowed to hold or sponsor school dances (Coleman 1961). Within one of the larger suburban communities, the socioeconomic status of the residents enabled the community to build a state of the art high school (Coleman 1961). For the students, factors such as whether they had a car, the neighborhood they came from, along with their personality and reputation, impacted their status and friendships within the school as well (Coleman 1961). The results of this study provide support for the impact that community dynamics have on a school. Recognizing these community differences is an integral part of understand if and how a school's anti-bullying policy is received and implemented.

The strength of communities lies in their ability to shape students' perceptions of themselves and others. Existing studies suggest that the degree of fit between a students' characteristics and the characteristics of their social environment (Eccles et al. 1993; Booth and Gerard 2014). Outside of school, students' communities are impacted their neighborhood, surrounding city, and their families (Coleman and Hoffer 1987; Rosser et al. 2000). Each of these environments affects the resources and attributes that students bring with them to their school environment. If students' social environments do not meet their needs, empirical studies suggest that they do not perform well in school (Eccles et al. 1993; Booth and Gerard 2014). In contrast, when students do feel supported by and connected to their social environment, they have positive perceptions of themselves (Booth and Gerard 2014). Through an analysis of the relationship between school climate, students' self-evaluations, and school connectedness, Booth and Gerard (2014) found that there was a relationship between students' attitudes and school climate. High school students demonstrated greater self-efficacy compared to middle school students, 
and whether or not students enjoyed school had an affect on their academic self-efficacy (Booth and Gerard 2014). Their findings did reveal gender differences, with girls reported greater feelings of school connectedness at the beginning of the academic year compared to boys (Booth and Gerard 2014). Boys reported greater self-efficacy than girls during the fall of the school year (Booth and Gerard 2014). The results of Booth and Gerard (2014) provide additional support for the role of the school environment on students' evaluations of themselves, as well as the role that gender may have in affect how students perceive their school environment.

Understanding the school climate as it relates to gender is a critical aspect of understanding school anti-bullying policies. Coined the "gender climate", Ullman (2014) investigated the role of how schools' address gender expression, through organizational rules, and social rules that govern students' physical appearance and send messages about appropriate behavior regarding gender and gender expression. Ullman (2014) sought to examine how schools enforced and maintained gender boundaries for their students. Ullman (2014) conducted qualitative interviews with five LGBTQ youth, ages 16-19, that were attending high school, university or working in Australia. Results indicated that the policies requiring school uniforms reinforced gender boundaries as girls were only allowed to wear skirts (Ullman 2014). Participants reported teachers informally enforcing restrictions on gender expression through judgmental or policing remarks about girls wearing short hair (Ullman 2014). Students also reported that teachers condoned bullying based on gender expression and perceived/actual sexual orientation as evidenced through a lack of response from teachers when students were bullied (Ullman 2014). Even when teachers did intervene, students attributed their support to individual interests in the 
LGBTQ community (Ullman 2014). Students also reported that their schools lacked instruction on issues related to diversity of sexualities or gender expression, and their interactions with their peers confirmed the dominant heteronormative values of the school through the use of homophobic language employed to policy behaviors and shame students for stepping outside of the gender boundaries (Ullman 2014). The fact that participants were at both single-sex and co-educational schools highlights the importance of ensuring that schools are discouraged from excluding sexual and gender minorities from the normative values of the school. The lack of structural support for students and use of school policy to reinforce harmful gender boundaries provides additional support for the importance of enforcing school policies that promote equity and inclusion.

\section{Conceptual Framework}

The complexities of the school environment reinforce the need to develop a theoretical orientation for understanding bullying, policy, and policy implementation. In particular, previous studies suggest that LGBT youth are a difficult group to protect, since few policies exist to protect against bullying based on sexual orientation. Although there are limited theoretical approaches to school policy implementation in the sociological literature, the proposed study draws upon sociological theories, including education, that focus on interactions between students, as well as power dynamics within the school system (i.e., students, teachers, and administrators). Central to the conceptual framework is the framing of the problem of bullying, as well as the role of the administrative hierarchy in schools, including the role of the district superintendent, principals, vice principals and teachers. The dynamics of this hierarchy are important to 
fully understand the role that power and influence have on information dissemination to schools and how teachers use the information they are given (Coburn 2004).

The distribution of resources and information are affected by the hierarchy and are a reflection of the role of school culture in policy implementation. School culture refers to norms, values, traditions, and informal expectations that influence how teachers, administrators, and students form their beliefs and make decisions about their behaviors while in school (Peterson and Deal 2011). Both schools and classrooms function as communities, each with its own unique characteristics. The community element of schools is an important piece in understanding how policies are interpreted, and also if and how policies get implemented. In terms of bullying, school culture encompasses how beliefs about bullying impact how bullying is addressed if it occurs within a school, and the role that teachers, students and administrators play both in its occurrence and prevention. Teachers have a significant effect on the school environment for students. In their classrooms, teachers set the tone for the culture of the class, as well as the norms and values that either supported or discouraged by students. Their attitudes towards bullying, and LGBT youth, could affect how LGBT youth are treated in the classroom, and if students feel safe or protected from bullying while in the classroom. In an assessment of teacher and staff perceptions of bullying, Holt, Keyes, and Koenig (2011) found that elementary, middle, and high school staff varied greatly in their perceptions on bullying, and their beliefs about the climate of their schools. Among participants, 27 percent believed that "a little bit of teasing doesn't hurt." Almost all participants did not believe that students would intervene when witnessing bullying, but did feel adults would step in (Holt, Keyes, and Koenig 2011). Over a quarter of respondents also reported 
witnessing teachers make fun of students in school (Holt et al. 2011). When asked questions about reporting, results indicated that although high school staff felt their school was equitable, they were less likely to intervene when hearing students make inappropriate jokes toward one another (Holt et al. 2011). Their inconsistencies in reporting may have been due to their beliefs that a small amount of teasing was acceptable.

The findings of Holt et al. (2011) illuminate the importance of understanding how teachers manage their classrooms in terms of bullying, and LGBT youth. Rodkin and Gest (2011) suggest that how teachers organize their classrooms can have a positive effect on students' social interactions with one other, and eliminate the hierarchies between students that can foster aggression or bullying. From disrupting negative social behaviors to choices regarding how to organize their students in the classroom, Rodkin and Gest (2011) believed that teachers who were mindful of how classroom dynamics affect peer relationships, and teachers who were actively involved in addressing social issues in the classroom, could create social dynamics that promoted more equitable treatment between students. Instructional and emotional support were referenced as ways to foster positive relationships between the teacher and their students.

Additional students suggest that the teacher-student relationship is an integral part of preventing bullying in the classroom. Doll et al. (2011) argue that the classroom can either be a safe place for students or a place where students do not feel supported. When teachers are unaware of bullying occurring in their classroom, do not intervene, or are slow to intervene, it can send a message to students that the teacher is not available as a source of support or protection (Doll et al. 2011). In contrast, when teachers are readily 
available, students do feel safe in the classroom. Frey et al. (2011) argues that there are several strategies that teachers can employ in their classrooms to mitigate the effects of bullying. In their opinion, the norms and relationships that exist in the classroom are embedded within the larger norms in the school, as well as the support available for staff and students within the school as a whole (Frey et al. 2011). The student-teacher relationship is mutually reinforcing, with students affecting the teacher through their participation in the classroom, and their behavior in the classroom (Frey et al. 2011). Regarding teachers, they have the opportunity to create a structure in their classroom that discourages bullying. Frey et al. (2011) suggest that teachers can accomplish this through both formal and informal forms of instruction. In terms of formal forms of instruction, setting classroom rules that outline the expectations for behavior can provide a safe environment for students (Frey et al. 2011). In addition, the lessons that teachers use in the classroom can teach students about empathy or ethics, which are important aspects of positive social interactions (Frey et al. 2011). Informally, Frey et al. (2011) describe the use of scaffolding techniques such as teaching students to resolve issues in the classrooms themselves, manage conflicts, and ultimately learning how to engage in positive behaviors with their peers.

When applied to LGB youth, the suggestions of Frey et al. (2011), and Holt et al. (2011) indicate that teachers' beliefs regarding LGBT youth, and their responses to LGBT youth being bullied in the classroom, may have a negative affect on the classroom environment for LGBT youth. Previous literature describes the climate of the classroom for LGBT youth as cumbersome. Heternormativity in schools has a negative impact of the visibility of LGBT youth, but also the degree to which LGBT youth feel supported in 
their school. Macgillivray (2000) argues that the culture of heteronormativity, heterosexist ideals and heterosexual privilege spills over in the American education system as GLBTQ youth are excluded from social aspects of education (prom king/queen elections, heterosexual teachers openly expressing their sexuality). In addition to social exclusion, GLBTQ students are excluded from the curriculum (Macgillivray 2000). Positive role models, messages, and images about LGB people are absent from school classrooms (Macgillvray 2000). Sexual orientation may not be openly discussed in schools either. Macgillivray (2000) argues for full inclusion of GLBTQ youth through teaching about sexual orientation and gender identity and non-oppressive social relationships (allowing GLBTQ student clubs). Macgillivray provides useful suggestions for reducing the effects of heteronormativity in public schools and also highlights the importance of cultural context in addressing the inequalities experienced by LGBTQ youth. The relevance of the claims presented in this article 13 years past its publication suggest that increased efforts are needed to provide supportive environments for LGBTQ youth. Teachers can interrupt these norms by creating a culture of respect for all students in their classrooms, regardless of sexual orientation or gender identity. School policies can facilitate teacher support for LGBT youth and encourage teachers to find ways to support LGBT youth in their classrooms, which would require all teachers to receive adequate training on LGBT youth issues.

In order to understand this dynamic relationship between policy implementation, and bullying, the current study draws from social movement literature on framing as well as sociological theories of sense-making. Framing refers to the understanding of schema that both individuals and groups use to perceive the world around them (Benford and 
Snow 2000). Benford and Snow (2000) describe collective actions frames as a set of action-oriented beliefs that are used by organizations to mobilize people and gather support for a specific action. For the purposes of this project, collective action frames are a useful theoretical concept; collective action frames are also used to frame problems and influence the way that policy actors interpret the information presented (Benford and Snow 2000; Snow et al. 1986). How administrators and teachers chose to implement policies in their schools is critical to understanding how LGBT youth are protected using anti-bullying policies.

Regarding sense-making, Spillane, Reiser and Reimer (2002) developed a comprehensive framework for sense-making in policy implementation. Their framework focuses on individual cognition, situated cognition and the role of representations (Spillane, Reiser and Reimer 2002). Individual and situated cognition are concepts that refer to individuals act as sense-makers, interpreting stimuli based on prior beliefs and experiences (Spillane, Reiser and Reimer 2002). Situated cognition was an important concept for the present study, as the authors suggested that individuals interpret information in the context of their surroundings (Spillane, Reiser and Reimer 2002). Coburn (2001) contributes to the concept of sense-making, developing a theory for collective sense-making. Coburn (2001) draws from institutional theory, which posits that messaging shapes actions through regulatory, normative, and cognitive pressures (Coburn 2001). Collective sense-making occurs as individuals construct and interpret messages through social interactions. Analyses of elementary school teachers and a principal support these claims. Semi-structured interviews and classroom observations indicate that principals serve as the primary leadership personnel on reading instruction, framing 
messages about reading as a problem and determining how and what messages regarding the reform policy would be passed on to teachers through professional development meetings and training (Coburn 2001). Collective sense-making occurred as teachers often discussed the policy with one another, and decided which messages to reject/accept based on their preexisting beliefs about teaching (Coburn 2001). The role of principals and administrators in problem framing aligns with the overall level of influence that they have within a school. Conversely, teachers have very little influence within a school (Ingersoll 2003). Using data from national and international surveys on schools and decision making, coupled with field data from four schools in Philadelphia, Ingersoll (2003) analyzed the hierarchical nature of schools, districts, and decision-making within education systems. Results indicated that teachers had the most control when it came academic instruction, and very little control in terms of creating school policies (Ingersoll 2003). Principals perceived themselves as having a significant level of influence on the decisions made within their schools (Ingersoll 2003). When the size of schools was considered, principals had the most influence when it came to setting discipline policies and evaluating teachers at large public schools (Ingersoll 2003). The findings of Ingersoll (2003) contribute to the understanding of how sense-making occurs within schools and how teachers and administrators experience school decision-making in different ways. Sense-making creates a significant contribution to our understanding of teachers' and administrators' decisions regarding policy implementation. Marz and Kelchterman (2013) suggest that as individual and collective sense-making occurs, teachers develop interpretations of school policies that create varied approaches to policy implementation. Interviews with twenty high school mathematics teachers in Belgium revealed that 
interpretations of the school curriculum resulted in supporters or opponents of the reform. However, for both groups, teachers interpreted the reform in the context of their values and beliefs regarding math instruction (Marz and Kelchtermans 2013). Supporters believed that the new program directly reflected their beliefs of how mathematics should be taught; opponents actively opposed the new curriculum and viewed the reform as damaging to the educational curriculum (Marz and Kelchtermans 2013).

Empirically, previous studies provide support for the relationship between problem framing and policy implementation in schools. Coburn (2006) conducted an analysis of a reading instruction initiative in California. Data was collected through ethnographic accounts of informal and formal observations of teacher meetings and classroom instruction over the course of one academic year; interviews with teachers, administrators and school principals were also conducted (Coburn 2006). Support for the reading instruction program was garnered through repeated framing of the need for improved reading comprehension based on low and declining reading scores across within schools (Coburn 2006). Professional development sessions encouraged and enabled faculty to continuously engage in the framing process, as well as begin to reframe the problem in their own school (Coburn 2006; Spillane, Reiser, and Reimer 2002; Snow et al. 1986).

Results of qualitative interviews demonstrated that problems with reading comprehension or standardized testing were repeatedly framed as a result of multiple social interactions between teachers and principals at meetings (Coburn 2006). For example, the definition of the reading problem as due to inconsistent instruction led one school to hire a professional development provider and train teachers on new strategies 
(Coburn 2006). Findings also indicated that the principal was the most influential person in problem framing due to their authority in the school and ability to influence teachers' ability to gain support for the problem from their peers (Coburn 2006). Teacher's interactions with principals allowed them to become active in deciding how to frame the problem of reading and eventually promote implementation of the reading instruction policy (Coburn 2006).

In an earlier work, Coburn (2004) examines the impact of teacher's connection to their institutional environment on practice in the classroom. In-depth interviews with three elementary school teachers, Coburn (2004) assessed teacher experience with institutional pressures to conform to a new type of reading instruction. An analysis of teacher responses to institutional "messages", including school policy, included the following codes: 1) degree of congruence between messages and teacher's preexisting beliefs, 2) degree of intensity in opportunities to engage with messages, 3) degree of pervasiveness in pressure to comply and 4) degree of voluntariness in how messages aligned with mandates or norms (Coburn 2004). Results indicated that teachers were more likely to implement the changes to their reading instruction if the messages they received about the program supported their preexisting beliefs about instruction (Coburn 2004). For degree of intensity, teachers were more likely to use the new instruction method if they encountered institutional pressure through professional development and engaging in skills training with colleagues (Coburn 2004). Remarkably, normative pressures through messages about "what teachers should do" and which modes of instruction are "best" had a greater influence on teachers than regulative pressures (i.e., policy), reinforcing the importance of social norms in adoption of new practices within a 
school. Previous literature demonstrates that teachers' understanding of a policy and belief in its value are essential to their role in implementation (Marz and Kelchtermans 2013).

In addition to using a frame analysis, the concept of social capital lends insight into the relationship between social networks, resource distribution, and the interpretation of information. Previous literature suggests that teachers' professional communities influence what information is passed on regarding school reform and who teachers interact with in the reform process (Coburn, Matia, and Choi 2013). Social capital refers to the resources associated with a network consisting of institutionalized relationships (Bourdieu 1986). A key aspect of these resources are that they are embedded in and maintained by the institution. The network of social relationships is important for the institution and is thus reproduced (Bourdieu 1986). In an analysis and application of social capital in the context of schools, Coleman (1988) defines forms of social capital. Information channels and social norms are two forms of social capital crucial to theorizing policy implementation and school bullying. Since social interactions are a critical aspect of social capital, the flow of information between individuals is important to maintaining social networks in an institution (Coleman 1988; Smylie and Evans 2006).

In the case of policy, a new school policy requires teachers and administrators to gain new skills and knowledge. Effective and open avenues for communication help to facilitate change and growth among teachers and administrators. Coburn and Russell (2008) posit that social capital refers to the resources available to teachers that allow them to create a normative environment in their school that supports changing their teaching practices based on a new policy. Qualitative interviews and a quantitative survey 
of elementary school teachers revealed that the content of professional development meetings, over time, constructed who teachers felt were experts in the new policy, how teachers interacted with each other in their social networks, and the level of trust teachers felt regarding sharing information with one anther about the policy (Coburn and Russell 2008). In addition, the use of coaches, teacher-elected faculty that relayed messages about the district policy as well as trained teachers on the new method of instruction, influenced teacher's social networks, their perceptions of the new policy information, ultimately forming their social capital and competence to implement the new school policy (Coburn and Russell 2008). Coburn and Russell (2008) illustrate the relationship between the structure of a teacher's work environment and their social capital. In a similar study, Gallucci (2003) conducts an in-depth assessment of the relationship between teacher communities of practice and implementation of a reform policy. Interviews and classroom observation of three teachers at elementary schools in the United States resulted in the delineation of two specific communities of practice.

Strong and weak communities of practices substantially impacted how and if teachers implemented a policy (Gallucci 2003). In strong communities of practice, teachers worked together in teams to design their new instruction strategies, in contrast to weak communities of practice in which teachers had minimal influence on one another (Gallucci 2003). Newer teachers, often categorized in weaker communities of practice, adhered greatly to the district-mandated curriculum (Gallucci 2003). Teachers in strong communities implemented the policy in a way that focused on meeting the needs of their students, even if that meant omitting different modules from the new curriculum 
(Gallucci 2003). Both Coburn and Russell (2008) and Gallucci (2003) illuminate the importance for sociocultural factors in teacher's decisions to implement school policies.

Applying the frame analysis to bullying policy, it is apparent that issues of authority and problem framing are essential to influencing if and how teachers chose to implement a school policy. Following Coburn (2004), results suggested that teachers would be more likely to intervene in bullying, and promote anti-bullying policies if they understood and agreed with the policy (James et al. 2008; Grossman et al. 2009). Understanding a school anti-bullying policy, however, is directly tied to a teacher or principal's interpretation of the problem of bullying (Spillane, Reiser and Reimer 2002). Recalling Bourdieu's concept of social capital, it is also evident that how information is transferred between teachers and administrators significantly impacts how teachers understand the policy itself.

As illustrated by a study conducted by Anagnostopoulos et al. (2009), teachers often employ gendered and cultural narratives regarding victims of gender-based bullying in the absence of coherent language in anti-harassment policies. Teacher beliefs about bullying are an important aspect of policy implementation. In order to understand the bullying climate in schools, Holt, Keyes, and Koenig (2011) conducted a study of educators in primary and secondary schools within the United States. Using questionnaires, researchers examined multiple aspects of the school environment, including staff/teacher attitudes regarding bullying, as well as their awareness of instances where teachers or students made disrespectful remarks towards others (Holt, Keyes, and Koenig 2011). Although the majority of staff stated that they would accept LGB students, they also reported observing teachers make fun of students for the way 
that they talked or acted, or looked (Holt et al. 2011). Over half of the respondents believed that students would not intervene if they heard another student being bullied, and that adults would intervene (Holt, Keyes, and Koenig 2011). When the researchers made comparisons across schools, they found that high school staff were less likely than elementary school staff to intervene when they heard inappropriate jokes (Holt et al. 2011). This finding is particularly surprising, given that a high school staff were more likely than elementary or middle school staff when it came to perceiving their school as an equitable environment (Holt et al. 2011). The findings of Holt et al. (2011) are important to our understanding of sense-making, because teachers already come into the school setting with a variety of individual beliefs, all of which may have a positive or negative effect on how they interpret school policies.

Structurally, the flow of resources and information are influenced from the top at the administrative level (i.e., district superintendents) down to teachers (Desimone 2006; Coburn et al. 2013). In an assessment of differences among teachers and administrators, Desimone (2006) conducted an extensive quantitative study measuring teachers', principals', and district leaders' perceptions of their policy environment. The results indicated that while teachers and administrators agree regarding barriers to implementing school policies and district standards, there is considerable disagreement on the understanding and implementation of standards, in addition to differences between reports of district authority (i.e., district creates its own standards, etc.) (Desimone 2006). The variability between groups of authority within the education system highlight the need to examine each group's perception of the anti-bullying policy. 


\section{METHODS}

The present study used semi-structured interviews to understand teachers' and administrators' perspectives on the role of anti-bullying policy as a means to prevent harassment based on perceived sexual orientation and gender identity (i.e. lesbian, gay, bisexual, and transgender youth) in high schools. The use of the qualitative interview method allowed for capturing teacher and administrator accounts of their personal experience with the implementation of an anti-bullying policy in their school. For this

study, the sample was restricted to principals, vice principals, school psychologists, and teachers currently employed at public high schools in a large urban school district in the United States.

The high schools were within one large urban school district in the United States. The school district is one of the largest school districts in its region. Compared to the surrounding city, the school district was diverse racially, ethnically, and in terms of socioeconomic status. Thus, this district offered a unique opportunity to explore how a large urban school district uses its anti-bullying policy to protect LGBT youth. Teachers and administrators were chosen as the focus of this project based on previous research suggesting that LGBT youth often report limited support from teachers and staff regarding intervening in instances of bullying (Grossman et al. 2009). In addition, qualitative research studying LGBT youth revealed inadequate training for teachers, and a lack of equity-based policies which included sexual orientation as contributing factors for bullying in schools (Mishna et al. 2009; Anagnostopoulos et al. 2009). Principals and vice principals were included because they influence teachers' understanding and enactment of school policies (Coburn 2006). Teachers were included in the study because 
they are directly involved with students, may witness acts of bullying, and are responsible for reporting incidents of bullying under the state policy. School psychologists were included based on previous research connecting school counselors and psychologists to the school GSA (McCabe et al. 2013).

For the current study, purposive sampling was used to identify teachers and administrators at public high schools that currently have an anti-bullying policy (Marvasti 2004). The population was restricted to teachers and administrators at 9 of the 10 high schools in the district. One high school was excluded from the study as it was a school for non-traditional youth, ages 16-21, who were nearing graduation. Recruitment of participants occurred through email. Since each school had a student club specific to LGBT and/or queer youth, teachers that were listed as club advisors were also recruited for an interview. Emails were obtained from the public state and public school district directories. Participants were identified through snowball sampling of teachers, principals, vice principals, and staff. Email and snowball sampling recruitment resulted in 21 participants from five schools being identified and interviewed for the study.

The participant sample consisted of 2 principals, 3 vice principals, 15 teachers, and 1 school psychologist. Interview participants were clustered by school to ensure a comprehensive understanding of how policy is implemented across a school district, position to understand differences between teachers/staff and administrators, and by years in school to understand if years in school affect perspectives on bullying and policy implementation. Interviews were conducted between January and March of 2016. The participant interviews were conducted face-to-face using a semi-structured interview guide (see Appendix A) with an average interview time of 25 minutes due to the time 
restraints of the participants. Pseudonyms were given to each participant and created for each school to ensure confidentiality. Participants, on average, had worked in the school for 4.5 years, ranging from serving in their first year at the time of interview upwards to fourteen years working at the school. In total, there were five schools, Avenue High School (7 participants), Bayview High School (5 participants), Central High School (4 participants), Dover High School (2 participants), and Eastpointe High School (3 participants). Each school had a GSA, Queer-Straight Alliance (QSA), or similar LGBTQ student-led school club. Table II (Appendix C) provides additional details on the participants at each school).

The current study used an inductive approach to data analysis. The advantage to the inductive approach is employing a method that allows for the synthesis of raw data into summary format through the analysis of emergent themes, in addition to establishing links between the findings and generating a theory or model about the nature of the experiences outlined in the interviews (Thomas 2006). Interviews were coded by use of themes identified in previous literature, and emergent themes in the data. After being audio-recorded and transcribed verbatim by the researcher, analysis of emergent themes in the data occurred through the use of Dedoose software. Each transcript was uploaded to Dedoose and coded with each code corresponding to a segment of text. Word-by-word and focused coding techniques were used to analyze each transcript individually and in relation to the other interview transcripts (Charmaz 2006). The analysis of participant data focus on the following 12 topics discussed in the interviews: bullying as a problem in the school, challenges and issues with reporting of bullying, classroom culture and bullying, discussion of bullying with other teachers/admin/staff, how incidents are (were) 
handled, familiarity with the policy, awareness of the policy, reactive responses to bullying, training, and perceptions or perspectives on how to protect against bullying and harassment. When considered in the context of the existing literature, the 12 topics were organized into two main themes: 1) the role of policy structure in bullying prevention and policy implementation, and 2) what factors supported or inhibited teachers from protecting LGBT youth from bullying in their school.

Copies of the district policy were used to assess adherence to the state law. Regarding the state, district, and school anti-bullying policies, copies of the policy were accessed online through local websites. I reviewed each policy and noted key definitions (i.e. bullying, harassment, sexual orientation, and gender identity) as well as specific language either requiring or encouraging districts to create their own policy, how to report incidents, and language around implementation. Additional information regarding the policies is included at the beginning of Chapter IV.

\section{Research Sites}

Interviews were conducted at one of five schools within the district: Avenue High School, Bayview High School, Central High School, Dover High School and Eastpointe High School. The surrounding city in the district had a demonstrated commitment to the LGBTQ adult and youth population. Each school had unique characteristics in terms of demographics, the visibility/role of the LGBT student group, and the commitment/support of teachers and administrators regarding bullying, the anti-bullying policy, and LGBT youth. The following paragraphs will describe the characteristic of the schools used in this study. 
Avenue High School was the most affluent school in the study population, very few students qualified for free and reduced-price lunch in the school. Racially, Avenue High was a majority white school, and the QSA was described by several participants as thriving or robust. The administration at Avenue High was very supportive of LGBT youth. One staff member was very instrumental in ensuring that LGBT youth were included in anti-bullying programs in the school. Students were recognized in the school for their commitment to bullying prevention, and the QSA was actively involved in antibullying campaigns. In terms of the school anti-bullying policy, Avenue High had a link on their school's website that led to their student handbook.

Bayview High School was similar to Avenue High School in its demographic makeup and administrative commitment to bullying and LGBT youth. The school had a mixture of very affluent and lower socioeconomic status students but Bayview High was also a majority white school. One teacher described the LGBTQIA student population as a large portion of their school's population. The administration was described by multiple teachers as very supportive of the LGBT student population, giving voice to the QSA, and creating student-led anti-bullying campaigns throughout the academic year. The overall sense at Bayview was that bullying was not tolerated. Members of the administration and teachers used the term "zero-tolerance" to describe the anti-bullying policy, and a link to the district policy was provided on the school's website. Due to the commitment to LGBT youth, bullying prevention, and the presences of a supportive administration, Bayview High School and Avenue High School were model schools for this study population. 
In contrast to Bayview High and Avenue High School, the student population at Central High, Dover High, and Eastpointe High Schools were described as low-income, with up to $70 \%$ of students on free or reduced-price lunch. The student body at each high school was described as very diverse, both ethnically and racially, in comparison to the larger city which is predominately white. At Central High School, there were some teachers who expressed a commitment to LGBT youth, with one teacher referring to the district's support of the LGBT youth population. However, there were reports of teachers not being supportive of LGBT youth. I cannot speak to the school administration's role in creating an environment conducive to protecting LGBT youth because the administration did not participate in the study. In terms of the anti-bullying policy, a link was provided on the school's website to the district anti-bullying policy.

At Dover High School, the policy was not available online, and it was evident through teacher discussions that the anti-bullying policy was not a topic of discussion in the school. There was some support for the LGBT population in that there was a studentled LGBT youth group but bullying was not a topic that came up frequently in the school. At Eastpointe, although the administration had a clear zero-tolerance approach to bullying, one teacher expressed skepticism in their belief that the school's administration would be supportive of LGBT youth, or handle incidents related to LGBT youth appropriately. The policy was not visible on the school's website, and teachers had limited resources for protecting LGBT youth from bullying or in general. One teacher did credit the school's racial and ethnic diversity as a reason why they had not heard any derogatory remarks against LGBT students in the school. The demographic characteristics of the schools, coupled with individual differences between teachers and 
administrators, are an important to understanding how schools addressed issues related to LGBT youth and bullying in general. I would expect given the city's commitment to the LGBTQ community, and the urban and diverse nature of the school district, that overall schools would be supportive of LGBT youth. Comparisons across schools will be made throughout the analysis to provide a comprehensive look at the relationship between school anti-bullying policy, implementation, and LGBT youth. Specifically, school differences will be described when examining participants' familiarity with the policy, experiences with training, perceptions of bullying as a problem, and approaches toward policy enforcements and bullying prevention.

Policy structure, addressed in Chapter IV, supports and expands the existing literature in illustrating the important role that policy context, and the school structure created by the policy has in bullying prevention, and policy implementation (Coburn et al. 2013). Chapter IV also draws attention to problem framing around bullying in schools, as well as role of training on the policy and specific training on the prevention of bullying based on perceived or actual sexual orientation (Coburn 2006; James et al. 2008; Greytak et al. 2013). Chapter V focuses on the factors that support or inhibit teachers from protecting LGBT youth from bullying. Chapter V illuminates the impact that limited social capital, based on district priorities and expectations around racial inequity in the schools, has on teacher resources and ultimately their ability to protect LGBT youth from bullying. 


\section{UNDERSTANDING ANTI-BULLYING POLICY: HOW POLICY STRUCTURE CREATES BARRIERS TO IMPLEMENTATION WITHIN SCHOOLS}

Although a policy outlines the rules and regulations for a school, how the policy is written can either help or inhibit schools from implementing the policy. A school policy that explicitly states bullying is prohibited based on sexual orientation or gender identity sends a message to administrators, teachers, staff, and students that bullying against LGBT youth is not welcome within their school (Kull et al. 2015). Past studies also indicate that policies that are inclusive of LGBTQ youth are essential for ensuring that LGBTQ youth feel safe, supported, and protected in their school environment (Hatzenbuehler et al. 2013). Anti-bullying policies play a vital role in structuring the school, creating clear expectations for behavior, guidelines for reporting, and outlining the consequences for bullying incidents. In addition to behavior, reporting, and consequences, school policies also frame a problem for teachers and administrators to address. Identification of this problem begins at the district level when the policy is created and passed on to schools (Coburn, Matia, and Choi 2013). In this chapter, I examine the role of anti-bullying policies in structuring schools, and argue that for the participants in this study, the presence of anti-bullying policy did not effectively translate into the practice of policy implementation, bullying prevention, or the protection of LGBT youth.

I argue that the policy structure, both in the language of the state law and district policy on bullying created barriers for schools to implement the anti-bullying policy. Specifically, the following factors were barriers to effectively enforcing anti-bullying policy in schools: 1) lack of teacher and administrator training, 2) teacher and 
administrator lack of knowledge of the school policy, 3) teachers' and administrators' lack of knowledge of bullying as a problem in their schools, and 4) reactive approaches to bullying prevention. This chapter primarily focuses on the anti-bullying policy in general, with examples related to LGBT youth. There is one section within this chapter on training specific to LGBT youth as well. Before discussing these factors, I include a brief description of the state, district, and school policies in the beginning of the chapter and conclude the chapter with an account of participants' perspectives on best practices for bullying prevention, as well as suggestions for improving their school's anti-bullying policy.

The Policy Context: State Law, District Policy, and School Policy

Before discussing the participant interviews, it is important to briefly describe the policy context for this study at the state, district, and school levels. In this particular state, the anti-bullying legislation is unique because the state is one of the few states in the U.S. that includes sexual orientation in its protected classes. Previous research indicates that enumeration of sexual orientation and gender identity aid in not only creating a supportive environment for LGBT youth, but also encourage youth and school staff to report incidents of bullying and harassment (Kull et al. 2015; Kosciw et al. 2014). In this section, I will discuss the state's legislation on bullying, the district's anti-bullying policy, and the school-level resources for understanding the district's policy (i.e. the student handbook). At each level I will describe the overall language of the policy, the inclusion of sexual orientation and/or gender identity, and expression. In addition, I provide a brief discussion of how the translation from state law, to district policy, to 
school resources created challenges for teachers in terms of their knowledge of the district policy.

At the state level, the anti-bullying law coupled bullying with harassment in its definition. Harassment and bullying were defined as acts that inhibited a student from having a supportive school environment. Bullying could include any harmful physical act toward a student but also could include causing the student psychological distress, or creating a hostile school environment. Sexual orientation was included as a protected class but a definition of sexual orientation was not provided. Gender identity/expression was not included among the protected classes and was not mentioned in the state legislation. The most notable requirements for districts are as follows: 1) create a policy prohibiting bullying and harassment, 2) define bullying and harassment, 3) create a protocol for reporting instances of bullying and harassment, 4) require employees and encourage students to report instances of bullying and harassment, 5) identify who is responsible for implementing the policy, and 6) make the policy accessible through the student handbook and school or school district websites, if available. It is important to also note that districts were encouraged to incorporate bullying prevention into their existing training programs for both students and employees. Districts were also encouraged to form task forces and create initiatives to prevent and respond to bullying. In terms of policy enforcement, the state mandate required districts to create a uniform procedure for reporting of incidents within the district. The procedure included naming a person responsible for investigating incidents, requiring school employees to report instances of harassment or bullying, and making sure that the policy clearly states the consequences for employees who do not report an incident. 
At the district level, there were two important documents pertaining to bullying: the district anti-harassment policy and the student handbook. Regarding the district antiharassment policy, harassment and bullying were defined using the same definitions provided in the state law. Sexual orientation was included as a protected class and defined as pertaining to a student's actual or perceived sexual orientation, or gender identity. One major difference between the state law and the district anti-harassment policy was the inclusion of gender identity. Gender identity was defined as an individual's identification as male or female, regardless of their sex assigned at birth, or their appearance/behavior. The inclusion of gender identity is an important piece toward recognizing how inclusive this school district is in terms of LGBT youth, relative to other school districts within the United States. Kull et al. (2015) found that only $10 \%$ of school districts in the United States, out of a sample of over 9,000 districts, included both sexual orientation and gender identity/expression in their district's policy. In this particular district, the inclusion of both protected classes would provide schools with support to create very supportive environments for transgender youth, as well as LGB youth.

In addition to the inclusion of gender identity in the district policy, the district policy also required schools to make sure that all students were aware of the definition of harassment, the consequences for harassing another student, and their responsibility to report any acts of harassment that they witness. Schools were also required to post antiharassment or bullying signs throughout the school. Regarding consequences, the district named the school principals or designated personnel as responsible for handling investigations of bullying. The principal or person designated to be responsible for investigating incidents was required to ensure that the incident was examined 
appropriately, and keep the identity of the participants confidential. Employees were also required to report any incidents that they were concerned about, and send their reports directly to a school official. Aside from notifying the parents once the incident concluded, no other procedures for the enforcement of the policy were included. Students found in violation of the policy would face disciplinary action ranging from a meeting up to an expulsion. The language within the district policy explicitly depicted bullying as a disciplinary issue, which reflected the language at the state level.

In terms of schools, the student handbook for the district served as an authoritative document for all schools in terms of bullying and harassment, based on participants' accounts of the handbook as a source of learning about the policy, a topic discussed further in this chapter. Review of the document revealed a focus on disciplinary action. There was an entire section on discipline in the student handbook. In the discipline section, a grid outlining possible offenses and their corresponding consequences were included. Definitions of harassment and bullying were included in this section. Harassment or bullying was defined as physical, verbal, or psychological, including abusive language. There were separate sections for harassment or bullying based on disability, race, and sex. For each possible offense, students could be written up for a conference, intervention, suspension, or expulsion. Sexual orientation and gender identity were not included in the list of possible offenses. The absence of sexual orientation and gender identity is the greatest distinction between the state, district, and school-level policies on bullying. If the school handbook does not identify perceived or actual sexual orientation or gender identity as possible forms of harassment or bullying 
that can occur at the school, bullying of LGBT youth may not be as salient of an issue for teachers and administrators.

At the school level, Avenue High School provided a link on the school's website to their school's handbook. Their school handbook was different from the district handbook but did include some elements of the district student handbook, including defining bullying/harassment as one category and listing the corresponding disciplinary actions that would result if a student were caught bullying another student. Students were also required to report an incident if bullying or harassment occurred. Bayview High and Central High included a link to the district website but the link did not direct the user to the student handbook, only the district department website. Dover High and Eastpointe High did not have their student handbook listed online, or provide links to the district website. Since the state law does require districts and schools to make the policy readily available, which included school websites (if available), not only does the lack of information demonstrate a deviation from the state law, but also illustrates that teachers, students, and staff at Dover High and Eastpointe High may not be knowledgeable of the school district's policy on bullying. Knowledge of the policy demonstrates an understanding that bullying is an issue, how to address bullying when it occurs, and helps both teachers and administrators to feel confident in their skills in terms of intervention and implementing the policy. As the following section demonstrates, all teachers and administrators were not knowledgeable of the district policy, or the student handbook.

\section{Understanding of the Anti-Bullying Policy: Familiarity, Awareness, and Exposure}


Review of the policy context at the state, district, and school level illustrates that bullying is conceptualized as a disciplinary issue, districts and schools are required to adopt anti-bullying policies, and the policy should be readily available to administrators, teachers and staff. However, assessments of participants' understanding of the policy, and their exposure to the policy reveal that the policy was not readily available to all teachers and administrators. As described by researchers interested in understanding how teachers build skills to implement school policies, being aware of a school's policy and being familiar with the explicit language are central to knowing how to appropriately respond if and when an incident does occur (Coburn and Russell 2008). Across all 21 interviews, familiarity and awareness of the anti-bullying policy varied significantly. When asked if they were familiar with the policy the majority of participants' knowledge of the policy ranged from very familiar to somewhat familiar. Among participants who were familiar with the policy, references were made to the student handbook, a zero-tolerance policy, and the ability to recite the specifics of the policy. In this section, I argue that the lack of familiarity and training on anti-bullying policy was a barrier to implementing the antibullying policy and protecting LGBT youth from bullying.

Within Avenue High there was a noteworthy degree of variation among administrators, teachers, and staff. Sarah and Mark, who are both administrators, varied in their conception of the policy. Mark was more familiar with the policy's terms and focused more on the school's efforts to respect differences. When describing the school's anti-bullying policy, Mark demonstrated a more in-depth knowledge of the protected classes: "So it's, well, students cannot discriminate, harass, or bully students on the basis of sexual, gender, sexual identity, gender identification [...]." Sarah, on the other hand, 
made more a reference to an individual's interpretation and perception of harassment, "it's how it's perceived by the victim [...] whatever they interpret it to be $[\ldots]$ Some folks get offended by innuendos a lot easier than others but ultimately it's their definition of what they see harassment is." For Sarah, her definition of harassment was not synonymous with the language in the district policy, which clearly defined harassment as any unwanted physical or verbal behavior that is harmful to a student. Comparatively, each of the administrators' responses demonstrated differed not only in how bullying and harassment as defined, but also how the policy as perceived by both administrators. For Mark, the bottom line was that students had a responsibility to one another to respect each other's differences, and not mistreat anyone, regardless of their identities. For Sarah, harassment was conceived as an individual issue, that varied based on someone's perception but also their tolerance for certain behaviors. As individuals responsible for the enforcement of the policy, both on the discipline side and in setting a tone for the rest of the staff and student body, their differences in perceiving the policy could have a significant effect on how teachers and students respond to bullying instances and/or perceive bullying as a problem. In terms of administrator knowledge of the protected classes, research demonstrates that students are more likely to report an incident if they are aware that their school's anti-bullying policy is inclusive of LGBT youth (Kull et al. 2015).

Following the examination of administrators' familiarity with the policy and the existing literature, it is possible that the differences in how administrators conceived of the policy affected how teachers both interpreted and experienced the policy themselves (Coburn 2001). For teachers and staff at Avenue High, responses reflected knowledge of 
the policy's location in the student handbook, the disciplinary aspect of the policy, and an honest assessment of their lack of knowledge of the policy. The availability of the handbook on their school's website could have contributed to fact that they at least were aware of the policy's location. Jane and Zachary, the school psychologist and a teacher at Avenue High, had a more comprehensive understanding of the policy, both explained that the policy protects all students, regardless of identity, that the policy was contained in the student handbook and that there were specific procedures for reporting and investigating incidents. In contrast, Zoe expressed her lack of awareness of the policy, drawing attention to a lack of training, "Only in the sense that we have a policy against it, there are some procedures in place [...] I honestly haven't read the policy in a long while or at all and we have not gotten specific staff training on the policy since I've been here I believe.” Given that Zoe was one of the school's QSA advisors, Zoe's reflection reiterates the importance of ensuring that there is a school-wide consensus not only on how bullying is understood, but also how the policy itself is conceptualized by teachers and staff members.

In contrast to Avenue High School, where administrators varied in their understanding of the policy itself, at Bayview High, there was a clear mention of zerotolerance in approaching bullying in the school, by both administrators and one teacher, which may be due to administrators' interpretation and subsequent messaging regarding the policy and bullying (Coburn 2004). Zero-tolerance refers to an approach to policy enforcement that involves focusing on the use of discipline to enact a policy. For bullying, zero-tolerance would mean that students receive some form of disciplinary action (i.e, referrals, expulsions, suspensions) when they are caught bullying. Both 
administrators made explicit references to not tolerating any kind of bullying in their school. Anthony, an administrator at Bayview High School, responded in a way that was the most aligned with the actual language of the state and district policy: "The policy is pretty much a zero-tolerance policy for any kind of behavior that is intimidating, harassing, or bullying, including we have a separate kind of section on social media, cyberbullying kinds of stuff." It became very evident throughout interviews with the majority of the teachers at Bayview also adopted the zero-tolerance model for addressing bullying in their school. Nathan's response best captures this consensus among teachers:

In my own words? [...] We take it pretty seriously here. We've had some incidences $[\ldots]$ So we've been doing a lot of things about that. The specific policy is that any bullying of any kind will not be tolerated and can result in immediate suspension and/or expulsion.

In terms of zero-tolerance, the approach taken by Bayview High aligned with the language of the district and state anti-bullying policy, which focused primarily on disciplinary actions for students who are caught bullying or harassing their peers. In the district handbook specifically, there were codes attached to every possible offense, ranging from conferences or an intervention (i.e., detention) to the maximum penalty, a one-year expulsion. Although zero-tolerance policies have come under extensive scrutiny in the past decade, existing students also suggest that zero-tolerance policies are useful for protecting LGBT youth because they send a message to the students that bullying against LGBT youth is not acceptable and has serious consequences (Misha et al. 2009; Stein 2003; Pollock 2006). At the very least, the efforts of Bayview High School were reflective of an attempt to create a normative environment where bullying against LGBT youth was not tolerated. 
The fact that the majority of participants who did express familiarity with the policy were employed at either Avenue High School or Bayview High School speaks to the commitment that both schools have toward bullying prevention, but also again the strength of their administrations and the overall commitment to LGBT youth at both schools. While there were a few teachers at Avenue High School and Bayview High School that expressed limited knowledge of the anti-bullying policy, most participants who were less familiar worked at Central High, Eastpointe High, and Dover High School. In contrast to participants who were more familiar, over a quarter of respondents interviewed were not familiar with the policy. Emily, a teacher at Bayview High, expressed her lack of knowledge of the policy but also suggested that information about the policy was not given out as readily as it should have been in her opinion. Emily's response relates back to the role of administrators and also suggests that Emily had not interacted as frequently with the school administrators, compared to other teachers at Bayview that expressed a clearer understanding of the policy, and its implications in terms of disciplinary action against students. The same can be said for Rose, a teacher at Avenue High, who discusses the lack of knowledge but also training regarding reporting protocols:

I would say that I know we have one and is something that there would be severe consequences if you got caught bullying, but I feel like I've never really had to use the policy myself and I haven't been, that I can remember, walked through, "this is what you do if someone is bullied." This is my first year here.

Rose's response demonstrates a critical problem with the current structure for providing teachers with information on the anti-bullying policy. Since Rose could not recall if she had ever been taught how to intervene if someone is being bullied, it is also likely that 
Rose was unaware which administrator to go in order to report a bullying incident.

Rose's response is echoed by teachers at Central High, Dover High, and Eastpointe High, demonstrating that some teachers were very limited in their ability to recall the explicit language in the policy. If teachers are not familiar with what the policy itself states, how can they be fully equipped to address an instance of bullying when it does occur? When considering levels of familiarity within schools it is worth noting that across all schools except Eastpointe High there were teachers who were not at all familiar with the policy. The lack of familiarity speaks to the importance of making sure that all teachers are familiar with the policy, and that the policy is readily available to everyone. The lack of knowledge of anti-bullying policy is a clear barrier to being able to effectively protect students from bullying. Teachers' knowledge of the policy is reflective of not only their individual responsibility to read the student handbook, but also the responsibility of the school administrators to ensure that teachers are knowledgeable of bullying protocol and the policy language.

In terms of exposure, when asked how they learned about their school's antibullying policy, several teachers discussed the handbook, new teacher orientation, district training, and professional development meetings. The most notable responses came from participants who previously expressed their lack of familiarity with the policy. Taylor, a teacher at Dover High, when asked about his familiarity with the policy, expressed that he could not recite the policy or recognize it across districts. Yet, he later admitted that he was aware that student handbook contained the policy. Taylor's response illustrates the level of individual accountability that falls on teachers to read the school handbook, but also suggests the need for increased accountability for the administrative staff to make 
sure that all teachers are aware that the expectation is that they would read the handbook themselves. It may also be the case that teachers are both overwhelmed with their responsibilities at the beginning of the year and have limited time to remember every policy they were read during professional development meetings. This was expressed by Rose, who teaches at Avenue High:

At the beginning of the year there's a lot of nuts and bolts, kind of meetings where teachers gather and we'll go over policies about like school-wide policies and I vaguely remember there being some presentations around "this is what we've been doing around bullying" but I would say it's more informal.

For Rose, although there was some exposure at the beginning of the year, it was informal, which may have resulted in Rose not taking the information as seriously had she been required to read the policy again during the school year. Rose's experience was expressed by other teachers across the district, who vaguely recalled being exposed to the policy but could not remember any specifics about the policy or its enforcement. Each example illustrates that both individual factors, through teacher availability/interest and institutional factors (i.e. meetings and administrator messaging) are essential to fostering awareness of the policy. These factors are also important for understanding what may motive teachers to implement their school's anti-bullying policy. Inconsistencies regarding where teachers learn about the policy creates additional barriers to policy implementation, as teachers may not recall their past exposures to the policy. The following section examines the impact of training on policy implementation.

\section{The Role of Training in Anti-Bullying Policy Implementation}

The most concrete example of how teachers' and administrator's exposure to the policy effected their ability to intervene in bullying incidents was best displayed through 
analyses of their experiences with training. The previous section demonstrated that not all participants were familiar with the language of the anti-bullying policy, and several participants expressed limited knowledge of the policy, and how to intervene in bullying when it does occur. At Avenue High School and Bayview High School, although teachers and administrators at both schools demonstrated greater knowledge and awareness of the policy, the schools differed in their conceptualization of the policy, with Bayview making explicit references to zero-tolerance, and teachers and administrators at Avenue High School recognizing the policy as a message to the students to respect everyone's differences. At Central High, Dover High, and Eastpointe, High, some teachers expressed little to no knowledge of the policy. The discrepancies in teacher awareness of the policy created significant barriers to protecting youth from bullying. Existing studies suggest that teacher and administrator training through professional development meetings, and school-wide trainings, have positive effects on teachers' confidence in their ability to intervene when bullying occurs, self-efficacy, overall empathy for LGBT youth, and reductions in youth victimization (James et al. 2008; Flygare, Gill, and Johansson 2013; Mishna et al. 2009; Greytak, Kosciw, and Boesen 2013).

In this section, I examine participants' accounts of training specific to the antibullying policy and pertaining to preventing bullying based on sexual orientation. Similar to participant accounts of their familiarity and exposure to the policy, there were dissimilarities among both teachers and administrators in terms of training. These dissimilarities provide additional support for my argument that the language of the policy created an environment where it was difficult for teachers and administrators to implement the anti-bullying policy. The structure of the policy created significant barriers 
to implementation. Although the state law stated that school districts must add prevention and intervention of harassment and bullying into their existing training programs, participants accounts demonstrate that few teachers were adequately trained on the antibullying policy. In the district anti-harassment policy, only students were identified as individuals that should be informed of the protocol for reporting and the definitions in the policy. The discrepancy between the state law and district policy on bullying were problematic. If administrators only read the district policy, they would not be compelled to create training programs or add bullying and harassment to their existing staff trainings.

In order address the role of training in protecting LGBT youth, participants were asked if they ever received training specific to the anti-bullying policy at their school, as well as their involvement in training or programs geared towards preventing bullying based on perceived sexual orientation. Remarkably, across all five schools, over half of the participants reported never having training specific to the anti-bullying policy. Avenue High School was the only school in the study where participants discussed a school-based training. Jane, the school psychologist at Avenue High School, talked about how the school had developed a training for the students and faculty that was administered each year. For Jane, creation and delivery of this training was a part of a larger prevention model for the school. Jane stated:

If you can make changes to make students feel safer at school through training, then you are going to save a lot of problems down the road, discipline problems, social and emotional problems the students might develop. It's more of a prevention model and we're very invested in that prevention model. 
The anti-bullying training program involved both students delivering presentations to other students, in addition to presentations to teachers and staff every year. However, the majority of teachers interviewed at Avenue High School had not been trained on the specific policy. Rose and Zoe had never received training on the anti-bullying policy, Zachary stated that there was a district training on all district polices for new teachers, and that at Avenue High School teachers were not only required to read the policy to their students, but also had to read and sign the policy themselves when they begin teaching at the school. Jane's comments illustrated an even greater disparity in teacher training, by stating that all staff, teachers, and students were trained every year on the school's antibullying policy.

The differences in receiving training that were evident between participants are important to highlight for the following reasons. First Rose, Zoe, and Zachary had all been at Avenue High for less than 5 years. Zoe, who admitted no knowledge of the policy and never having been trained, had been working at Avenue for the past 4 years, while Zachary had only been working at Avenue for 2 years. Moreover, Henry, who also taught at Avenue High, described how even though he was aware of a presentation on bullying, he did not go regularly. When describing the training, he stated, "I did every year for a few years and I think because it's principally the same presentation that now I don't think I did it this year [...] but certainly it's a part of every new teacher's [training] and it's referenced frequently." It was evident that even though the presentation and training had value, Henry did not feel the need to attend every year. The fact that trainings were available for new teachers but not for existing teachers placed a lot of the responsibility on individual teachers. If trainings are not required for every teacher and staff member in 
the school, there is no guarantee that teachers will attend, or gain the skills necessary to feel comfortable intervening when they witness bullying. These distinctions are even more significant given that there was not a consensus among the newer teachers at Avenue High School in terms of the existence or frequency of the school's training. Jane, who had been at Avenue High for over a decade, insisted that every teacher had been trained when that was clearly not the case, raising questions about how teachers find out about the school's training and if they were required to attend or if the training was optional.

Drawing comparisons between Avenue High and Bayview High School, it was also apparent that even though the school administrators demonstrated a commitment to the student body's awareness of bullying, teachers were not discussed when asked about training specific to the anti-bullying policy. None of the teachers interviewed received training specific to the anti-bullying policy. Nathan provides the most thorough reflection on the lack of training for teachers:

I think they have gone over it at a staff meeting. They have yearly, I think they have to tell us what it is. As staff members we are expected to read the handbook. Does everybody do that? No. Do they talk about it for at a staff meeting, do we have a full professional development about that? No.

Nathan's comment illustrates an important point in understanding teacher training. For one, Nathan points out the expectation to read the student handbook, again reiterating the level of individual responsibility that is required in order for teachers to be able to effectively implement their school's anti-bullying policy. Nathan suggests that not all teachers read the handbook, and that collectively there was no formal training for staff that occurs throughout the school year. Emily, who also teaches at Bayview, described 
her lack of training on bullying, "We've had a lot of equity training and we had a suicide prevention training, that kind of stuff, but I would not say we have had anything that is directed at bullying, no." Emily's mentioning of suicide preventing and equity are very important. Both suicide prevention and equity are required trainings for teachers, in addition to child abuse prevention. The lack of school-wide training at Bayview High and Avenue High had an effect on the ability of teachers and administrators to intervene when witnessing bullying, let alone develop prevention strategies (James et al. 2008).

The issue of optional trainings only explicitly came up at Central High School. Caleb, who teaches at Central High, described his frustration with optional trainings: "we all complained that we don't really have any training around it and all the trainings are optional so they offer it but we have to take our professional leave time to do that." Caleb's comment was very telling of the not only the enthusiasm and dedication of the teachers at Central High, but also the lack of structural support from their school in terms of ensuring that teachers were able to build the skills they needed in order to protect students and be informed about school policy.

Across occupations, the majority of administrators reported training specific to the policy, most commonly conducted through the school district. This reveals an important distinction between the role of teachers versus administrators not only in the school but in the district as well. Even among administrators, one administrator may be more knowledgeable of the school's policy, based on differences in terms of the content of their trainings at the district level. Brenda, an administrator at Bayview High School, described a difference between training for principals versus vice principals. She stated that vice principals were made aware of the district policies and disciplinary matters. The 
distinction between principals and vice principals is an important piece to understanding how training may impact policy implementation, and reinforce the use of reactive policies. If vice principals mostly handle disciplinary matters, it would make sense for the school to have a reactive approach to bullying. Unfortunately, additional accounts from participants revealed that this is not the case. Anthony, the other administrator at Bayview High, recalled having some training specific for administrators during a leadership training at the district level. Anthony was very knowledgeable on the specific language of the anti-bullying policy, and did have training on the policy itself. Brenda, on the other hand, discussed never having training specific to the anti-bullying policy, "we're going through a training process now in which we meet once a month and we are reviewing the different policies but there hasn't been a training that is solely for antibullying." Brenda's previous discussion on vice principals being trained on the district policies contrasts with her admitting that she had never been trained on the anti-bullying policy.

At Avenue High, both administrators reported receiving training. Mark had a more vivid recollection of the training he recently received, he stated,

$[\ldots]$ there's a review training that kind of happens and then we had just an antidiscrimination, harassment, and bullying training for I think it was 4 hours last summer. So I have had all of those and some of them of course are sexual harassment and racial microaggressions and harassment.

Mark later went on to discuss how some of those trainings included information on sexual identity, which Sarah described as pertaining to what language LGBT youth use to describe themselves. Once again, the differences between administrators at Bayview High School and administrators at Avenue High School demonstrate that each school 
held different beliefs in terms of the approach to bullying. Both Bayview administrators made references to the disciplinary aspect of training, while the protected classes and perceptions of individual students were salient for Mark and Sarah. As a whole, examining participant accounts of trainings specific to the policy reveal dissimilarities across schools, and occupations. The differences across teachers created challenges when it came down to understanding how to intervene, or who the policy protected. If teachers are not knowledgeable of the protocol for intervention, they may not step in when students are bullied. The dissimilarities between administrators was problematic because they were responsible for investigating incidents, and also because they set the tone for how the teachers perceive the school's policy (Coburn 2006). This also extends to training specific for LGBT youth, the topic of the next section.

\section{Training Specific to LGBT Youth}

Devoting both time and resources toward ensuring that all school personnel are well-versed on the issues that affect LGBT youth, as well as how to address bullying against LGBT youth, are essential to providing a supportive school environment (Hatzenbuehler and Keyes 2013). With proper training, teachers and administrators can feel confident in their abilities to intervene with LGBT youth are bullied, and studies indicate that administrators and teachers report increased self-efficacy, as well as empathy towards LGBT youth as a result of trainings (Greytak et al. 2013). When it came to protecting LGBT youth, all participants agreed that the policy can and/or should be used to protect against harassment based on perceived sexual orientation. However, examining their responses to questions of training specific to LGBT youth revealed that 
they lacked adequate training to achieve this task. Among participants, over half reported that there had not been trainings or programs for bullying prevention for LGBT youth in the past year, and roughly a third said that in the past year there had been either a training or program for staff geared toward bullying prevention for LGBT youth. Among those who reported not having training, participants recalled a focus on LGBTQ issues or awareness of students' sexual orientation. Emily, who teaches at Bayview, describes the limited about of information she had received about LGBT youth: "there has been very minor training around transgender awareness stuff. There has been a little bit and LGBTQ awareness [...] I guess I wouldn't say those are necessarily trainings as much as it's a part of our professional development where they say and give some stuff out about it." Although Emily was exposed to LGBT issues through professional development, it was very clear that specific training on the prevention of bullying based on perceived or actual sexual orientation and/or gender identity was not a requirement for the teachers and staff.

In contrast to Emily, Nathan and Daniel, who also teach at Bayview, did report receiving training on bullying prevention specific to LGBT youth. When asked about the training, Nathan replied by stating, "working with LGBTQ students [...] and some training in that regard too. They really have addressed specifically, sexual identity and gender identity as well as really focusing on race." Daniel expands on Nathan's comments and explains in more detail some of the skill-building activities that are conducted in the trainings:

Again it's something really pervasive in everything we do. I remember the [...] the quick quiz thing where you associate good and bad with different images and different people, and we took it for sexual identity, for age, for race, for class, just 
to see where we are as humans and then adapt our teachings around where our holes are or where our blind spots are. And we often times have books passed out to us to read and to continue building our knowledge base.

Daniel described a very comprehensive training that involved challenging everyone to confront their own biases. Daniel stated that the training was delivered by the administration, who often collaborated with the district to deliver these trainings. It is evident that Daniel and Nathan had very different experiences as far as receiving training in the school on LGBT youth and bullying. Similar to Bayview, teachers at Avenue High had very different experiences in terms of bullying against LGBT youth. Zachary, Rose, and Zoe all reported that there had not been any trainings in the past year geared toward preventing bullying based on perceived sexual orientation. Both Rose and Zachary mentioned the district-wide equity training that was required of staff. Zachary assumed that the equity training would have information on prevention of LGBT-based bullying but admitted that he did not attend that training. Zoe recalled a required video from in the past year, "the only thing we did was watch a video that was made with students in the district last year speaking about their experience as LGBTQ students. That was a required video for all staff to watch this year." Zoe, Zachary, and Rose demonstrate that even among participants who did not receive training, teachers have very different experiences and are not all on the same page when it comes to training.

For one teacher at Avenue High School, the fact that the LGBT youth population had gained a lot of visibility, and was very supported throughout the school, meant that LGBT youth and bullying did not need to be a primary concern within the school. Henry, who stated that preventing bullying based on sexual orientation had been an integral part of the school's approach over the years, stated that there was not much of a need to make 
LGBT youth the primary concern, referencing the success of the QSA, "I think race and socioeconomic status are bigger issues here [...] it's been pretty well addressed. I think if you went to a QSA meeting the room's packed [.... it's a very robust group of kids." Considering the previous literature on the benefits and drawbacks of focusing on GSAs, in addition to the evident inconsistencies at Avenue High, in terms of teacher awareness of LGBT bullying, training on the anti-bullying policy, and training specific to LGBT youth, Henry's comment again reflects the variation in teacher attitudes towards bullying, and the evident need for a school-wide approach to teacher training.

In contrast to the teachers at Avenue, both Administrators, Mark and Sarah, did report having training specific to bullying prevention for LGBT youth. Both Mark and Sarah reported a required training every year, revealing differences between administrator and teacher trainings within the district. According to Sarah, every teacher gets also gets trained in the district each year. When asked to describe any differences between teacher and administrator trainings, Sarah replied by stating that administrator trainings tend to center around the laws and addressing incidents, whereas teacher trainings are focused more on supporting students. In describing specific elements of training for supporting LGBT youth, Sarah stated, "how to support kids and having a gender neutral zone, a safe zone that they call it. I know some classrooms that have the rainbow sticker on the front of the classrooms like this is a safe zone." This distinction is very key again because it sends an explicit message that the role of the administration is primarily disciplinary, whereas for teachers is more of a student support role. The pitfall of making these exclusions, when it comes to implementing their school's anti-bullying policy, is that teachers are not as aware as administrators are in terms of the actual policy 
and protocols themselves. As evidenced by participant responses, one consequence of this separation between administration and staff is that teachers are left to make critical decisions about if and how to intervene when bullying does occur in their school.

Considering the existing literature on the role of teacher training in protecting LGBT youth from bullying, the inconsistencies, and considerable lack of training on bullying prevention for LGBT youth provide support for increasing teacher training and mandatory training for all teachers, administrators, and staff within the school district to ensure that everyone is aware of what issues LGBT youth are facing in school. In the following section, I address the role of problem framing in setting up bullying as an issue in the schools. Participants' accounts of training demonstrated that at the school and district level, there were evident gaps in the provision of training on the anti-bullying policy, and specific training to address the prevention of bullying against LGBT youth. In addition to the lack of teacher and administrator training on the anti-bullying policy, there were also inconsistencies in terms of who received training and who did not. As a whole, the variability in teacher and administrator training is reflective not only of the lack of mandatory training on the anti-bullying policy itself, but also the lack of encouragement by both the district and the school administrators in terms of making sure that teachers and administrators are aware of trainings, and encouraged to attend trainings. The efforts of district and school administrators to ensure that teachers receive proper information and training on the policy plays a large role in how the problem of bullying gets framed for teachers, ultimately impacted their decisions to implement the school's anti-bullying policy (Coburn 2004). 


\section{Perceptions of Bullying as a Problem}

Similar to the issue of training, understanding bullying as a problem is an important aspect of understanding the anti-bullying policy, choosing to implement the policy, and being aware of the protocols associated with reporting bullying in one's school. Both the anti-bullying policy, knowledge of the policy, and training help to inform administrators and staff that bullying is a problem. When policy awareness and training varies, there can be differing opinions on whether or not bullying is in fact an issue in the school. District and school administrators play a vital role in articulating to other administrators and teachers that the anti-bullying policy is important, it aligns with the values of the district/school, and that trainings are an integral part of preventing and reducing bullying within the district/school. Both district and school administrators influence if and how bullying is conceptualized as a problem. Previous research indicates that teachers are influenced by the messages they receive regarding how or what to teach (Coburn 2004). Professional development meetings, policy documents, and contact with administrators or district personnel all serve as ways that teachers can receive messages about school reforms (Coburn 2004). School principals play an important role in problem framing, influencing teachers' understanding of a problem, and gaining support from teachers on the new policy (Coburn 2006). Coburn (2006) found that over time, teachers began to adopt the viewpoint of their school's principal, using the principal's messaging regarding a reading problem to make sense of the new reading reforms in their school (Coburn 2006).

When applying problem framing to bullying, an anti-bullying policy initially frames bullying as a school problem, creates opportunities for teachers and administrators 
to develop networks around the policy, and provides information on how to address bullying. Through repeated exposure to the policy, and conversations with other teachers and administrators, bullying is framed as an issue in the school (Coburn 2006; Coburn 2004). In the current study, I argue that participant interviews demonstrate that problem framing did not occur consistently throughout the district. While the district did provide a policy, and schools did choose to adopt anti-bullying policies, bullying itself was not uniformly framed as an issue, within or across schools. As expressed in the participant responses that follow, it is apparent that administrators believed that bullying was a problem broadly, but their beliefs about bullying did not translate into a school-wide belief that bullying was an issue in their school. As a result, teachers illustrated variation in their conceptualization of bullying as a problem in their school, as well as their understanding of whether or not bullying was an issue in general, or specific to LGBT youth, racial minorities, or sex and gender.

Participant accounts of bullying as a problem in the school are based on their responses to the following question: In your opinion, is bullying a problem in your school? Roughly half of the participants believed that bullying was a problem in general, without any mention to a specific protected class. For these participants, bullying was either understood as a broad issue (i.e. "bullying is a problem everywhere") or as an issue in the school that was not specific to any particular group of students. A third of participants did not believe that bullying was a problem in their school. The remaining twenty percent of participants referenced race, sex and gender, as well as cyberbullying. Only one teacher mentioned that bullying was a problem for LGBT youth when asked if they believed that bullying was a problem in their school. In his discussions, Caleb, who 
teaches at Central High School, made references to LGBT youth disclosing information about being discriminated against in their classrooms, without proper intervention from teachers. Among other participants, there was considerable variability within schools in terms of perceiving bullying as problem, as evident in Avenue High, where some teachers did say bullying was a problem, and some did not. For participants who did not believe bullying was a problem, general remarks included stating that they personally had not witnessed any acts of bullying in the school, that bullying was not something that came up daily for administrators, or that bullying had declined over the years at their school. According to Mark, one of the school's administrators, bullying was a problem everywhere, but he also noted that in their school bullying rates were declining and the school was supportive for transgender students in particular. This type of broad discussion of bullying as a general issue but not one experienced in the school was characteristic of others at Avenue High School. As expressed by one teacher, Zachary: 'kids don't say 'that's gay,' which is great. This doesn't mean students are not receiving those comments, because I'm sure they are, but it's a lot less." It is interesting that even though Zachary admits that he cannot rule out the occurrence of homophobic remarks, the prevalence of hearing those remarks has gone down. His phrasing is synonymous to the language that Mark used to describe bullying in the school, demonstrating that the administration has an effect on how teachers perceive problems in their school.

In this study, administrators helped to frame bullying as a general problem, but not necessarily an issue in their school. At Avenue High School, bullying was framed as a general problem, with notable declines in bullying at the school. The administration spoke of bullying as a problem in general, with no mention to specific groups of students 
that were being targeted. The administration's view on bullying at their school trickled down to teachers' perceptions and responses to bullying. When asked to recall any instances of bullying in the past year that stood out to him, Mark replied by discussing a recent issue between senior and freshman cohorts at a school assembly where upperclassmen were heckling the underclassmen, but this event was characterized as a microaggression. Sarah, the other administrator at the school, did agree with Mark that bullying was decreasing, and stated that bullying used to be a problem in the school. When asked about specific incidences, Sarah recalled a cyberbullying incident that occurred a week prior to the interview. Mark, on the other hand, admitted to being less familiar with specific cases because he does not handle most of the disciplinary issues in the school. This difference between administrators' roles affected their knowledge of specific bullying instance, which could ultimately affect how bullying was being framed for teachers. An additional element of being able to frame bullying as a problem is the interactions that teachers have with their administration (Coburn 2004). If one teacher interacts more often with Mark, they may not conceptualize bullying the same way as a teacher who interacts with and has more conversations about bullying with Sarah. Referring back to the teachers at Avenue High, Zachary, in contrast to the other teachers at Avenue High who did not recall specific instances of bullying, described a common form of bullying in the school that was based on race: "I see lots of students say like, 'you're Asian you should be killing this class!' [...] but in terms of physical and kids pushing each other, no." Comparing Zachary's initial comment about bullying as a problem in his school to his comment about race, it becomes apparent that bullying was being framed as general problem in the school, and the focus on was the success of 
reducing homophobic slurs. Moreover, for Zachary specifically, bullying may be characterized as physical in nature, which would explain why he considered the racial comment to be a microaggression. Nevertheless, the district policy and the student handbook did distinguish between physical and verbal forms of bullying and harassment. The participants' comments on bullying as a problem echo the increased national attention on bullying, by classifying bullying as something that happens everywhere, even if they do not see it themselves.

In addition to demonstrating how the problem of bullying is framed in the school, participant responses also illustrated the barriers associated with trying to prevent bullying and being aware of bullying in general. One teacher at Avenue High School did recall hearing homophobic words in the hallway. For Zoe, bullying was something they were confronting and witnessing in the hallways, but was not an issue and was not visible, either in their classroom. Zoe describes her experience hearing homophobic words in the hallway below.

the usual four letter words are rampant in the hallway [...] Every so often I will hear students using a homophobic word [...] and stop the student and have them explain to me what they just said and how it's not appropriate. Often I don't know the student so it's hard to have a one on one conversation. Because we don't have that relationship there.

When asked why she did not hear or witness bullying in her classroom Zoe responded by saying it was because it was happening outside of the classroom, school, or was happening online. Her response illustrates a challenge expressed by many teachers, who were limited in their ability to catch and intervene in instances of bullying due in part to the presence of cyberbullying, a challenge I address further in the Chapter V. 
Despite differences among teacher's perceptions of bullying as a problem in the school, Mark, an administrator, notes that the school first gained momentum in terms of bullying prevention when a few teachers decided that bullying was a problem in their school. Mark states:

one faculty member said "come on this really a problem here" and I distinctly remember eight to ten other faculty members just standing up and sharing for the student and saying "it's a problem here." And then that was it and from then on everyone kind of accepted that we need to do this.

Mark's comments regarding the role of teachers, staff, and students in petitioning for increased support around bullying illustrated that there were unique characteristics within Avenue High School that encouraged them to consider bullying as an important issue in the school. Jane, the school psychologist, was mentioned by both teachers and administrators as being instrumental in bringing awareness to bullying, and the prevention of bullying against LGBT youth. In particular, Jane was credited by multiple teachers and both administrators for her work in leading students in their development of the anti-bullying campaign and student-led trainings that were implemented every year at Avenue High School. Similar to both Mark and Sarah, Jane stated that bullying used to be an issue but was declining. Jane stated, "yes, we have the data to say that there was a problem and we have the data to say that we have made some significant gains in pretty much all areas." Jane referred to discipline report data when describing the declines in anti-gay, race, and sexual harassment reports in the past few years. Although Jane's comments do provide support for declines in bullying, her perception that bullying was not a problem is still problematic, as discipline data only captures the students who actually report bullying instances. 
When considered as a whole, the responses of participants at Avenue High School demonstrated the degree of variation in teachers' experiences hearing and witnessing bullying, as some teachers reported hearing phrases such as "that's so gay" were limited or did not occur at all while one teacher recalls an instance where they intervened after hearing a student make a homophobic remark. Their responses also illustrated that if bullying is not framed as a specific problem in the school, teachers will not view it as an issue. This was evident through previous accounts from both administrators, who viewed bullying as a general problem that occurs across all schools but not a specific concern at Avenue High School.

Similar to Avenue High, administrators at Bayview High School also believed that bullying was an issue broadly in schools but believed that bullying was declining in their school. Anthony attributed the school's efforts to declines in bullying, and recalled bullying instances pertaining to racism, sexual harassment, and special education students in the past year. Brenda, the other administrator at Bayview, stated that she had not seen much bullying reach the administrator level. While it is possible that administrators delegated responsibilities in terms of handling bullying instances, the fact that Brenda was not aware of any of the instances that had reached the administrative level demonstrates a discrepancy in administrator awareness of bullying issues in the school. The dissimilarities between Brenda and Anthony have the potential to affect how teachers perceive bullying in the school, but also illustrate that administrators may not communicate with one another about instances that do arise. These distinctions are important to understanding how each school is able to implement its anti-bullying policy, as the literature highlights the importance of social networks and communication among 
teachers and administrators in building competency around new school policies, and framing problems within the school (Coburn, Matia, and Choi 2013). The similarities between Bayview High and Avenue High School were reflective of their similar demographic characteristics, in addition to the similarities in the presence of a supportive administration, well-supported QSAs, and overall commitment to LGBT youth.

Among teachers at Bayview High School, there was specific mentioning of instances either in their classrooms or school-wide incidents. Issues pertaining to race, sex, and transgender identity were the most frequently mentioned types of bullying. Teacher accounts ranged from descriptions of instances in their classrooms that resulted in a meeting with parents, to sexual harassment cases leading to student's transferring. Nathan, a teacher at Bayview, described one incident involving race: "have I had kids report to me that they are being bullied? Absolutely. [...] I know there was a big instance about jokes, comments, bullying comments [...] targeted more specific to African Americans." In discussing this incident, Nathan also talked about how they have a zerotolerance policy in their classroom, don't see everything that happens, and isn't always given details about school-wide instances. Their reflections point out not only how bullying is prevalent in their school, but also that they are very knowledgeable that instances do occur, try to step in when possible, and recognize that they are limited in in their ability to be aware of every instance as a teacher.

When considered in connection to their understanding of bullying as an issue in their school, teacher accounts of bullying not being a problem were in contrast with their reflections on past instances. In contrast to Bayview and Avenue High Schools, teachers and Central High School demonstrate significant issues around physical violence and 
LGBT youth discrimination in the classroom. One teacher, Ryan, stated that they did not think bullying was a problem at their school but then immediately recalled an instance where one of their students was going to drop out of school because of bullying. Grace, who also teaches at Central High, described an instance where she and another teacher intervened in a potential fight:

I had to interrupt something in the hall recently, where a kid was following the other kid, like let's take it outside type of thing, and the other kid was clearly saying, "uh let's not." So another staff member and I intervened, we thought it was done but it fired back up and it became a larger deal with administration and I typed a thing up about that.

Grace mentioned more than one occasion where she either witnessed or was made aware of a students' involvement in physical fights, being threatened, or worried about a physical fight at school. Michael also discussed the frequency of fighting at the school, stating, "I felt as though the way that people who deal with fights all the time get calloused about it, that it's just like oh this girl's bad, this is the bad girl, this is the good girl kind of thing." Michael hones in on an important aspect of how bullying was experienced at Central High School. The sheer volume of physical fights normalized the issue, and created a situation where teachers were frequently stepping in but the overall issues were not getting resolved. Because the administration did not participate in this study, it is unknown to what extent the administration provided support to teachers in terms of understanding bullying and how the administration framed bullying as a problem in their school.

In terms of recognizing bullying as an issue for LGBT youth specifically, Caleb, who teaches at Central High School, was the only participant who stated that LBGT youth bullying was a problem. Caleb described bullying of LGBT youth as an issue in his 
school, discussing hearing the word "faggot" in the hallway. When describing an instance brought to his attention by students he expressed his frustration with other teachers: "I have a lot of gay students in my [...] class and they are constantly called the F-word and have a really hard time because it happens in class and nothing is done about it [...] and they think the teacher hears it." Comparing Caleb's experience to Ryan's, who later stated that they were not sure if the school had an LGBT organization and had never heard of anyone being bullied due to their sexual orientation, it was evident that there was not a clear message across the school that bullying was an issue, especially bullying based on sexual orientation or gender identity. Caleb later recalled another incident that lead a transgender student to transfer:

of the reasons they kept complaining about is that they had recently transitioned and goes by he pronouns and by a different name and one student they've known since elementary school refuses and talks about how they are still a girl and won't call them by their name and just like brings it up in class when it's unnecessary $[\ldots]$ and the teacher doesn't really do anything so they felt really attacked and they transferred.

The key point of this issue described by Caleb is not only the intolerance on the side of the student refusing to respect that their friend had transitioned, but also the lack of intervention by the teacher. Again, this incident reiterates the importance of framing bullying as a problem, training, and also making sure that teachers are aware of the specific types of bullying that can occur in the school and are included in the school's anti-bullying policy.

At Eastpointe High School and Dover High School, although there were discrepancies in teacher and administrator accounts of bullying, both schools seemed to have very supportive environments for transgender students, as evidenced by their 
discussions of past instances of bullying. George, a teacher at Eastpointe High, described when one his students wanted to use a different gender pronoun: "and it was actually in one of my more rowdy, rambunctious classes and there wasn't anyone who was like, 'hem why are you calling him he?'[...] I don't get the impression that they are really struggling." These positive experience described by George may reflect the stance of the administration at Eastpointe High. Molly, an administrator at Eastpointe High, had a very explicit zero-tolerance approach to bullying, and found any form of bullying to be unacceptable, which seemed to translate to the teachers and students at the school.

When asked specifically about instances of bullying based on perceived sexual orientation, Molly responded by saying that she did not believe that the student body at Eastpointe High targeted students who were transgender or LGB. Similar to administrators at Avenue High and Bayview High that did not believe bullying occurred often in their school, although Molly personally did not tolerate bullying, she did not perceive bullying of LGBT youth to be an issue in school. Molly's perspective demonstrates that administrators' beliefs regarding bullying are an integral part of their approach to bullying prevention, and ultimately how school policies are implemented. As a whole, participant perspectives on bullying as an issue revealed significant disparities in teacher and administrator understandings of bullying in their school. For administrators at Avenue High School, bullying was conceptualized as a general issue in all schools but an issue that was declining in their school. This perspective trickled down to the teachers at Avenue High school, who for the most part did not consider bullying to be an issue. Across the other high schools, teacher accounts varied in their understanding of bullying as an issue, with most teachers referring to general bullying, and a few referring to sex 
and race as issues. Only one teacher referenced LGBT youth, which highlights not only the differences in problem framing across the schools, but also differences in teachers' awareness of LGBT youth bullying as an issue. The perspectives of administrators at Bayview High and Eastpointe High, who viewed the policy as a zero-tolerance policy on bullying, were in line with the language at both the state and district levels regarding the anti-bullying policy and reinforce the impact that the policy language can have on framing an issue for the school administrators. Taken as a whole, the responses of teachers and administrators reveal school differences in terms of understanding bullying, an overall lack of awareness of LGBT youth issues that pertain to bullying, and differences how the problem of bullying was framed within the district. All of these factors have an effect on how teachers and administrators view enforcement of the antibullying policy, the focus of the following section.

\section{How is the Policy Enforced?}

According to the state law and district policy, school administrators, including the principal or other designated school official, were responsible for receiving and handling instances of bullying in their school. This is the first step in implementing the school's anti-bullying policy. Ensuring that all teachers know who to report to is an important factor in determining whether or not teachers will actually report any acts of bullying that they witness. The district policy also required school employees to report any witnessed acts of harassment against a student, in addition to reporting any knowledge that they have of a student being harassed in school. Although the state law and the district policy were clear on teachers' responsibility for reporting incidents, and who they should go to, 
examining teachers' and administrators', the language in either document is not clear on how to investigate incidents, and how incidents are actually handled. Thus, the details of policy enforcement were left out of the policy language, leaving the discretion in the hands of school administrators and teachers. In this section, I demonstrate that the lack of explicit guidelines on how to enforce the anti-bullying policy posed challenges particularly for teachers, some of whom were unware of when to intervene and who to go to. For those teachers who were familiar with the procedures for reporting, clear divisions between their role and the role of administrations revealed that they viewed their responsibility as teachers as merely a verbal recognition of the problem, passing the report up to administration, and trusting that the incident was appropriately handled or resolved.

In addition to discussing participants' accounts of how the policy was enforced, in this section I also evaluate how incidents were handled in the schools. Their responses illustrated the relationship between how incidents were handled, and the participants understanding of policy enforcement. I argue that their responses reflect primarily reactive approaches to enforcement, essentially demonstrating that implementing the school's anti-bullying policy involved simply reacting to incidents when they did arise. This approach was consistent with the language at both the state and district levels. Since bullying was framed as a disciplinary issue, the employment of reactive approaches aligned with the overall message of the district policy. In the student handbook, although school employees were encouraged to seek out strategies to reinforce positive behaviors among students they were also encouraged intervene as soon as possible and begin the process of disciplining students who misbehave. The district policy itself was never 
explicitly mentioned in the student handbook, and there was a long list of possible offenses for students. The list of offenses that did include harassment or bullying, as expressed earlier in this chapter, varied in the scope of their definitions. Given that there was no explicit mentioning of sexual orientation or gender expression, it is reasonable to assume that teachers would not feel confident intervening in bullying of LGBT youth, or be familiar with appropriate protocols regarding LGBT youth. All of these factors are an important aspect of the structure that each teacher and administrator had to rely on when it came to bullying in their school.

Regarding policy implementation, participants were asked about how the policy was enforced at their school and what their role was in its implementation. Their responses revealed that knowledge of how to enforce the policy affected not only teachers' confidence in enforcement but also what they would do if an incident arose. Among teachers, there was a notable amount of confusion and dissimilarity in terms of understanding how the policy was enforced at their school, as well as their role in enforcement. Two major approaches were employed by the schools in this study: zerotolerance and restorative justice approaches to policy implementation. The zero-tolerance approach refers to the use of disciplinary action to respond to incidents. For example, if a teacher were to witness an act of bullying, they would write the student up, giving the incident a numerical designation in their reporting system. The numerical designation corresponded to the level of disciplinary action required, ranging from a conference to an expulsion. Daniel, who teaches at Bayview, described the disciplinary protocol used when incidents do occur: 
Referrals are written right away. Our system is set up that we have those kinds of bullying codes for level 1,2, and 3 and when we hear it we drop a referral in and administration gets involved. And it notates when we call the parents, when we have conferences, what happens next, counselor's involvement.

Daniel's comment reflected not only the zero-tolerance language of the district policy, but also the no tolerance approach previously described by teachers and administrators at Bayview. Daniel's comment was reflective of the language at both the state and district levels that teachers' roles were to channel incidents up toward the administration. Daniel found this role to be very limiting, and expressed his frustration with having a limited role in policy implementation. When asked about his role in policy implementation, Daniel, expressed that his position as a first-year teacher meant he could not be involved in implementation at his school, despite expressing an interest. For Daniel, even though he was aware that there was a hierarchy in terms of reporting and responsibility, he personally believed that teachers should play a more integral part in how the school's anti-bullying policy was implemented. The clear designation of administrators as responsible for the discipline side of bullying was very evident at Bayview High School. Not every teacher held the same belief as Daniel, however. For Daniel, teachers were the primarily force behind enforcement, while for Nathan enforcement as primarily administrative. Nathan discussed the role of administrators further, highlighting challenges that teachers face when trying to see incidents through:

That's a little different because it happens so much at the administration level so it's difficult for teachers to really see it through. So if we were to report something that came to us or a student were to report something, it would go to admin. Administration generally deals with that.

Nathan's response illustrated the lack of consistent messaging around reporting and protocols for investigating incidents. Teachers were left out in terms of understanding or 
being aware of the result of incidents. Emily, who also teaches at Bayview, described an incident where the teachers were unaware if the incident was ever resolved. She described the administration's strategy as a "behind closed doors" approach. The comments provided by teachers at Bayview demonstrate how the state law and district policy created a system where teachers were not intricately a part of seeing incidents through to completion, serving as the initial point of reference due to their interactions with students, but ultimately being left in the dark in terms of how the policy was implemented in its entirety.

Despite explicit language in the policy regarding administrators' role in enforcement, teachers at Eastpointe High School and Avenue High School expressed a substantial amount of confusion around who to approach and teachers' role in enforcement. Eric, a teacher at Eastpointe High stated, "it would go that teachers and students are responsible and then our supervisors are as well [...] I would go to an admin if I had an issue but it's a guess which one I would go to specifically.” Although Eric was very aware of the hierarchy in their school, they were unsure which administrator to go to in the event of an incident. Eric's response further supports Coburn (2006) who suggests that it benefits teachers to interact with school administrators, who help to frame the problem but also encourage teachers to be actively involved in policy implementation. At Avenue High School, one teacher had no idea how the school's policy was enforced, while others mentioned student-led programs and school-wide prevention programs. Examples of student-led programs at Avenue High School included anti-bullying campaigns and the use of student-led presentations to train students on bullying prevention in the school. Zachary described the role of students in enforcement, "the 
biggest thing is that a lot of peers are stepping in, or upperclassmen saying, 'hey, that's not cool', write it up and there would be a meeting, a punishment, stuff like that." Zachary discussed how their students were taught to confront perpetrators of bullying. When asked if there were any instances in that last year that stood out, Zachary replied, "we really empower our students to speak up $[\ldots]$ look them in the eye and say "that was really shitty,' [...] because maybe the bullying can be like, 'whoa, okay, I was trying to be cool in front of my friends'." Although Zachary's comment positively reflects the student-led approach taken at Avenue High, the method described by Zachary is very reactive, and does not prevent an incident from happening. The acknowledgment of students playing an active role at Avenue High School is also a reflection of the administrative support for the policy and bullying prevention at the school, as evidenced by Mark's focus on student programs and school-wide efforts to address bullying.

Student-led programs was also an important aspect of Bayview High School's approach to bullying. Nathan, who teaches at Bayview, also described the role that students had in interrupting bullying when it did occur:

My guess would be that the majority of the bullying issues are dealt with by kids going directly to admin or coming to us and asking if it can get turned over to admin. Usually that first line of defense is coming from the kids. So one of these goals with the campaigns is educating them on what the policy is [...] for kids to call it out because they're the ones that see it.

As Nathan discussed, educating students on how to intervene when bullying does happen was an important part of the programs in their school. Similar to Avenue High School, the incorporation of student-led programs seemed indicative of the commitment of the administration at both schools to allowing students to be involved in addressing issues within their schools. 
In contrast to teachers, administrators expressed greater clarity in terms of the protocol for policy enforcement and reporting incidents. The fact that administrators were more knowledgeable of school protocols for reporting is consistent with the identification of administrators with enforcement in the policy documents. References to the discipline side of enforcement aligned with the messaging in the state and district level policies which clearly labeled bullying and harassment as disciplinary issues. Molly, an administrator at Eastpointe High, described her role in enforcing the policy

If I hear about it, it's enforced to the maximum penalty. If someone is found to be bullying or harassing someone else, if it's been ongoing and substantiated and intense they get the maximum consequence $[\ldots]$ I maximize it $[\ldots]$ and let them know we don't tolerate it and it's not a joke, and it's not taken lightly.

The fact that Molly was very aware of her role in providing discipline and Eric, who teaches at Eastpointe High is unsure of the protocol, let alone who he would report an incident too, reveals a very problematic school structure that hindered teachers from effectively reporting incidents and being actively involved in how their school's antibullying policy was enforced. Molly's comment was very indicative of the zero-tolerance language expressed in the anti-bullying policy, and expressed by administrators at Bayview High School.

In addition to the zero-tolerance approach to bullying, administrators at Bayview High School also mentioned the use of restorative justice when trying to resolve bullying incidents in the school. Both Brenda and Anthony, administrators at Bayview High School, described the role of discipline in enforcing the policy but also employing a restorative justice approach when possible. Anthony describes this approach:

we have a full-time restorative justice coordinator [...] but there have been instances where the bullying harassment has been so egregious that we have 
combined some kind of disciplinary consequences with a restorative process. So it depends on the circumstance and the level of offense

Restorative justice referred to a form of conflict resolution used the schools. The restorative justice approach involved teachers or administrations getting a restorative justice coordinator involved in the conflict between students. A teacher or administrator would notify the restorative justice coordinator, and then every student involved in the conflict, the perpetrator and the victim, would be required to meet and discuss the issue, with the ultimate goal of discussing how the student or students were harmed, and created solutions to the problem as well. The approach differs from the traditional zero-tolerance method in that students are not simply written up or punished for this actions. Participants at each school except Eastpointe High School mentioned the use of restorative justice approaches to resolving bullying in their school. It was apparent across each of these schools that utilizing this approach was very successful at resolving incidents and promoting social justice within their school. At Central High School, restorative justice was frequently mentioned by teachers. Ryan, who teaches at Central High, attributed the school's ability to resolve incidents to the restorative justice team. Ryan stated, "[...] they are a big part of fixing all of these problems in the building and they're a big part of getting the students to turn it around. I've never seen restorative justice until I came here and I think it's one of the best programs in the market." Ryan's comment illustrates the importance of a comprehensive approach to addressing bullying. Although restorative justice was still a reactive process, there were clear benefits to having a team devoted to addressing bullying or behavioral interventions in the school. Ryan recalls a specific instance with a student who was considering dropping out of school because of bullying: 
He did not believe that we could fix it. In his own mind he just didn't believe it. He said I already told one person, but he didn't tell anybody else. So, as his teacher, once I found out I went direct with the administration, the security team. I involved everyone that I could to make sure we could save the one kid and it worked. Sometimes it can't just be one person. And it takes a whole team to be able to fix these problems.

Ryan's example demonstrates that effective intervention can prevent more adverse outcomes, such as students deciding to leave the school. Caleb provides further support for a more team-based approach to intervention, referencing the shortcomings of other teachers when it comes to handling bullying issues: "so lots of F-words thrown around inappropriately in the hallway and there's a lot of shaking their fingers at them but not a lot gets done unless you talk to admins or the restorative justice coordinator about it." Earlier on in the interview, Caleb described his frustration with other teachers, who often would do nothing when witnessing bullying, and discussed the role of restorative justice in addressing bullying incidents. Even though restorative justice is the preferred method at Bayview High School, the approach to prevention is still framed using zero-tolerance language. In contrast, Mark, an administrator at Avenue High, focuses primarily on educating their staff through conversations and training. Mark describes the success of their approach: "use progressive discipline to have a conversation and alert them of the policies and expectations." Mark later went on to discuss their low rates of fighting and suspensions/ expulsions.

Understanding how incidents were handled within each school is an important piece of examining policy implementation practices among participants. Given that the anti-bullying policy conceptualized bullying as an issue that needed to be addressed using disciplinary action, the framing of bullying as a problem, and the interpretations of the 
school's policy influenced how teachers and administrators decided to address bullying incidents in their schools. In most cases, both teachers and administrators responded only when an incident occurred, generally through teacher intervention and notifying the administration. Multiple teachers and administrators across all five schools described the reactive process. For Zoe, a teacher at Avenue High, her approach involved reporting the incident directly or taking the student down to the front office and letting a school behavior specialist handle the situation. Beyond teacher intervention, which was the most commonly described form of intervention, students were also mentioned as examples in the classroom and the hallways of how to intervene when bullying does occur.

In terms of preventative approaches, Emily, who teachers at Bayview High, described her frustration with the lack of preventative strategies, and also with a lack of transparency from the administration in terms of addressing bullying:

we certainly don't talk in terms of preventative stuff and in response to that event that happened last year with the cyberbullying there was a group of people involved, students that were dealt with in way where nobody knew what was happening, staff didn't really know. The administration sort of did it kind of behind closed doors sort of thing.

To Emily, it was odd that teachers were unaware of how the cyberbullying incident was handled or resolved. Earlier on in the interview, Emily described another incident where a student was being targeted in her classroom, and she met with her students to try and resolve the issues. After addressing the problem in her classroom, she noticed that the bullying stopped happening in her classroom but noted that she did not know if the student was still being bullying and it just stopped occurring in front of her. Both Nathan and Patricia discuss how teachers may be left in the dark when it comes to addressing bullying. Similar to Emily, Nathan, who also teaches at Bayview, discusses how the he 
passed information about an incident to the administration, but did not always see what happened at the administrative level. To Nathan, however, this lack of transparency was attributed to confidentiality, and the he took comfort in knowing that the administration too incidents very seriously. In terms of administrative support for the LGBTQ community, Nathan stated, "our vice principal is a really big anti-bullying advocate especially for the LGBTQ community that they take pretty seriously and my experience with other schools hasn't always been the case." Patricia, who teaches at Dover High, described how in her opinion, it seemed as though students intentionally tried to prevent teachers from noticing bullying. Although she stated that immediately stepping in to intervene seemed to work when she did hear slurs regarding student's sexuality or gender, she also described what she called "subtle bullying" by saying, "I've seen the way students might talk to each other but they'll try and code their language so that does not appear to an authority figure to be bullying." In describing this tactic by students, Patricia illustrates one challenge teachers may face even in trying to take a reactive approach and shut down bullying when they hear students talking in the hallway.

Although most approaches were reactive, there were cases of proactive responses, and where an incident led to a prevention program as a response to the initial incident. When describing the outcome of an incident involving students making racist remarks, Anthony, the principal at Bayview High, stated, "we actually followed up with an entire approach with our freshman class on appropriate use of social media and bullying/harassment and racism, that stuff. So it can also branch out to people who weren't even involved in the incident but we use it as an education piece." Anthony's example demonstrates one of the ways that school administrators can take a negative 
event, such as an incident of racism in the school, and turn it into something positive, and use it as an opportunities to teach the entire cohort about how to treat their peers respectfully.

Thus far, in terms of the structure of the school, the responses of participants support the previous literature in illustrating the importance of messaging and relaying information about school policy. However, participant responses also reveal inconsistencies in teacher and administrator understanding of how the policy is enforced, the explicit language of the policy, and how to report or intervene when bullying does occur. The degree of variability within schools highlights the strength of individual factors such as personal beliefs and reading the student handbook, while also reiterating the role of structural factors such as delivering information regarding the policy at professional development meetings or trainings. In the remaining sections of this chapter, I assess participants' perspectives on bullying prevention, including accounts of the policy's effectiveness and reflections on what works and what does not work to prevent bullying in their school. I conclude this chapter with participants' suggestions for improving the policy.

What Works to Prevent Bullying? Policy Effectiveness and Strategies for Prevention

Understanding the perspective of teachers and administrators in terms of what works and what does not work to prevent bullying provides valuable insight on the impact that the anti-bullying policy has had on schools. Results from participant responses almost completely aligned with the administrative differences across schools, and teachers' earlier reports of training and awareness of the anti-bullying policy. Avenue 
High School and Bayview High School had supportive administrations, and a demonstrated commitment to bullying prevention through student-led anti-bullying campaigns. The expectation would be that at both of these schools, there would be an overall belief that the policy itself is not enough to prevent bullying. Across the other three schools, considering the differences in both administrator and teacher support for the anti-bullying policy, and LGBT youth, I would expect that there would be greater variation in perspectives on the anti-bullying policy, and especially since so few participants were trained on the policy and expressed awareness of the policy at Central High, Dover High, and Eastpointe High Schools. Results confirmed these differences with one exception, Avenue High School and Bayview High School differed in their approaches to bullying prevention. Although both Bayview High School and Avenue High School were similar in their commitment to LGBT youth and bullying prevention, they differed in their approach. The zero-tolerance approach at Bayview High School led some teachers to be more skeptical of the policy itself and suggest more proactive or holistic approaches to addressing bullying.

In order to address participants' perceptions of the anti-bullying policy's effectiveness, participants were asked questions regarding their perspectives on bullying prevention, policy effectiveness, and overall suggestions for improving the policy. In contrast to existing studies that point to the critical role that teachers and administrators have in influencing policy implementation results from participant interviews illustrate that in addition to teachers and administrative personnel, students, through studentcentered and student-led programs, have a substantial effect on bullying in the school (Coburn 2004, Coburn 2006). For policy effectiveness, each participant was asked the 
following question: in your opinion is the policy an effective policy to prevent bullying in your school? Responses suggest that the policy alone was insufficient, but a balance between student-led programs, adequate training for all staff, and clear expectations around behavior were essential to bullying prevention. Teachers from Avenue High and Bayview High described this notion below. At Avenue High, Zachary provided a very honest assessment of the difference between policy adoption and successful implementation. He stated:

I don't think policy in itself is ever effective. It sets the tone, and that's great and useful, but I've been in a lot of schools where it's been a policy but nobody follows through. We have a great policy, but it all comes down to the stem of having it being student centered, and students training faculty, and then students teaching other students on the culture and how is this unacceptable, otherwise it's just facts on a paper.

Zachary was one of a handful of teachers that pointed to the effect of student's on changing the school environment, ultimately contributing a great deal the school's ability to enforce its school's policy on bullying. Later on the in conversation, Zachary described how students were involved in making videos every year that talked about their personal experiences with bullying at school. When reflecting on the role of students, Zachary explicitly mentioned the role of student mentoring other students and faculty as well, Zachary stated, "all students should be able to teach their faculty and have that conversation." Zachary shares the perspective held by most teachers and the administrators at Avenue High, the importance of involving students in bullying prevention. Henry talked in more detailed about the effectiveness of their school's antibullying programs: "Regarding students contributing to the school's culture, more than one teacher at Avenue High mentioned the impact of their student-led anti-bullying 
programs: "I think having kids teach kids has been phenomenally significant in that and I think that again this like constant part of the dialogue and conversation among us is just saying we are a safe school." As evidenced in the literature on the role of GSAs, explicit efforts to support LGBT youth are most effective when supported by teachers and administrators in the school, fostering a sense of community and belonging among LGBT youth (Fetner et al. 2012; Grossman et al. 2009).

At Avenue High, allowing students to teach one another, share their experiences of bullying, and set the tone for bullying prevention through collaborations with staff and administrators were large contributors to their success in reducing bullying of LGBT youth. Previous research demonstrates the positive effect of schools supporting students' efforts to create a supportive environment for LGBT youth, with one study suggesting that schools that sponsor pro-gay events are more likely to have teachers who respond when hearing anti-gay words among students in school (Murphy 2015). Even though their reflections provide a lot of support for the importance of engaging students in bullying prevention, it is important to note that their responses also illustrate that the policy itself is not a primary contributor to the school's success with regard bullying prevention. Avenue High chose to create an anti-bullying campaign, which provides additional support for the role of not only teacher-wide trainings, but district-wide programs that are required by all schools, connected to the anti-bullying policy, and address bullying and the bullying of LGBT youth in particular.

In contrast, although Avenue High and Bayview High both had a well-supported QSA/GSA, in Bayview High, only the principal mentioned student-led programs. All three teachers and the vice principal focused primarily on the role of education, and 
creating a supportive classroom environment. In the responses of both administrators, however, they both agree that setting explicit guidelines for student behavior are critical elements of creating a supportive environment for their students. Brenda discussed how the policy sets the foundation for building an accepting environment by stating:

I don't know if I would say that this policy and the way it's written causes kids to say 'im going to refer back to the policy. I can't behave this way because of the policy $[\ldots]$ I think the policy is one where it supports and gives us a foundation but I think it's more than the policy that causes us to respect and appreciate each others differences. It's bigger than a policy.

Brenda's comment highlights two key points regarding policy effectiveness. First, she points out a piece about the policy itself that Nathan alluded to when talking about teachers in the classroom, the policy itself does not compel students to treat each other with respect. When discussing the policy further, Nathan described how the emphasis is on education and training for teachers, he stated, "yeah we can have a line in the handbook but $[\ldots]$ what's the process, who do you go to? If your friend is being bullied what do you do?" Nathan's comment solidifies Brenda's statement, "it's bigger than a policy", in demonstrating that protecting kids from bullying entails more than just words on paper, a sentiment expressed by several participants. Each response supports the existing literature pointing to the importance of education for students and staff regarding inclusive district policies (Kull et al. 2015; Poteat et al. 2015). The comment made by Brenda regarding the policy as setting the foundation is very key to illustrating the role of policy versus its implementation in each of the schools.

At Central High School, there was less of a consensus on the effectiveness of the school's policy. Caleb and Grace expressed that there was definitely more that could be done to improve bullying prevention in their school. Caleb was very explicit in stating, 
“the policy itself obviously isn't effective in my opinion because I see bullying happening all around but if you have more required trainings or programming in school I think that it is like a first step." As discussed in earlier points in the interview, Caleb was frustrated with the lack of teacher intervention and training, which comes through in his assessment of the policy's overall effectiveness. Grace also stated that more could be done. When discussing LGBT youth, Grace stated, "[...] our QSA students would like to see more to be done $[\ldots]$ is it enough? Probably not. Is it a move in the right direction? Definitely." Grace never gave specifics about the perspectives on the students in the QSA but her comment does reiterate that of Caleb's, there was more that the school could be doing to prevent bullying and the policy alone was insufficient. For Ryan, who was in his first year teaching at Central High, it was unclear whether or not the policy had been effective, but he did express a desire for increased visibility of the anti-bullying policy. Ryan stated, "I'm not really sure but I think that as a teacher I'd like to hear more about the anti-bullying policy. I'd like to have it posted. I don't see it [...] I think it should be posted for students to see on the doors and on the walls." Ryan's suggestion would not only enable all staff and students to see the policy, and become familiar with the language, but also would be a public display of the school's commitment to addressing bullying in the school.

As discussed by Kull et al. (2015), knowledge of the inclusivity of the school's anti-bullying policy has a positive effect on LGBT youth reporting of incidents. Thus, Ryan's makes an important observation regarding being aware of the policy as a teacher, and its visibility in the school. Similar to Central High, at Eastpointe High, both Molly and George revealed uncertainty in their assessments of the policy's impact on bullying 
prevention. For Molly, the school's administrator, it was difficult to say for sure if the policy had been effective because she could only reflect on the incidents that she had handled, which she stated had decreased over the years. For George, it was difficult to describe the policy as a success. When discussing a specific group of teachers, who provided a lot of push back against the school LGBTQ group in a meeting, George stated, "I don't know many other teachers in that part of the building that have explicitly presented themselves in meetings or in casual conversation to be advocates to LGBTQ students." George's reflection on that particular meeting reinforces the claims made earlier by participants, there is a considerable amount of difference among teachers in terms of their perspectives on LGBT students, factors which have an affect on if and how they support them. When reflecting on the safety of students and what works best to prevent bullying in the school, Georg expressed doubts about all students being protected in every location within the school. When discussing how he hoped that students felt comfortable coming to him or other teachers that create safe spaces, George described his concern in terms of teachers protecting LGBT youth:

[...] but it's just a really big building and there's a lot of variety in teacher attitudes and perspectives and just understanding, general understanding, of what it means to even be trans or to have a non heterosexual orientation.

For George, it was evident that the sheer size of the school, coupled with the variety of teacher attitudes and understanding of LGBT youth, was a barrier to ensuring that LGBT youth were protected. George's comment really reiterates the importance of effective training, not just on the school policy specifically, but also appropriate training on issues that students may face as sexual and/or gender minority students. George's concerns demonstrate that it really is not enough to just make sure that faculty are aware of the 
explicit language of the school's anti-bullying policy. His point is even more important given that he admitted earlier in the interview that he did not know what the anti-bullying policy said.

Participants highlighted creating an atmosphere or culture of respect through education, in addition to clearly laying out the expectations for behavior within the school as primary goals. When asked about best practices for preventing bullying, teachers' and administrators' responses at each of the five schools echoed a commitment to education, and suggested strategies for communicating expectations about bullying. When asked what his school should do to prevent bullying, or what strategies have/have not worked to prevent bullying in his school, Daniel replied:

I'd like to see teachers start out the year with conversations with their kids about class expectations, draw up a document together. This is our code of conduct, this is our bill of rights as students and as a teacher, here's how we're going to treat each other and address those issues that are usually hard to talk about. Like make sure, make a conscious effort.

Daniel describes a very community-based approach to addressing bullying. The notion of creating a contract between teachers and students, or having teachers read the policy aloud at the beginning of the year was echoed by other teachers. Daniel's comment illustrates the desire for increased accountability among teachers, and also how he would hope to see a more preventative approach taken by teachers as well. Earlier in the conversation, Daniel discussed the school's no tolerance policy. He stated, “[...] the instant no tolerance policy needs to be handled with some delicacy $[\ldots]$ we're teaching them how to be people out in the real world and they're going to naturally fall and part of our job to is to help redirect so its not punitive all the time." In contrast to Daniel's initial comments about the zero-tolerance policy, and his personal approach which included 
disciplinary action, Daniel recognizes that the punitive method that existed at his school is flawed and may not allow students to effectively learn from their mistakes. In effect, based on Patricia's earlier discussion of how students subtly try to code their language so adults do not perceive them as bullying others, the disciplinary method may encourage students to find more covert ways to bully.

In addition to Daniel's perspective on what does/does not work, Anthony, an administrator at Bayview, described that having students actively engaged and involved in any bullying campaigns had been the most effective. When describing this approach, Anthony admits that having an adult tell students not to bullying is not effective. He stated, "we have real life, real students, talking about the impact that it has had on them and students actually taking ownership and responsibility for that piece of the climate and culture here." Anthony continues on to discuss how, the student-led approach, adults become a support system that address issues when students bring them to the administration and send a message that the school is a safe place for them. Anthony's belief about what works aligns with his perception on the effectiveness of the school policy, he stated, "I'm not sure if the policy in and of itself is enough. I think you have to call it out specifically $[\ldots]$ having a lot of examples for staff and students about what is bullying and what isn't [...] I think it's helped us to be really specific about that." Brenda, who is also an administrator at Bayview, took the same position as Anthony, attributing their school's success to education, and student-led education. It appeared as though the perspectives of the administrators did trickle down to the teachers at Bayview, who did express similar sentiments regarding the importance of student-led programs. Similar to his comment earlier on the policy's effectiveness, Nathan discussed how 
students created a school-wide campaign to address bullying, and later commented on the policy's role in this process:

Is it going to deter a kid from doing it? No, I mean maybe if they know yes they will be suspended [...] I think there's other things to do besides a policy. I think there's a huge amount of education that needs to happen with students and with staff and students are educating students, and getting that out there.

Nathan highlights an important point about the use of the school's anti-bullying policy, he essentially states that the policy alone cannot be expected to change the school's bullying outcomes. Sarah, an administrator at Avenue High, emphasized that the lack of education was the driving force behind bullying in schools. Sarah stated, "I think its always really good to be proactive [...] cause a big part of why it even happens is lack of education, lack of knowing what it means." At Central High, teachers identified the restorative justice approach as the most effective approach to bullying prevention. For Caleb, a significant part of restorative justice was immediately interrupting bullying and then having the students brought into mediation. Caleb stated:

if I just address the entire problem and I use my voice they're going to have resentment toward me. I think they kind of need to figure out on their own through talking to each other what they're doing and what hurt it's causing.

From Caleb's viewpoint, restorative justice accomplishes two things. One, by requiring the students to go to mediation, they are forced to talk things out and come to a resolution. Two, by charging the students with the responsibility of resolving the matter, the student-teacher relationship is not strained. Grace agreed with Caleb, but expanded on his point to describe the outcome of only using discipline: "you exclude them for a few days and then they come back, nothing gets healed from that [...] it's hard to hate someone when you've sat across the table from them and seen them as a human being." 
Grace effectively describes the importance of holding students accountable for their actions, but doing so in a way that does not simply penalize them without given them the chance to reconcile their differences with another student. Similar to the point made earlier by Daniel, there were apparent advantages to being less rigid in terms of the zerotolerance policy and allowing students to learn from their mistakes and hopefully grow as a result.

Among these respondents, being proactive is paramount to bullying prevention, and the school's anti-bullying policy is not the primary focus when considering the best practice for bullying prevention. For Daniel, classrooms were spaces to create an agreement between everyone in the classroom regarding bullying and to set the tone early on that as a classroom everyone is going to be respected, and has a role to play in creating an accepting environment. This perspective highlights the importance of classroom culture and the role of teachers in creating classrooms free of homophobia, sexism, or any form of bullying and oppression, as discussed in the previous chapter. Sarah, a vice principal at Avenue High, brings up a commonly stated challenge among the teachers interviewed, the inability to be in each classroom and see all instances of bullying. In terms of what does not work, George, who teaches at Eastpointe High, questioned the role of " this is a safe place" stickers handed out at staff meetings, and expressed concerns about ensuring that LGBT students were protected in every space within their school. The variation between teachers in their approach to prevention and their perspectives on intervention are significant barriers to effectively implementing their school's policies. Findings from previous literature reiterate this notion, as LGBT 
students have reported that not all teachers respond to bullying incidents in school, as well as reporting that their teachers respond in different ways (Murphy 2015).

In addition to the classroom, Daniel also described the effect of representation on the bullying of LGBT youth. When asked to described any instances of bullying based on perceived sexual orientation, Daniel replied by describing the active role of the school's QSA and also discussed the role of representation: "and we have gender non-conforming administration and faculty members $[\ldots]$ there's faculty representation, administration representation, and student representation. I don't hear a lot of bullying around that." Daniel's response suggests that in addition to supportive classroom environments, having LGBT-identified faculty and administrative personnel may be important to creating a school environment that facilitates anti-bullying policy implementation. Although most participants spoke highly of restorative justice, and student-led programs, disciplinary action was mentioned by Patricia, who teaches at Dover High, and Molly, an administrator at Eastpointe High. When asked what strategies have worked to prevent bullying at their school, Molly responded, "I think suspending kids works. And putting out posters I think works. I'm not sure, hopefully it does. But in my mind, only what I see is what I know." For Molly, suspensions had proved successful. She continued on to discuss that suspensions serve to send a message to the other kids that what that student did was not okay, and it reinforces that message to the perpetrator, who later has to explain to other students why they were suspended.

In terms of policy improvements, participants suggested increased education about the policy, in addition to increased training on and visibility of the policy. Responses across all five schools demonstrate the differences in both teacher and administrator 
perspectives on how to improve the district anti-bullying policy. Teachers and administrators identified the importance of increased staff awareness of the school's antibullying policy, as well as clear expectations for student behavior. At Dover High, teachers emphasized the importance of being explicit when talking about bullying, discussing the policy with students more than once, and having well-defined guidelines for reporting. When asked to provide suggestions for improving the policy, Anthony Patricia articulated the value of moving past an approach that focuses too heavily on consequences for behavior. Anthony stated:

it falls into a kind of a big giant category of consequences for behavior [...] I think a more proactive approach is better [...] making sure that we're doing a lot of stuff up through elementary and middle school around this topic and not just relying on a consequential kind of approach to student behavior.

In his response, Anthony conveys benefit of not only being proactive within his school, but also replicating the same approach and educating students before they enter high school. At Central High, Caleb and Michael provide examples of proactive approaches that schools can take toward preventing bullying. For Caleb, communication through mentoring and creating allies for LGBT youth was a place to start, in addition to creating a leadership team exclusively devoted to bullying. Michael, on the other hand, suggested training students as a cohort, beginning with freshman, before school year starts:

there should be like two weeks where you just have the freshman, they don't really go to class, you just treat them like a cohort. And you just let them have it right then. Lots of intense expectations including things like bullying and academic expectations but also bullying, how to behave, this is how we behave in this school $[\ldots]$ positive discipline $[\ldots]$.

Michael continued on to describe the current challenge with creating effective strategies for prevention, the variation in classroom expectations. When talking about the the need 
to create role models through student training, Michael, stated, "right now it goes from teacher to teacher, these kids bounce around, and something that's allowed in one room is not allowed in an other room." Michael's comments reiterate the value of training teachers and students on bullying expectations, and the overall language of the school's anti-bullying policy. Regarding the language of the policy, not every teacher believed the policy was written in a way that accommodated teachers. When asked to offer suggestions for improving the policy, Patricia expressed her frustration with district policy in general: "what's the problem and what's the consequence? [...] Often what you find in district policy is this very long winded legal looking writing and that ends up getting ignored because nobody understands what that means in actuality." Patricia highlights an important aspect of understanding school policy. Do teachers understand the language of their school's anti-bullying policy?

Participants' response suggests that some teachers do but there are many who do not. Patricia was the only participant to bring up the actual wording of the policy, illuminating a potential pitfall of the way that district policies are written, but also offering a potential solution to the problem of teachers not being able to comprehend the language of their school's policy. Eric, who teaches at Eastpointe High, provides support for Patricia's claim. When suggesting that teachers become well-versed on the policy, Eric reflected on his own ability to recite the policy, stating, "I know I've read it [...] our handbook a million times but I couldn't quote a single piece of it other than a general idea of anti-bullying or harassment is you can't harass or bully anyone based on race or gender or anything else." Similar to Eric, it is likely that many teachers have read their school's handbook but that does not translate into a working knowledge of the policy, 
ultimately hindering their ability to effectively intervene for students and participate in implementing the policy.

Emily, who teaches at Bayview, suggested that the policy should be more available, that teachers should be more knowledgeable, and that students and teachers should be required to write out the policy together at the beginning of the year. Her response is very similar to that of her colleague Daniel, and highlights the issue of accountability, which was an important topic for participants. Zoe, who teaches at Avenue High, offered a contrasting viewpoint. In her opinion, teachers, and especially newer teachers, are overloaded with responsibilities and are not properly integrated into the school environment. She stated:

I haven't been trained on it, so it's a matter of reading through the manual if it's even in there which is one of many many things that teachers have to address at the beginning of the school year. I think it's something that is kind of endemic here $[. .$.$] There's a lot of figuring things out as a new teacher and finding it$ yourself.

Zoe illuminates a challenge that is distinct to new teachers, balancing her new responsibilities and also trying to become immersed within the culture of the school. Zoe elaborated on this process by describing the limitations of training at the beginning of the year. She stated, "there isn't really time at the beginning of the year for training. We only have one and a half days a year where we are required to meet. There's a list of things that need to be covered, some are some are not [...] It's a time thing." Zoe's beliefs are very similar to Caleb's, who works at Central High. Caleb previously expressed his frustrations with optional trainings, and revealed that although he was aware that he lacked knowledge of the policy, he did not have extra time in his schedule to attend a training during his professional leave time. 
In addition to time constraints limiting teachers' ability to receive training, George questioned the impact of training all together, reflecting on mandatory trainings on diversity. While reflecting on the topic, George grappled with the conflict between the personal benefit he gained from the diversity training, and the lack of growth he saw in his colleagues who also attended the training. He stated, "I hear colleagues of mine say things that are really derogatory about what we do and how no one can tell them they have white privilege and it doesn't exist." George went on to express his concern with adopting a similar training around LGBTQ issues and allyship. He wondered if the training would have the same outcome and teachers would ignore the content.

In terms of training specific to LGBT youth, when asked if there had been any trainings or programs in the past year on bullying prevention based on perceived sexual orientation, Rose, who teachers at Avenue High, suggested increased accountability on the administration regarding teacher training. She mentioned that she would love to attend the equity training, but has not been held accountable in terms of going. She expressed that accountability has to come from leadership, and stated, "Like a Vice Principal saying hey you need to go complete this and do this. That hasn't happened. It could be as simple as that." Rose also suggested tracking who attends trainings and taking the time to get in touch with the teachers and staff to make sure they receive training. Rose described an important challenge that faced as they tried to increase their knowledgeable of their school's policies or protocols. While it was apparent that Rose preferred to attend the district-wide equity training, she had never been held accountable for attending a meeting. Who should be held responsible for ensuring teachers attend trainings, and what kind of message does it send to teachers if they are not encouraged to 
attend school trainings? In addition to administrators' having an effect on how teachers understand bullying as problem, it may also be the case that if principals and vice principals do not hold teachers accountable for attending a training, they send a message to the teachers and staff that the training must not be that important.

Participant responses demonstrate that the school structure, as it pertains to antibullying policy, is wrought with barriers to effective policy implementation. Although administrators due play an intricate role in problem framing, review of teachers' and administrators' understanding of bullying as problem in their school reveal inconsistencies, both within and across schools. Some teachers were very aware of the bullying taking place in their school, as well as the issues affecting LGBT youth, such as Caleb at Central High, while others working in the same building were unware if the school has transgender students, let alone any instances of bullying that have occurred among LGBT youth in the school.

As a whole, the findings on school structure reveal the plethora of challenges that teachers and administrators face when trying to protect their students. Even though the policy was available through the district website, the state website, and there was language in the student handbook on bullying protocol, participants demonstrated differences in their knowledge, awareness and receipt of training on the anti-bullying policy. Administrators seemed to be the ones receiving training on the anti-bullying policy at the district level, while teachers are poised with the task of finding ways to be aware of trainings available in their school, and then making the time to attend the trainings. It was apparent based on participants' suggestions that that the policy was not visible enough, and the language not only contained too much legal jargon, but also as 
too focused on consequences and as ultimately reactive in nature. Avenue High School and Bayview High School, despite their similarities, illustrated the importance of administrator framing of bullying a problem. Administrators at Avenue High School portrayed bullying as a general issue but not a concern in their school, which had an overall impact on how teachers in the school viewed bullying. Despite student-led and school-based trainings on anti-bullying, there were clear disparities in teacher training on bullying, and the anti-bullying policy, drawing attention to the issue of mandatory versus optional trainings, as well as the availability of information on school trainings. In contrast to Avenue High School's approach to the policy, teachers at Bayview High School were aware of the zero-tolerance approach to bullying, and it was evident that the administration's perception of bullying as a disciplinary issue translated to teacher's understandings and approaches to bullying in the school. However, teachers at Bayview ultimately expressed some discontent with the discipline based approach, offering solutions through suggesting more holistic or proactive approaches to bullying. Accounts from teachers and administrators at Central High, Dover High, and Eastpointe High School revealed greater variability in understandings of bullying as an issue but an overwhelming lack of training and knowledge of the anti-bullying policy.

In terms of additional approaches to bullying, restorative justice was a positive example of schools using team-based strategies to get students to be more actively involved in resolving conflicts when they did arise in the school, but there were limits to this approach given it was still a reactive strategy. As far as LGBT youth were concerned, very few participants received training on preventing bullying based on perceived sexual orientation, revealing a significant gap in teachers' ability to help all of their students. 
The following chapter explores these challenges associated with trying to protect LGBT youth by demonstrating that factors such as limited social networks for teachers, the prioritizing of race over other identities at the district level, and the prevalence of cyberbullying created barriers to protecting LGBT youth. 
V. PROTECTING LGBT YOUTH FROM BULLYING: FACTORS THAT SUPPORT AND PREVENT TEACHERS FROM ADVOCATING FOR LGBT YOUTH

The previous chapter examined how the policy, the language of the policy, and its interpretation influenced teachers' and administrators' experiences with training on the anti-bullying policy, training specific to LGBT youth, and overall perceptions of bullying as a problem in their schools. Assessing the current school structure as it pertains to policy implementation revealed several gaps in terms of enabling teachers to provide support to LGBT youth, intervene when instances do arise, and attempt to prevent bullying based on sexual orientation and gender identity. In this chapter, I argue that in addition to the effects of the policy on the school (i.e., training, perceptions on bullying, and approaches to bullying), limited support through social networks, competing district priorities, and issues related to cyberbullying prevented teachers from advocating for LGBT youth and preventing the bullying of LGBT youth in their schools. Similar to the participant accounts of the policy, participant interviews revealed variability in terms of teachers' strategies and the degree to which classrooms and the school as a whole served as supportive environments for LGBT youth. All of these factors affected teachers' ability to intervene, and their perceptions of their ability to help students who did experience bullying. Despite these challenges, I also demonstrate that teachers did manage to find ways to protect LGBT youth, through community-building in their classrooms, and leveraging the support of other teachers when possible. The first half of this chapter describes the factors that allowed teachers to advocate for LGBT youth in their classrooms and throughout the school. In this section, the role of teacher intervention, administrative support, and community-building strategies are discussed. 
The remainder of the chapter outlines the challenges that teachers faced when trying to protect LGBT youth from bullying, and describes the role of limited social networks, the districts' focus on race over other identities, and issues related to cyberbullying.

Supportive Factors: Teacher Advocacy, Community Building, and Administrative Support for LGBT Youth

As outlined in the district policy, teachers were responsible for reporting any incidents of bullying that they witnesssed, and any instances of bullying that they were aware of. It was evident through participant responses that teachers were aware of their individual responsibility to students. For some, this responsibiility was more than simply providing reports of bullying. Daniel, who teaches at Bayview High School, stated, "it's got to be teacher by teacher $[\ldots]$ yeah the administration can put something into place and they have but they're not in the classroom everyday with every single kid so it has to come down to us at some point." The comment made by Daniel is important to consider because of his focus on teachers but also the distinction he makes between teachers' and administrators' role in enforcing the school's anti-bullying policy. Nathan, who also teaches at Bayview shared a similar sentiment to Daniel. Nathan stated, "I wouldn't say that the district saying we have a zero tolerance policy affects how I teach in my classroom. I would say my morals about being an educator.” For Nathan, neither the availability of the policy, nor it being a zero-tolerance policy, motivated him to interrupt bullying or talk to students about bullying. Nathan's response illuminates the role of teachers and their beliefs as significant contributors to the enforcement of the school's policy, at least at Bayview. As expressed in previous studies, teacher beliefs influence not only their interpretation of a problem but also how they choose to implement school 
reforms (Coburn 2004; Marz and Kelchtermans 2013). Specifically, he suggests that since teachers are working closely with students everyday they are in a sense responsible for what happens in their classroom and that administrators do not have the same type of responsibility.

In contrast to Daniel's beliefs, Nathan, who also teaches at Bayview, commented on how teachers' reach in prevention only goes as far as channeling incidents up to the administration. When asked how the policy was enforced in his school, Nathan responded by stating, "generally, they do a bunch of research. They look up stuff, have text messages and Instagram accounts. They interview witnesses and bystanders, and bring the kids in $[\ldots]$ it generally happens at the administrative level." Later on in the interview, Nathan admitted that it was challenging to pass things on to administration. Since he took a parent-type role with his students, and wanted to help/protect then. Overall, he did feel handing incidents off to the administration was the best option for his students. Nathan stated, "and with a class of 30 students we don't have that confidentiality ability $[\ldots]$ you want to protect that kid and counseling and administration have the ability to do that. There's only one of us and 35 kids." When we consider the two comments together, it is apparent that although teachers were able to have a considerable impact on the school environment through their advocacy in the classroom, they were limited in their ability to ultimately resolve bullying incidents when they did occur. It was evident that the administration shouldered the responsibility in terms of notifying parents, or getting the counseling team involved to address the issue. Despite these limitations, multiple teachers across the district discussed the importance of teachers' willingness to have difficult conversations with their students, and create a 
culture of acceptance early on in the classroom through community building. Several participants were engaged in community building practices with their students. Teachers at at Bayview High, Central High, and Eastpoine High School shared their experiences in the next section.

Community-building in the classroom

The use of commnity-building techniques in the classroom is one aspect of the teacher-student relationship that helps to establish trust, set explicit rules for the classroom, and actively engage students in practices that support positive relationships bewteen students and discourage bullying (Doll et al. 2011; Frey et al. 2011). Daniel and Caleb discussed their strategies for making the classroom a supportive, and inclusive place. Daniel stated, "I ask preferred pronouns of all my students on the first day of school. Preferred first name and preferred pronoun so we can get that sorted out and I cannot mess up a whole ton of times. So they're getting called and addressed the way they want to be." Daniel's approach allows students who do identify as LGBT, or gender non-conforming, to feel as though the classroom is a place where they will be respected, and recognized for who they are, regardless of their identity. In terms of LGBT youth, Caleb, who teaches at Central, also took the time to ask his students about their experiences as sexual and/or gender minority students. He stated, "I've have a lot of students that have been discriminated for their sexuality or gender identity so I wanted to know what's being done and I hear their stories about their classrooms and their interactions with their teachers." Caleb's response relates back to the importance of teachers serving as advocates. After listening to his student's experiences of discrimination, Caleb decided to ask why teachers were not properly trained on issues 
related to LGBT youth. He could have taken the approach of simply reporting the instances described by students, but instead, hearing that his students were experiencing discrimination warranted addressing issues of teacher competence and training.

In efforts to create a classroom community that supported all students and discouraged bullying, teachers created blanket rules or guidelines for their classrooms. For George and Caleb, this meant making sure that students knew the use of oppressive language would not be tolerated. Caleb's approach is described below:

My only rule is 'no racist, sexist, homophobic or otherwise prejudiced remarks are tolerated'. If somebody says it they have to write an apology letter and explain to the rest of the class why their comment was racist, sexist, or homophobic by doing research on the issue and then we try to create a safe environment in the class.

For Caleb, the first few weeks of the term were devoted to community-building, and creating a classroom environment where students would not engage in behaviors or use language that could harm their fellow students. Emily took a similar approach, and tried to develop strong relationships with her students, in addition to encouraging her students to have strong relationships with one another. When reflecting on this process, Emily stated, "anything from a lot of get to know you activities in the beginning of the year and then lots of social learning where they sort of are put together in a multitude of ways to break up the cliques and finding common ground with people." George, at Eastpointe High, emphasized that the classroom was a professional environment and encouraged students not to use homophobic or racist language in their own professional environment or any environment. Each participant's response relates back to the restorative justice approach taken by several schools within the district. By sending the message to students that the classroom is their community, it reinforces the notion that they should not try to 
harm, oppress, or bullying other people in their community, and that they all have shared interests as a group. The fact that George and Caleb both worked in schools that were not as supportive of LGBT youth demonstarted the value of individual teachers choosing to step in and advocate for LGBT youth, in the absence of not only structural support through training but also administrative support. In the following section, I demonstrate that administrators play an integral role in establishing school environments that support and encourage LGBT youth.

\section{Administrative support for LGBT youth}

While teachers are able to create communities in their classroom, the ease with which they can accomplish this is in part due to the efforts of the school administrators. Results from the previous chapter demonstrated that administrators were powerful actors in framing bullying as an issue in the school, but also identifying LGBT youth as an important subset of the student population. Most effectively illustrated at Avenue High and Bayview High School, the promotion of QSAs and willingness of the administration to incorporate LGBT youth into anti-bullying campaigns articulated to teachers at both schools that LGBT youth were an important part of their school's community.

Throughout participant interviews, it became apparent that in addition to teachers' tactics in the classroom, administrative support for students, and LGBT youth in particular, was an important factor in determining teachers' ability to intervene when students were bullied. Research on bullying prevention, and LGBT youth specifically, highlights the importance of administrative support in creating a safe environment for students. Fetner et al. (2012) and Griffin et. al (2004) illustrated that administrators often serve as the gatekeepers for the acceptance of a LGBT youth support groups such as GSAs. School 
administrators are responsible for not only policy enforcement, but also relaying messaging about enforcement procedures, protocols for reporting incidents, and setting the tone for the culture of the school (Coburn 2001). In the present study, the support of principals and vice principals was critical to ensuring the visibility of the LGBT youth population, and building the social capital of teachers and administrators in terms of intervening in bullying. Participant interviews indicated that administrators helped in the following ways: 1) ensuring that LGBT youth were represented in anti-bullying programs, 2) allowing LGBT youth to have a voice in the school, 3) making teachers and staff felt supported in their efforts to protect LGBT youth and all youth effectively, and 4) fostering community and creating an overall school culture of equity and acceptance.

Regarding LGBT youth representation, Avenue High and Bayview High School served as model schools within the district because of their commitment to their LGBT student population. Both schools had a well-supported and thriving QSA/GSA, actively involved students in its anti-bullying programs, and had administrative personnel who were active in promoting equity and inclusion for the LGBT student population. Teachers at Bayview High stated that there was a relatively large LGBT youth population at their school. At Avenue High, although teachers and administrators did not mention the size of the LGBT population, participants frequently mentioned the strength and size of the school's QSA. In interviews, teachers at both schools mentioned the supportive role of the administration. Zachary, who teaches at Avenue High, described how the administration's support of the students was the driving force behind their ability to empower students. Zachary stated, "directly from our leadership at the top [...] students can come in at any time $[\ldots]$ hiring faculty who honor students' voices, from the 
administration to the teachers, empowering our students [...]." Zachary focuses on the open-door policy of the administration, which allowed students to come into an administrator's office at any time and express their concerns or share their ideas. Student empowerment was a staple of their administrative platform. Daniel also discussed the positive influence of the support provided by the administration, focusing on the impact administrative support had on the QSA. Daniel stated, "we have new administration, pretty much from top to bottom [..] and that has shifted a focus that has made a difference [...] And they've given a lot of voice to the QSA organization, a lot of power to them, and it's been successful." Daniel followed up his statement by adding that the new perspective of the administration was part of the reason that derogatory remarks were not hear in the hallways, to the same extent that remarks were made at other schools, in his opinion.

The comments made by Daniel and Zachary both described the strength of their administration. For both teachers, the example and leadership from coming from the top down to the teachers, staff, and students had a positive impact on their perspectives on the schools' values and support of LGBT youth in particular. Zachary also discussed how the teachers' and administrators' desire to see students advocate for themselves, and make their cases to the administration or even the district, led to their QSA being intricately involved in training students to respect each other. Zachary stated,

a huge group and a powerful queer straight alliance, who go around to classes and are giving a $[\ldots]$ presentation about how they identify and some of their issues and really want a trans gender bathroom [...] the principal was really supportive and progressive and said if you want this to really happen you need the buy in of your students and faculty. 
Henry, who also teaches at Avenue, described how transgender students received a lot of support from their peers. Henry described one instance at a school assembly, and stated, "this other student would speak at assemblies in full makeup and at my high school you would beaten to a pulp before we got out of the parking lot and he got a standing ovation." Henry's candid remarks provide an overwhelmingly positive example of how transgender students were supported at Avenue, contrasting their experience in high school with those of their students. The comments made by both Henry and Zachary provide further support for the level of commitment to the LGBT student population by the administration at Avenue High School. The combination of the supportive administration, evident by Mark's desire to help support students with gender neutral bathrooms, and Jane's dedication to incorporating LGBT youth in the school's antibullying campaigns, were aspects of Avenue High School's administrative climate that facilitated a lot of the success at the school in terms of reducing bullying and protecting LGBT youth in particular.

Similarly, at Bayview, Emily discussed how the administration took the time to make their commitment and respect for the transgender student body very evident. Emily talked about different types of announcements that were made regarding LGBT youth and stated, "[...] making those bathrooms more visible and making sure all the students know about it $[\ldots]$ calls to never divide your class by genders and maybe at the beginning of the year everyone should just say their preferred pronoun.” The direct focus on transgender students may in part be due to the visibility of LGBT students in terms of numbers, but nonetheless demonstrates that transgender students were given adequate support from the school's administration. 
In addition to teachers referencing the support of their principals and vice principals, teachers and administrators also noted that the district played an important role in school culture. In addition to the role of the district in problem framing and sensemaking for teachers, principals, and vice principals, participant data also revealed that the district contributed to the social capital of administrators (Coburn 2004; Benford and Snow 2000; Coleman 1998; Marz and Kelchterman 2013). For Mark, an administrator at Avenue High, support from the school district was essential to his ability to create a positive school environment for LGBT youth. When asked about how the anti-bullying policy affected the way he interact with students he responded by talking about how years prior he would not have felt as confident pursuing initiatives to improve LGBT youth inclusion, such as gender neutral bathrooms, in the school but now felt that he had the support needed to do so. Mark stated:

And I think ten years ago I would have been really scared of that and now I feel supported to help them [...] I trust the district will support something like this. And the community's awareness has raised enough. So I would have always wanted to do it I just would have been more nervous about it. Mark's comment illustrates an instance where an administrator's beliefs regarding supporting students, and providing a safe space through an inclusive bathroom, are not enough to ensure that students actually receive the support they need. Regardless of Mark's beliefs, the district making it okay to support LGBT students was the main reason why Mark felt he could go ahead and help them. His example demonstrates that one aspect of implementing school anti-bullying policies involves an attitude shift from the top-down. Grace and Molly, a teacher and administrator at Central and Eastpointe High High, provide additional support for this claim. Grace stated, "instead of progressive educators having to advocate for students, it's the district saying "hey we 
really want to honor everyone who is going to our schools." Grace expressed her excitement at witnessing the change in the public school system over the years. Her comment emphasizes that although teachers can lead the charge, as suggested by Daniel, and Caleb, it is even more powerful to have the school district publicly support its students, opening the door for progressive teachers to do even more, without the fear of scrutiny or pushback.

Building on the findings of Marz and Kelchtermans (2013), not only teachers' but administrators' beliefs regarding the effects of bullying were important aspects of their decisions to support and enforce school policy. When asked if the policy can or should be used to protect against bullying based on perceived sexual orientation, Molly, an administrator at Eastpointe High, described her beliefs on bullying and the policy: "As long as you tell me, if it's brought to my attention we will enforce the policy to the highest degree [...] I hate bullying [... The policy matches up with how I think so it's a good thing. In my mind. It helps me." For Molly, being able to enforce the policy and provide the appropriate level of discipline when bullying does occur was encouraging and reassured them regarding their personal beliefs pertaining to bullying. In addition, Molly continued on to discuss how without the support of the district superintendent, the school would not be the same. Molly stated, "for me, because our superintendent endorses and supports the policy, I think it holds a lot of weight [...] I'm glad they support it and tell us leaders it's important and generate and provide the information like the powerpoint, the video and stuff and the policy to make sure that it's visible [...]." Molly went on to say that without the district's support of the anti-bullying policy, the school's outcomes could be solely dependent on who the administration is at the school. 
Molly's comment is very indicative of previous literature demonstrating how the district can positively impact the culture of a school. When reflecting on how their school might be without the support of the superintendent, Molly recognizes that their school may not be as effective in implementing the school's anti-bullying policy. Though this could be the case, Molly's statement reflects a perception that the responsibility of effectively implementing a school's policy falls on individual administrators. In perceiving policy implementation as simply individually based, Molly misses the role of the structural environment created by the district policy, and the important role that training, knowledge, and exposure to the anti-bullying policy has on the abilities of both administrators and teachers to be involved and engaged in the protection of their students by enforcing the policy. Considering the role of administrative support in protecting LGBT youth revealed that both the district and the school administrators made efforts to send positive messages throughout the district about the importance of LGBT youth. Teachers and administrators were very cognizant of the support that their administrators provided in terms of supporting gender-neutral bathrooms and giving QSA/GSAs a lot of support and opportunities to express their concerns throughout the school. The fact that teachers from Dover High School and Eastpointe High School were not among participants expressing gratitude toward the administration likely reflects differences in the support of the administration at those schools, and also reflects the comments made in the previous chapter regarding potential distrust in the administration and an overall lack of training on the anti-bullying policy. The following section examines the factors that prohibited teachers from supporting LGBT youth in their schools. 
Challenges to Protecting LGBT Youth: Limited Social Networks, Focusing on Race, and Cyberbullying

In the previous chapter, participant accounts of their experiences with the policy revealed several barriers to policy implementation. The fact that many participants were not familiar with the anti-bullying policy, and reported limited to no training on the policy or preventing LGBT youth from bullying is an important part of understanding the challenges that teachers faced with trying to protect LGBT youth in their classrooms and schools. Previous literature suggests that district policy frames problems in schools, and structures how teachers and administrators learn about the policy, protocols for implementing the policy, and network with one another to leverage resources for implementing the policy (Coburn, Matia, and Choi 2013). In the present study, the absence of consistent knowledge, exposure, and training on the anti-bullying policy resulted in a school environment where teachers were not given opportunities to network with one another and learn about the policy from their colleagues and administrators. The lack of opportunities for training and discussing the policy is problematic, and contributes to barriers when it comes to protecting LGBT youth. As demonstrated by Greytak, Kosciw, and Boesen (2013), the use of professional development training workshops can have positive effects on teachers' perceptions of intervening in homophobic remarks, being empathetic towards LGBT youth, and increasing teacher confidence in their ability to intervene. Since the teachers in the current study were not all trained on the policy or LGBT youth, their challenges to protecting LGBT youth would be anticipated.

In this study, one evident challenge to implementing the school anti-bullying policy and protecting LGBT youth existed in the language of the policy itself. The policy 
includes LGBT youth in a long list of protected classes, without ever discussing how to address the unique needs of each protected class. Regarding training, districts were not required to create new training programs. Instead, districts were encouraged to incorporate bullying into existing programs, the district policy never discusses this aspect of the state law. Without explicit instructions on how to prevent or address bullying, schools were left to decide for themselves which protected classes were important to focus on in their bullying prevention efforts, how to train teachers, and what protocols to put in place regarding bullying. Analyses of the policy structure revealed that teachers have limited working knowledge of the policy and the issues that pertain to LGBT youth regarding bullying. These limitations were further compounded by competing district priorities when it came to prioritizing protected classes. In this section, I argue that the district's privileging of race over gender and sexuality, in combination with limited working knowledge of intervening in bullying, and cyberbullying, created significant challenges for teachers seeking to protect LGBT youth. In an environment where the district policy and administrative framing could have created social networks for teachers to gain skills and discuss the policy, teachers' experiences revealed very limited networks of support for learning about the policy and LGBT youth.

\section{Using networks to build social capital}

Due to a lack of formal training on the anti-bullying policy, teachers were not given formal opportunities to network with each other or administrators in order to learn about or discuss the anti-bullying policy. However, some individual teachers still chose to utilize their social network. Individual's choice of utilizing a social network resulted in 
some teachers gaining information and tips from each other in order to attempt to best protect LGBT students. For some teachers, this was evident in their discussions with other teachers in the lunchroom or teachers' lounge, where they talked about how to address bullying in their classrooms. For others, teachers who had identified themselves as members of the LGBT community served as resources for understanding the issues that LGBT youth faced in school. As a whole, each of their examples illustrate how some teachers found ways to build social capital for protecting LGBT youth. When applied to anti-bullying policy implementation, social capital includes both the social networks teachers create or have access to, any information that is relayed to them regarding bullying as a problem in their school, the anti-bullying policy itself, and any specific procedures pertaining to intervention (Coburn and Russell 2008; Coleman 1998; Smylie and Evans 2006). Coburn, Matia, and Choi (2013) illustrate that district policy structures teachers' networks through determining the information that they receive about a policy, and creating opportunities for teachers to meet with other teachers and discuss the policy at meetings. Participant reflections on their discussions of bullying with others revealed the role that social capital has in allowing teachers and administrators to feel capable of intervening, in addition to bolstering confidence in their interventions (Marz and Keltchermans 2013). Results of asking teachers and administrators if they discuss the policy or and/or bullying with others yielded a variety of answers. There were some teachers who were able to garner information on intervention and LGBT youth through their peer networks. For these teachers, competence was built through conversations with other teachers deemed to be experts on the topic. For others, discussions with other teachers and peer-networks provided valuable insight for reporting and interventions. For 
George, a teacher in Eastpointe High, social networks were integral to his understanding of how to handle bullying in the school. When describing conversations in the teacher's lounge he stated "students maybe have recently decided they identify with a different gender [...] just sort of problem solving about what their role should be and how other teachers would react or support students." George's response really hones in on the importance of teacher networks, especially for protecting LGBT youth, as some teachers may be unaware of how to address gender pronoun or name changes in the classroom.

Later on in their discussion, George also talked about how LGBTQ-identified faculty served as useful resources, which allowed other teachers to have open conversations about issues that relate to LGBT youth and get advice. In George's response it is apparent that his peers created a space where they could share information and problem solve together. The one LGBTQ-identified faculty members who George mentions served as a primary resource on LGBTQ youth issues. In contrast to Coburn and Russell (2008), who found that teachers identify policy experts in their school based on professional development meetings, in the absence of structural support through trainings, teachers made sense of the policy through conversations with others, and building social capital through their peer networks and then make decisions regarding bullying based on the consensus of their peers. Daniel, who teaches at Bayview, also reflected on their peer networks and the utility of lunchroom conversations: "I draw a lot on the experience of my peers and they are the ones that I associate with the most $[\ldots]$ we do discuss ways to interject ourselves in situations, how to shelter kids off who feel as though they are getting attacked." While Daniel and George did have supportive peer 
groups, George was very cognizant of the privileged position he was in, both in having like-minded colleagues and a supportive teacher network.

Although there were some teachers would found supportive networks, other teachers at Bayview High, Dover High, Central High, and Avenue High expressed limited conversations with their peers, referencing staff development meetings or never conversing with other teachers. Across participants there was a considerable degree of variation in terms of frequency of communication with others, Patricia and Eric could not recall any conversations about bullying or the policy, while Michael and Grace could specifically recall conversations with peers through their department or at staff meetings. When describing the dynamic in her department, Grace stated, "I think as a department we aren't specifically talking to each other like hey there's this specific policy, but we talk about things regularly about how to include LGBTQ voices and what we can do within the department and we work together as a department." Grace's comment illustrates the strength of her network of teachers, all teaching similar courses, who actively find ways to ensure that LGBT students are represented in the classroom. The level of dissimilarity in communication among teachers is surprising and draws attention to the importance of all staff and teachers being aware of professional development meetings, in addition to being encouraged to talk to one another about school policies.

Each participants' reflections on communication with others are important towards understanding how social capital is generated within a school, as previous literature suggests that teachers engage in collective sense-making through professional development seminars and discussions with other teachers. Following Marz and Kelchtermans (2013) and Coburn (2001), teachers make decisions around whether or not 
to support a policy and how to implement school policies based on their discussions with others. Therefore, the differences evident across all five schools suggest that teachers may not be engaging in collective sense-making and instead deciding on their own if and how they will support their school's anti-bullying policy. For example, recalling Patricia at Dover High who reported little conversation regarding bullying in general or reporting, and George at Eastpointe High, who reported discussions with their colleagues, George had more social capital, and the social support provided through conversations with other teachers seemed helpful in developing strategies to prevent bullying and receiving guidance on what to do when an incident occurred.

In addition to discussions of bullying, teachers' social capital is also dependent upon the availability of resources to effectively intervene, including support from administrators. When discussing how the policy was enforced in their school, George, who teaches at Eastpointe High, expressed his challenges with advocating for students. In particular, he mentioned that although he frequently relied on other teachers for support, he was less inclined to talk to the administration when issues arise pertaining to LGBT youth. After divulging that he did not completely trust his administration, George discussed how if there was a safety issue with a student, he would go directly to the administration. However, when it came to talking to students, George stated, "I would probably go the route of reaching out to other teachers than administration [...] I just have never been given the impression by them that they are particularly comfortable discussing issues of LGBT youth." George was very candid in expressing his frustrations and concerns with the school's administration. Previously in the interview, George described an event where a LGBT-identified student was not receiving enough support 
from their teachers. George stepped in, was able to talk to the student's parent, and resolved the situation. Reflecting upon this incident, George mentioned that we only spoke to one parent because the student was not out to both parents but was unsure if the administration would have been as discrete with the matter. He stated, "I would be concerned that our administration would be comfortable outing students because they would feel the parents would have the right to know [...] I don't personally believe that." George's example reinforces the role of teacher's beliefs in their decisions to adhere to school policies but also is a prime example of how teachers within the district found ways to advocate for their students despite limited resources. In addition to the limitations created by the lack of social networks for understanding the school's policy, teachers also expressed challenges due to the district's decision to prioritize race over other identities within the school district. In the following section, I demonstrate how the district's messaging on the important of race further minimized the time and resources available to teachers for protecting LGBT youth from bullying.

\section{Focusing on race}

LGBT youth were one of several protected classes listed in the state law and district anti-bullying policy. Although enumerating sexual orientation and/or gender identity/expression is a vital component to protecting LGBT youth, the enumeration alone is not sufficient to protect LGBT youth from bullying (Kull et al. 2015). Just as the district policy framed bullying as a disciplinary issue, the district also framed race as a primary issue in the school district. The decision to focus on race in the district was not without merit, Central High School, Dover High School, and Eastpointe High School 
were very racially and ethnically diverse schools. Central High School was a minority white school, while Bayview High and Avenue High School were majority white schools. Both Eastpointe High and Dover High School had fairly even distributions of white and non-white students. Contextually, the state and district had a history of conflicts and discrimination between whites and people of color. Thus, the decision to pursue race as an issue in the schools was an appropriate strategy. However, the identification of race as a primary concern, and ultimately more important than other marginalized identities, was problematic. I argue that the district's decision to value race over gender and sexuality inhibited teachers from learning valuable skills in terms of understanding how to address issues of bullying when LGBT youth are involved, and learning what to do when cases of bullying based on perceived or actual gender identity/expression or sexual orientation occur, in addition to understanding what issues LGBT youth face in school.

Participants referred to efforts to improve equity in their schools. When asked during follow up questions to explain the equity process in more detail it became evident that race was a primary concern for improving faculty and student outcomes in the district. Their comments regarding the salience of race in the school district contrasted with the fact that only five participants stated that race was a bullying issue in their school. There were clear benefits to focusing on race and equity within the district, such as improved diversity in the schools among teachers and students, in addition to mandatory training/meetings to improve faculty awareness of issues pertaining to race in the school. However, there were multiple unintended consequences of this approach, namely the exclusion of other important identities such as gender and sexuality, as described by several participants. Across all five schools, teachers mentioned the focus 
on race through equity, and the challenges with this approach. At Central High, Caleb talked extensively about his frustrations with isolating race. Caleb described how he tries to bring other identities into the conversation at professional meetings but is discouraged against doing so: "I try and talk about intersectionality and our queer students of color especially and they are like 'oh we have to isolate race' [...] but we also have to name gender and sexuality as things that people suffer oppression around.” Michael, another teacher at Central High, gave a very honest account of the role of race in the school district, expressing their frustration with the current system:

We worry more about racial tension then we do sexual orientation. So race is always at the top because that's what the district is pushing. So clearly what happens is the principals get a message, 'this is what we're doing', so other things get forgotten. So we're constantly brought back to race but we don't include then other things which might be more useful to look at, you know, like gender, gender in general but then bullying, intimidation.

Michael's comment draws attention to two key issues discussed in previous studies, the role of the district in problem framing, and the influence that district priorities have on school administrators. As expressed by Coburn, Matia, and Choi (2013), district policy determines teacher expectations regarding instruction, as well as what information and messaging is passed on to administrators and teachers within a school district. It is apparent through Michael's response that sexual orientation is not as high of a priority as race, and that other priorities get lost as administrators try to focus their attention on the district's priority. In addition to placing the focus on race and inadvertently drawing attention away from issues of bullying among LGBT youth, the amount of time devoted to race and equity during professional meetings further minimizes the amount of time teachers and administrators had to focus on student issues outside of racial inequities, 
hindering bullying prevention among LGBT youth. This seems to be particularly salient for teachers at Central High. Ryan, who teaches at Central, recalled the amount of equity meetings that teachers engage throughout the year, stating that the entire staff meets once a month for equity training. At Dover High, Patricia explains how her school takes this approach even further: "we're required to meet as a whole school four times per year as a school $[\ldots]$ we're scheduled to meet eight times a year and we hold meetings and kind of workshops about ways to improve equity and inclusion and they come in all different flavors." Patricia later went on to discuss the content of their workshops, which included conversations and activities geared toward understanding personal racial biases that may affect how teachers interact with or perceive their students.

Prior to their discussion of equity, when asked if they ever talk to other teachers regarding bullying prevention strategies in the classroom, Patricia described how most of staff time was devoted to discussions around being an equitable teacher, which mostly incorporated discussions about race, as well as mentioning that there were few discussions of bullying within the school. The use of messaging regarding what type of teacher she should be further illustrates the way that district policy creates expectations around teacher behavior in school, either how/what they teach or the type of teacher that they choose to be. At Eastpointe High, there were differences among teachers regarding the focus on race in the school. For Eric, the equity trainings and meetings were very helpful and successful, though he did wish that LGBTQ issues were incorporated into the discussion:

As we're going into equity, diversity and race talks, I wish the LGBTQ talks were in there because it's all a matter of segregation, it's all a matter of differentiating $[\ldots]$ the biggest thing within the equity that we've been working on is taking that 
fear away of having that conversation and I think a lot of bullying in my experience comes from fear of the unknown.

Eric's comment was very similar to Caleb's, who recognized that was important to not only look at race but to take an intersectional approach and try to address all of the marginalized groups represented in the student body. In contrast, George, who also teaches at Eastpointe High, presented a contrasting viewpoint, critiquing the effectiveness of the equity trainings, and also revealed his skepticism and concerns with trying to take a similar approach to training on LGBT youth:

I hear colleagues of mine say things that are really derogatory about what we do and how no one can tell them they have white privilege and it doesn't exist and I don't think they really get a lot from these mandatory meetings and so I wonder if it would go the same way if we have a lot of mandatory meetings about how to be an ally to LGBTQ youth.

George later goes on to talk about how they value training and believed that the training had been very effective for them in forcing them to confront any unconscious racial biases they may have as an educator. Despite George's success with the trainings, it was evident that teachers had very different experiences when it came to confronting and isolating racial issues in their school. George's concern about translating mandatory meetings about race to LGBT youth was valid, but more important provides additional support for the need to ensure that all teachers receive adequate training on all the protected groups in their school's anti-bullying policy, and are held accountable for their behavior.

Mark, an administrator at Avenue High, provided insight on how the district focus on race effected administrators differently than teachers. After discussing that isolating race and examining whiteness were critical aspects of the district's equity policy, Mark 
also mentioned who support groups were created for administrators: “[ $[\ldots]$ the district says we know we need to support our diverse administrators and providing time to support each other $[\ldots]$ build friendships and networks." The support groups were created for administrators at the district-level but not for teachers.

Mark did go on to discuss how, even though teachers were not required to form support groups, there were teachers who created a support group around sexual and gender identity. When describing the groups in more detail, Mark stated:

it's like a support piece where the district says we know we need to support our diverse administrators and providing time to support each other collegially and build friendships and networks, it's important because a lot of white heterosexual principals just do that naturally.

Even though the district was aware that diverse administrators needed spaces to develop bonds with one another and support each other as colleagues, the affinity groups were only organized for administrators. Although Mark did support the group that met around sexual identity and supported each other at lunch, he did not required teachers to create these groups in his school. Mark's comments not only illustrated the amount of time and effort devoted to race within the district, but ultimately reiterate the role of the district and school administrators in problem framing and the factors that influence sense-making among teachers (Coburn 2001; Coburn 2004; Coburn 2006). If the district were to require school administrators to create affinity groups among teachers, it would enable teachers to create networks and communicate more regarding their experiences and even their role in bullying prevention.

Participant interviews illustrate that race and racial equity were repeatedly framed by the district as problem areas and the use of staff time through professional 
development and training reiterated that message. Similar to the findings of Marz and Kelchtermans (2013), Michael, Patricia, Ryan, and Caleb demonstrate that teachers interpret messages differently. At Central High, although participants Ryan and Caleb both mentioned the focus on race as limiting, they later discussed how they employed community building strategies in their classrooms and try to send the message to their students that it is important to respect for all students regardless of their identities. While they did support the racial equity work in the school, it did not prevent them from creating an inclusive classroom environment for LGBT youth. In contrast, Patricia's experience illustrates a potential consequence of working in an environment that is less supportive of discussing all forms of bullying. Allowing for effective and frequent discussions of bullying would have allowed teachers to learn more about all forms of bullying, including cyberbullying. In the following subsection, I examine challenges with reporting cyberbullying in their school.

\section{Challenges to reporting: cyberbullying}

With more and more students using the internet, and having mobile devices, teachers are constantly challenged to be more adept to the ways that youth can engage in bullying behaviors (Underwood and Rosen 2011; James et al. 2008). In addition to issues pertaining to race and the conflict that focusing on race created when trying to interrupt bullying based on LGBT youth, teachers also struggled to feel confident in their ability to address issues related to cyberbullying. Cooper and Blumenfield (2012) demonstrated that cyberbullying is an important issue for LGBT youth. In their study, the majority of LGBT youth in the reported cyberbullying based on their sexual identity, and roughly a 
quarter of the LGBT youth in the study reported isolating themselves from friends, lower grades in school, and being afraid to go to school because of cyberbullying (Cooper and Blumenfield 2012). These findings are important to understanding why teachers' experiences with trying to address cyberbullying pose challenges for LGBT youth.

At the state and district levels, cyberbullying was included in definitions of bullying and harassment, but the school handbook did not discuss cyberbullying when defining harassment or bullying. I am not suggesting that the existence of cyberbullying created a structural barrier for teachers. I argue that the cyberbullying poses challenges to teachers protecting LGBT youth. The difficulties preventing cyberbullying, inability to know when it occurs, and issues related to disciplinary action related to cyberbullying. Although there were no accounts of cyberbullying specifically against LGBT youth in the schools, it is likely that since cyberbullying is an increasing form of bullying, that LGBT youth are also being bullied through social media and the internet. Consistent with the existing literature, teachers and administrators frequently referenced cyberbullying as a challenge to reporting and addressing bullying in their school (James et al. 2008). In addition to the prevalence of cyberbullying, teachers commented that they were unaware of which cyberbullying incidents had occurred, and were very limited in their ability to be proactive in preventing incidents from occurring. Across the schools, participants at Bayview High discussed cyberbullying most frequently. While teachers and administrators did discuss prevention campaigns against cyberbullying, it was also evident that cyberbullying posed several challenges in terms of prevention. Emily and Nathan both describe how they are limited as teachers in their ability to address issues related to cyberbullying. The primary reason they noted was that teachers cannot 
intervene in an instance that they did not see. Nathan talked about how student surveys revealed that most of the bullying had moved online, out of teachers' reach due to restrictions on having a social media account. Emily describes her frustration with cyberbullying below:

Because I feel like because so much bullying has moved online, there's way more silence around it, it's less visible [...] those types of stereotypical old school bullying tactics that were used even before the internet even existed were so blatant and obvious whereas now the students so deeply have their own hidden culture that it's so much harder to even know what exactly is going on $[\ldots]$

In terms of reporting, the movement of bullying from person-to-person to a heavy online presence had teachers and administrators grappling with how to be aware of incidents, let alone address them before they occurred and prevent them before they happened. Sarah, an administrator at Avenue High, discussed how cyberbullying was very difficult to address on the discipline side. To try and combat this, Sarah described how she tries to tell students to take a screenshot and send it to the administration, a tactic that had recently worked to resolve an incident involving two students on a social media platform. Sarah's example exemplifies one of the many ways that teachers and administrators navigate protecting their students from bullying. In the following section, I demonstrate how teachers leveraged existing social networks to addressing bullying.

Taken as a whole, assessments of the challenges faced by teachers and administrators exposed multiple barriers to not only implementing the schools antibullying policy, but also using the policy to protect LGBT youth from bullying. The district framing of race as a problem created additional structural barriers to effectively implementing the anti-bullying policy and protecting LGBT youth as mandatory equity trainings, and pressure to focus on race further reduced the time and resources that 
teachers had to devote to LGBT youth and bullying. Without proper framing of LGBT youth as a priority in the district, there were barriers to forming networks around knowledge of LGBT youth issues and preventing/addressing bullying of LGBT youth. Teachers were not confident in their ability to intervene and address cyberbullying, which was largely a reflection of the lack of guidance in the policy regarding addressing cyberbullying.

Despite these structural limitations, some teachers were able to create communities in their classroom, bring LGBT youth and awareness of LGBT youth issues to the forefront of developing their classroom environments. Teachers at Bayview High School and Central High School provided the best examples of community-building, through the creation of classroom rules that prohibited oppressive language, and classroom activities that promoted comradery and discouraged conflict. The administrative support of both the district and school administrators at Avenue High School and Bayview High School demonstrated the importance of administrators setting the tone for the importance of protecting LGBT youth and how the leadership from the top down has a significant impact on the beliefs and abilities of teachers and administrators to implement an anti-bullying policy. The actions of a few teachers and administrators serve as examples of how to protect LGBT youth but also as critical reminders of the importance of providing teachers and administrators with the appropriate structural supports enabling them to effectively implement school antibullying policies. In the following chapter, I consider the findings of this study in the context of the existing literature on school policy, summarize the results, and provide suggestions for future research. 


\section{DISCUSSION}

In the current study extends the existing literature on anti-bullying policy, by analyzing the relationship between anti-bullying policy implementation and the protection of LGBT youth from bullying. Analyses of teachers' and administrators' perspectives of an anti-bullying policy revealed that the policy created barriers to implementing the anti-bullying policy and protecting LGBT youth from bullying. The state and district language framing bullying as a disciplinary issue posed challenges to teachers trying to address bullying more holistically, and also created barriers for all teachers and administrators in failing to outlining procedures for preventing bullying. Although the policy was readily available through the state and district websites, each school varied in its approach to making the policy available to their staff and students, in addition to their understanding of the policy language, and protocols from reporting.

Contrary to my initial presupposition, the surrounding community's commitment to LGBT youth did not translate into a supportive district or school environment for LGBT youth. This finding adds to the literature on the role of communities in shaping school environments, which suggests that the norms, values and demographics of a community have an impact on the school environment (Coleman 1961; Booth and Gerard 2014). It is possible that for the current study, even though the surrounding community was supportive of the LGBT population, that the level of support was not uniform across each region within the city. For example, both Avenue High School and Bayview High School were model schools in the district, with administrations that supported LGBT youth, and an active QSA student group that was often incorporated in the schools' bullying prevention programs. The commitment of these administrations towards LGBT youth, 
and the efforts of their schools, may in fact reflect the smaller community surrounding their particular schools. For the other schools, it may be the case that community surrounding Eastpointe High, Central High, and Dover High are less welcoming of the LGBT community, which could explain some of the differences between schools in terms of their commitment to LGBT youth. In addition, factors such as race seemed to be more salient for Central High School, which was a minority white school, and Eastpointe High School, which had a larger minority student population compared to Bayview High and Avenue High School. Although there were differences across schools, there were clear structural barriers that affected every participant regardless of location. Training was by far the significant structural issue within the district.

\section{The Anti-Bullying Policy and Problem Framing in the District}

Most consistent with the findings of Coburn, Matia, and Choi (2013), the antibullying policy itself, was the primary source of problem framing within the district. The present study contributes to the literature by providing an account of one of the few districts in the United States that does enumerate sexual orientation and gender identity in the anti-harassment policy, a factor that Kull et al (2015) demonstrate is related to students' likelihood of reporting instances of bullying in their school. While providing this level of inclusion has been associated with increased reporting of incidents by students, in the current study, one challenge was that the district policy essentially framed bullying as a disciplinary issue. The state's conceptualization of bullying as a disciplinary problem was reinforced at the district level, as evident by the language in state law, district policy, and the district handbook. In combination with the variation in availability 
of the district handbook on school websites, it makes sense that participant accounts demonstrated variation not only in terms of understanding bullying, but also in regards to LGBT youth specifically, and the measures taken by teachers to intervene if and when incidents did arise.

Previous research demonstrates that the competence and self-efficacy of teachers and administrators has a significant affect on their likelihood of intervening when bullying occurs in their school (McCabe et al. 2013; James et al. 2008). The results of the current study revealed that teachers and administrators differed in their experiences with training, and understanding the policy. There were also notable differences across schools in terms of conceptualizing bullying as an issue, and approaches to bullying prevention. Administrators held very different beliefs about the explicit language of the policy, some administrators were very aware of the protected classes, and all of the forms of bullying and harassment that were not tolerated (i.e. cyberbullying). Across all five schools, teacher knowledge of the policy varied greatly. Teachers were very candid in expressing their lack of understanding for not only how the policy was enforced at their school, but also their individual role in enforcement. Teachers often stated that if they would channel incidents up to the administration, but few teachers were aware of what happened after they got the administration involved. Teachers were also less knowledgeable of exactly who to go to when an incident did occur. Their lack of knowledge contrasted significantly with that of administrators, who knew it was their responsibility to handle bullying cases, and stated that they would either use discipline exclusively, or a combination of discipline and restorative justice. The gap between teacher and administrator knowledge and competence in terms of policy enforcement is problematic. 
Greytak and Kosciw (2013) indicated that knowing an LGBT student, being aware of general bullying or bullying specific LGBT youth, and their comfort level in terms of intervening are predictors of teachers' likelihood of intervention (Greytak and Kosciw 2013). Thus, if the administration is not passing on information to teachers and helping to build their competence in intervening, incidents may go unreported or unresolved.

Regarding training, the results of the present study reinforce the need to have training, as teachers and administrators across each school reported a lack of training, lack of knowledge of trainings that did exist, and very limited exposure to training specific to LGBT youth. Discrepancies within schools demonstrated that while some teachers did report having training, and could discuss how the district provided training for new teachers, other teachers had never been trained and had no knowledge of district trainings. Some teachers did vaguely recall being exposed to the anti-bullying policy at a staff meeting, and several teachers were aware that the policy was available in the student handbook. Remarkably, one teacher did confront the administration about the lack of training, but was directed toward using their professional leave time to attend an optional training. The results of teacher training provide greater support for the importance of mandatory trainings for teachers and administrators, as well as the importance of providing training on not only the policy but LGBT youth as well. The findings of the current study reinforce those of Corbun, Matia, and Choi (2013) by illustrating that in the absence of adequate policy guidelines for training, bullying was not framed as an issue across all schools.

Results of the present study indicate that when framing of bullying did occur, it resulted in interpretations of bullying as a disciplinary issue. The effect of framing 
bullying as a disciplinary problem was predominately displayed in administrators' and teachers' descriptions of the policy as a zero-tolerance policy. This language was then applied to the classrooms for some teachers, mostly those at Bayview, which had limitations. A consequence of perceiving bullying as an issue to be dealt with through discipline was evidenced in the overwhelmingly reactive approaches to bullying within the schools. The majority of teachers described approaches that involved immediately shutting down the behavior, either in their classrooms or in the hallways, and then informing the administration or writing the student up through their system. Few preventative approaches were mentioned, Avenue High and Bayview High were the only two schools that mentioned having an anti-bullying programs, but the majority of schools did reference the restorative justice program at their school. Restorative justice is a great example of using reconciliation, conflict mediation, and teaching students how to effectively communicate and try to resolve bullying themselves when it does occur. The use of strategies such as restorative justice should be incorporated into the language of anti-bullying policies at the district level to provide guidance and create explicit protocols or addressing bullying that do not result in only disciplinary consequences for students who are bullying other students.

The focus on reactive approaches to bullying is problematic, not only because it does not ensure that bullying will not continue, but teachers and administrators had not received proper training on their school's anti-bullying policy. Their knowledge of the policy, competence in intervention, and belief in the policy itself are integral aspects of their decisions to actually step in and address bullying when it does occur (Marz and Kelchtermans 2013). The lack of consistent training, awareness, and preventative 
approaches is even more alarming, considering that the state policy encourages districts to create training programs for students and all school employees that are geared toward the prevention of bullying, in addition to creating taskforces to implement those prevention programs. Unfortunately, at the district level, this language was lost. Instead, the district policy included recommendations related to increased visibility through signage (i.e. posters, etc.), as well as informing students about definitions, reporting, and consequences. All of these were examples of evidence-bases strategies that the district encouraged schools to employ.

What Works? Perspectives on Bullying Prevention, Policy Effectiveness, and Strategies for Prevention

One of the more substantial contributions this study makes to the existing literature is understanding how teachers and administrators perceive their school's antibullying policy. Several studies have evaluated the overall effectiveness of a school antibullying programs, mainly through pre and post-test measures of teacher/administrator competency, as well as changes to student reports or experiences of victimization (Flygare, Gill, and Johansson 2013; James et al. 2008). In terms of anti-bullying policy, and LGBT youth specifically, there are few studies that address the outcomes of the policy itself. This is largely due to the limited amount of studies that have studied policy implementation more broadly. The most comprehensive study of LGBT youth, antibullying policy, and implementation, comes from GLSEN, and includes measures of compliance between state, and district laws, policies, and the experiences of students within districts that did and did not include protections for LGBT youth (Kull et al. 2015). Their findings demonstrated that few districts require professional development 
and training for school staff when it comes to the policies themselves, let alone bullying and/or harassment specifically. My study supports their findings, but also adds to their study in providing the administrators' and teachers' perspectives on an anti-bullying policy.

Results of participant interviews revealed that both teachers and administrators believed the policy itself was insufficient, recommending a balance between student-led programs, education, training, and the policy. At the schools with well-supported LGBT student-led groups, student involvement in training students, and teachers, on the importance of understanding the issues that LGBT youth face in terms of bullying were commonly discussed. In the schools where teachers either expressed greater frustration with their school administrators, or less awareness of LGBT youth (i.e. presence of LGBT youth, GSAs/QSAs, or training on LGBT youth and bullying prevention), teachers discussed increased training, and better awareness of the policy as important areas for improvement. Surprisingly, even though responses from teachers at each school illustrated inconsistencies in training, knowledge of the policy, and reporting procedures, teachers and administrators at Bayview and Avenue High expressed greater support for the policy's ability to prevent bullying. These differences across schools may be due to both the visibility and awareness that administrators have of LGBT youth. Since the population of LGBT youth at each school is relatively random, these findings provide additional support for the role of administrator training, as studies demonstrate that training can increase administrators' awareness of LGBT-based harassment and bullying (Greytak, Kosciw, and Boesen 2013). 
In terms of the existing policy structure within the district studied, not all teachers and administrators were aware that the school's policy was included in the student handbook. Not one teacher reported reading the policy on the state or district website, and some schools did not offer a link or information about the student handbook and/or district anti-bullying policy on their school's website. If the policy is available, but teachers and administrators are not fully aware of its location, it raises additional concerns in terms of district accountability. Should districts be responsible to ensuring that all school administrators are knowledgeable of the ways for teachers to access the state law on bullying, and policies at the district level? It may be useful for school districts to develop systems for keeping track of administrator training, as well as confirm that schools have the handbook, and anti-bullying policy, readily available on their school's websites. Based on participant responses, accountability at the district level, but also for administrators and teachers, is essential to guarantee that staff are familiar with the policy. Teacher Advocacy, Social Networks, District Priorities, and Cyberbullying

Existing studies suggest that teachers play a significant role in preventing bullying, and also in protecting LGBT youth (Frey et al. 2011; Doll et al. 2011; Grossman et al. 2009). Positive teacher-student relationships are vital to ensuring that students feel safe in the classroom and also feel confident that their teacher is available to and willing to help them if they experience bullying (Doll et al. 2011). In the current study, I demonstrated how teachers advocated for LGBT youth through communitybuilding techniques in their classrooms, support from their administrators, and teachers' use of social networks. Discussions of the resources they used to prevent bullying in their 
classrooms reinforce the importance of the teacher-student relationship, and provide positive examples for other teachers attempting to address issues of bullying based on both sexual orientation and gender identity. Teachers described community-based approaches to interrupting bullying, such as creating classroom rules that prohibit homophobic, racist, or other oppressive remarks. Following the literature both on bullying, and classroom leadership strategies, the creation of formal rules, coupled with informal instruction, are effective tactics to establish a sense of community, but also set very clear expectations regarding student behavior (Frey et al. 2011). Several teachers described this approach, with one teacher going as far as requiring students to conduct research on the oppressive remark they made in class. Teachers also used relationship building techniques to encourage students to develop strong relationships with one another but and their instructor. Although this is an informal form of instruction, it serves to encourage students to see one another as members of a community. Following Rodkin and Gest (2011) the community-based efforts used by some teachers could have resulted in less of a hierarchy within the classroom, which is believed to be associated with less aggression among students, and less instances of peer victimization. Teachers are ultimately able to affect the relationships that students have with their peers, through the way that they structure their classrooms, in addition to the instructional and emotional support that they provide their students (Rodkin and Gest 2011).

The level of commitment that some teachers displayed in their classroom was in part based on individual teacher beliefs regarding bullying, and the anti-bullying policy itself. Both teachers and administrators stated that the policy aligned with their beliefs. Previous literature has attributed congruence between beliefs about instruction and the 
policy itself to result in more positive attitudes toward a school policy, ultimately influencing whether or not teachers choose to implement new school reforms, compared to teachers who did not share similar beliefs about instruction (Marz and Kelchtermans 2013). In addition to teachers who chose to create classroom rules, zero-tolerance approaches to the classroom were also used, which reflects the language in the antibullying policy. For these teachers, disciplinary action occurred first, either through verbally shutting down the incident, to writing the student up for a referral. For other teachers, verbal interruption was the only strategy taken, both in the classroom and in the hallways. In some cases, teachers did try to avoid more punitive measures and involved school counselors or the restorative justice coordinator. These efforts are indicative of using a scaffolding technique, with the overall objective of providing both education and support for students before or after incidents occur (Frey et al. 2011).

Even though teachers did achieve a level of success in terms of creating safe spaces in their classrooms, it was apparent in their reports that not all teachers were using the same techniques. As illustrated by Holt et al. (2011), teachers vary widely in their attitudes and perceptions of bullying. Teachers at Central High demonstrated the greatest degree of variation in teacher understanding of LGBT youth, and their strategies for protecting students in the classroom. One teacher was not at all familiar with the LGBT youth population in their school, while others interacted with LGBT youth frequently, and often tried to advocate for them through requesting additional teacher training. At more than one school, teachers discussed their doubts regarding other teachers respect for students' preferred name or preferred pronoun. One teacher described how their colleagues did not step in to intervene when students were being bullied, and another 
teacher discussed their apprehensive around bring issues related to LGBT youth to their administration. It is possible that teacher frustrations or doubts explained why so few teachers stated that they discussed bullying or the anti-bullying policy with other teachers or staff. The fact that not all teachers intervened when students were bullied was a reflection of the lack of mandatory training for all teachers when it came to the antibullying policy. If intervening when LGBT youth are bullied is left up to individual teachers, some students may be protected when they are in supportive classrooms, but unprotected once they leave. All school districts should be required to ensure that their teachers are trained on anti-bullying policy, are knowledgeable of the issues that affect each protected class in the policy, and feel confident in their ability to step in and intervene when they witness acts of bullying.

One way that teachers could build their capacity for intervening in bullying is through their social networks. In the current study, it was apparent that in the absence of structural supports through training, that teacher discussions of bullying and the antibullying policy ere a vital component of not only sense-making, but also building social capital to implement their school's anti-bullying policy and protect LGBT youth from bullying. Since a new school policy requires teachers to gain new skills and learn new information, where the information comes from has a bearing on whether or not teachers decide to learn the new skills and be involved in implementing the policy (Coburn and Russell 2008; Coburn, Matia, and Choi 2013). In addition to framing bullying as a disciplinary issue, the fact that training for district administrators, teachers, and staff was included in the state law but not the district anti-harassment policy resulted in limited opportunities for teachers interact with administrators and other teachers to learn more 
about the policy and engage in collective sense-making. This finding is particularly important because Coburn, Matia, and Choi (2013) demonstrate that district policy can structure teacher's social networks, offering them opportunities to discuss the policy with other teachers through professional development and trainings. In the absence of these opportunities, the participants in the current study demonstrate limited social capital when it came to understanding their role in policy implementation, and specifically how to help LGBT youth. In the current study, analyses of teachers' discussions of bullying indicated that in the absence of adequate training, teachers' social networks became even more important to increasing their confidence, and helping them to figure out what to do when their students were bullied. For those who did discuss bullying or the policy with other teachers, common areas such as the lunchroom and the teachers' lounge became spaces where teachers could problem solve and receive advice about how to intervene and advocate for students. At Eastpointe High, LGBTQ-identified faculty became chosen experts, providing useful information about LGBT youth for teachers who were not as knowledgeable about issues affecting LGBT youth. For other teachers, meeting with their department colleagues to discuss ways to incorporate LGBTQ issues into the curriculum was a primary example of the many ways that teachers advocated for LGBT youth. Even though teachers were able to build social capital through their social networks and discussions of bullying and/or LGBT youth with colleagues, the sheer lack of training, and knowledge place noteworthy limitations on their abilities.

Further assessments of participant accounts revealed that district priorities posed a substantial risk to teachers' ability to gain the valuable skills necessary to feel competent when it came to LGBT youth and bullying prevention. Race was framed as a primary 
focus area for teachers and administrators across the district. Teachers provided very candid reflections on the role of mandatory equity training, and how focusing on race inadvertently directed staff efforts away from other marginalized identities, such as LGBT youth. From the district to school administration, teachers received very clear messages regarding the need to focus their efforts on racial inequities in the school. Teacher attempts to bring sexuality or gender identity into conversations at equity meetings were unsuccessful, and appeared to be rather discouraging, particularly for faculty who knew of several LGBT students that were experiencing discrimination in school. For that one teacher, it made sense to try and discuss other identities, or include discussions of intersectionality in equity meetings.

Given that LGBT youth includes LGBT youth of color, the resistance over including other identities in equity discussions is rather disheartening. Teachers were very open about discussing the amount of time that was devote to equity within the district, with one teacher claiming that the majority of staff time was devoted to discussions of how to be a more equitable teacher, which included discussions around race. Teachers reported frequent meetings around equity, ranging from multiple times a year to one a month. Although reducing racial inequities in schools is an important issue, the disproportionate amount of time spent focusing on race not only sends a message to teachers that race is more important than LGBT youth issues, but also further restricts what little time teachers have available to receive training, or learn more about LGBT youth. Given that training and self-efficacy can predict if teachers are likely to intervene when youth are bullied, deliberate attempts to diminished the focus on one minority group could result in fewer students being protected from bullying. In addition to 
challenges due to district framing of race as a priority, teachers expressed difficulties with reporting, mostly due to a lack of knowledge of school protocols, but also due to the prevalence of cyberbullying.

Teachers were seldom aware of cyberbullying incidents but frequently acknowledged their limitations in terms of addressing incidents, due to lack of awareness but also because they were not able to monitor students' use of the internet or social media applications. Administrators also expressed their concerns over cyberbullying and often resorted to encouraging teachers and students to get screenshots to serve as evidence of an offense. Although cyberbullying was actually included in the language of the state and district policies, the student handbook did not distinguish between cyberbullying and bullying or harassment. This subtle difference may contribute to teacher and administrator difficulty around reporting. The fact that there were not guidelines for reporting or identifying cyberbullying, reflects the limited knowledge that teachers and administrators have on the differences between cyberbullying and bullying in general, a distinction that researcher suggest is important when trying to understand and address both types of bullying (Underwood and Rosen 2011).

How the District and Schools Advocated for LGBT Youth

Despite the evident challenges, for both teachers and administrators in the study, the lack of adequate training and knowledge of the anti-bullying policy did not prevent schools from finding ways to protect LGBT youth from bullying. The success of the schools included in this study is best displayed in the positive examples of support for the transgender student population. At Bayview High and Avenue High in particular, teachers recalled numerous examples of transgender students' feelings of support at the 
school, and administrative support for gender-neutral bathrooms. Administrators at Bayview also encouraged teachers to ask students their preferred pronoun at the beginning of the year, as well as discouraging teachers from dividing their classrooms by gender. Their efforts are a direct reflection of the district administration, who were commended, both teachers and administrators, for their commitment and dedication to the LGBT student body. Even though some teachers and administrators were less supportive of LGBT youth, the overall sense of support from the top-down within the district serves as a model to other school districts trying to adopt and implement antibullying policies that are inclusive of LGBT students.

Through accounts and analyses of how teachers and administrators interpreted and used anti-bullying policies in their schools, the present study reveals that there is a substantial difference between policy and practice when it comes to anti-bullying policies in high schools. Both individual level and structural level factors had a significant impact on teachers' abilities to advocate for students. At the individual level, teacher and administrator beliefs impacted not only their level of support for the anti-bullying policy, but also the extent to which they chose to implement the policy in their classrooms. Structurally, district priorities and training created a policy environment where teachers and administrators were limited in their ability to effectively implement the school's policy, as well as find ways to protect LGBT youth from bullying. Administrators played a vital role in ensuring that teachers felt supported in their efforts to support their students and create a safe and welcoming environment for all youth. Results of participant interviews at five different schools within one district demonstrate that the relationship between bullying prevention, anti-bullying policy implementation, and LGBT youth is 
complex. As evidenced in the social science and education policy literature, very little information is known about how teachers and administrators are involved in the enforcement of anti-bullying policies. However, results for multiple studies indicate that teachers and administrators play an important role in bullying prevention and providing a safe environment for LGBT youth (McCabe et al. 2013; Kolbert et al. 2015).

I argue that the results of the participant interview data indicate that despite an unsupportive structural environment leaving teachers and administrators without adequate training on bullying prevention, the district's anti-bullying policy, or the prevention of bullying based on sexual orientation or gender identity, teachers still manage to protect their students in a variety of ways. Specifically, the structure of the school affected how and if teachers and administrators intervened, and what methods were used to prevent bullying and to protect LGBT youth. Although the adoption of a state policy created one uniform document outlining how bullying and harassment are perceived and addressed, at each step in the process of policy translation, from state to district to school, understanding of the problem and its solutions were severely altered. Within schools, how a policy was implemented was significantly affected by teachers, who served as advocates in bullying prevention, administrative support, and the students themselves. There were in essence a multitude of barriers to successfully and uniformly implementing a school anti-bullying policy, namely the lack of training, lack of administrative framing of bullying as a problem, the presence of cyberbullying, the lack of social capital, and other marginalized identities, such as race.

Among teachers, social networks proved invaluable in bullying prevention, as several teachers referenced their peers as a source for problem-solving, supporting one 
another as they attempt to protect their students from bullying, and helping them make sense of LGBT youth issues related to bullying. In contrast to the culture of the school, responses from teachers, administrators, and staff highlight the difficulties associated with trying to implement a school anti-bullying policy. In each school there was not a clear consensus on whether or not bullying was a problem. The lack of training, lack of awareness of the policy itself, and focus on reactive approaches to prevention combined to create an environment in each school where the school's anti-bullying policy was being implemented in a variety of ways and interpreted differently within and across occupations.

Regarding LGBT youth, results of the study highlight the importance of naming sexual orientation as a protected class in the language of a policy, and draw attention to the many factors that inhibit teachers from protecting LGBT youth in their schools, including the lack of social networks to discuss bullying and learn about the issues that affect LGBT youth, the district's prioritizing of race over sexual orientation and gender identity/expression, and the inability to intervene in issues related to cyberbullying. Despite evident challenges to policy implementation and the protection of LGBT youth, the present study illustrates how one school district worked tirelessly to try and provide a supportive environment for the students it serves. Each participant spoke very highly of the proactive strategies that were being employed at their institution, ranging from student-led anti-bullying campaigns, to the use of restorative justice techniques to teach students to resolve their differences and avoid disciplinary action. The teachers that participated in the study are exemplary in their commitment to their students and employment of community learning tactics to rid their classrooms from disrespectful 
comments or behaviors. Similarly, the administrators interviewed all spoke of their desire to make sure that all students felt safe in their school, regardless of their sexual orientation or gender identity. The findings in the current study add greatly to the existing literature, which to this point has focused largely on peer interactions in bullying prevention, and has not qualitatively assessed how teachers and administrators perceive the anti-bullying policy environment, and the use of anti-bullying policies as a means to protect LGBT youth in their school. The present study also focuses on LGBT youth, and illuminates several challenges that presumably are faced by school districts across the country, trying to balance protecting all students with making sure that each protected class listed in the policy is in fact protected from harm.

\section{Limitations}

The current study is not without limitations. While the structure of the study could be replicated in other state school districts, the potential findings are not generalizable due to district and school characteristics that are unique to schools in the state. Although this may be a restriction of this study, the policy has been in place for six years, providing a useful opportunity to examine how a longstanding policy is being implemented at the level of secondary schools. The current study can only speak to the experiences of teachers and administrators, as students were excluded from this study. Previous studies have interviewed students to gain their perspectives, which provided useful accounts of perceptions staff and teachers' willingness to intervene, as well as what factors contribute to students feeling supported in their school and community environment, in addition to believing that they can go to a teacher or administrator for help (Grossman et al. 2009; Mishna et al. 2009). The sampling strategy may negatively 
affect the results as obtaining recommendations from principals and teachers to gather additional participants prevent a random sample for the study. In addition, this study provided an assessment of teacher and administrator accounts of their experiences with policy which does not allow for an evaluation of the overall policy or anti-bullying programs. Despite this limitation, the resulting population included 21 participants across five different schools, representing half of the schools in the district and providing a rich sample for this study.

\section{Suggestions for Future Research}

When taken as a whole, my investigation not only contributes to the existing literature by focus on policy implementation and introducing a qualitative account of teachers' and administrators' perspectives on protecting LGBT youth, but also identifies the following questions for future research: 1) How do schools ensure that all protected classes are positively affected by the adoption of a broad-based anti-bullying policy? 2) In what ways are district and school administrators held accountable for the training and competency of their teachers in regards to anti-bullying policy implementation? 3) How do LGBT youth perceive anti-bullying policies as a means to protect them in school? In terms of accountability among administrators, future research should also address unique differences in policy implementation among administrators, such as the differences in messaging, intervention, and perceptions on student behaviors among vice principals, versus principals, and other administrative personnel. Further examination into the use of anti-bullying policies in the United States will ensure that schools and districts are able to effectively protect their students regardless of their identity. 


\section{CONCLUSION}

The current state of bullying prevention in the United States is marked with challenges. Despite evident gains in bullying prevention, as expressed in declining bullying rates and national prevention strategies, few studies have explored how antibullying policies protect vulnerable groups, such as LGBT youth. The present study sought to understand the relationship between bullying prevention, anti-bullying policy implementation and the protection of LGBT youth. Results from the current study confirmed that anti-bullying policies are a vital piece to protecting LGBT youth from bullying. However, participant interviews illustrated that without adequate resources, teachers were unable to be an effective part of the process of implementing school policy. Participant reflections revealed the complex nature of the school policy environment, and highlighted the challenges that teachers and administrators faced with trying to integrate their school's anti-bullying policy into the fabric of their schools. Comparisons both within and across schools in a large, urban school district illuminated substantial differences in how both teachers and administrators conceptualize bullying, interpret the school's anti-bullying policy, and reconcile their own beliefs with the school's protocol for addressing bullying. Nonetheless, participant accounts draw attention to the increased need for training, evaluation, and accountability in terms of ensuring that every staff member is equipped to intervene, and prevent instances of bullying when they do arise.

LGBT youth present unique challenges in terms of bullying, as the existing literature demonstrates how limited policy protections, and school support, create barriers for students and often diminish their feelings of safety and belonging while in school (Grossman et al. 2009; Mishna 2009). The results of this study have several implications 
for school policy. Regarding differences between the state law, district policy, and implementation of the policy at the school level, it is important for states, districts, and schools to effectively coordinate with one another to develop and implement the antibullying policies. States policy makers could convene with district-level personnel to make sure that schools have the appropriate resources for training their employees on the policy. Anti-bullying policies should include explicit language requiring school districts and schools to have mandatory training for all employees. Every teacher and staff member needs to be trained on the anti-bullying policy, and any specific information regarding the prevention of bullying against LGBT youth. Professional development meetings serve as useful sites for allowing teachers and administrators to receive adequate training on school policies and would also promote the creation of social networks to allow teachers to become confident in the procedures for addressing bullying in their school. In terms of training on LGBT youth, community organizations with expertise in the issues that LGBT youth face and their risk factors associated with bullying would be ideal stakeholders when developing training modules for teachers and administrators. Additional guidelines for the investigation and prevention of cyberbullying would also be beneficial and hopefully increase reporting for cyberbullying against LGBT youth and allow teachers to feel better equipped to address cyberbullying in their schools. Instead of integrating bullying into existing training or prevention programs, new programs for bullying prevention should also be required to make sure that teachers and administrators are aware of the nuances of bullying and how approaches to addressing and preventing bullying may differ from other approaches to manage student behavior in the school. School-wide approaches to bullying prevention (i.e., 
student-led anti-bullying programs, campaigns throughout the year, and collaborations across schools) are useful strategies to employ, and are an important aspect of improving the school's culture of acceptance toward LGBT youth, and sending a message that bullying is not prohibited in the school, but these programs cannot serve as a substitute for effective training of all staff, and making sure that everyone is aware of the language in their school and district anti-bullying policies.

For LGBT youth in particular, the current study demonstrated that the individual actions of a few teachers can provide a supportive classroom environment for LGBT youth and encourage youth who do not identity as LGBT to treat their classmates as members of community. Complete coverage in terms of having all states and districts in the U.S. creating anti-bullying policies and including both sexual orientation and gender identity/expression as protected classes is an important step toward ensuring that proactive educators are not the only ones involved in bullying prevention for LGBT youth. Effective training and opportunities for networking with other teachers and administrators can help to encourage teachers and administrators who may not be as motivated to believe in the value of protecting LGBT youth from bullying in their school. My hope is that this study becomes apart of a long list of future studies aiming to further examine the intricacies of policy implementation in efforts to enhance the school environment for LGBT youth and make all schools across the United States places where students, regardless of their sexual orientation or gender identity, are welcomed and feel safe in the classrooms and in the hallways. 


\section{REFERENCES}

Anagnostopoulos, Dorothea, Nicole T. Buchanan, Christine Pereira, and Lauren F. Lichty. 2009. "School Staff Responses to Gender-Based Bullying as Moral Interpretation an Exploratory Study." Educational Policy 23(4):519-53.

Bauman, Sheri, Ken Rigby, and Kathleen Hoppa. 2008. "US Teachers' and School Counsellors' Strategies for Handling Bayview Highullying Incidents." Educational Psychology 28(7):837-56.

Bauman, Sheri and Adrienne Del Rio. 2006. "Preservice Teachers' Responses to Bullying Scenarios: Comparing Physical, Verbal, and Relational Bullying." Journal of Educational Psychology 98(1):219-31.

Benford, Robert D. and David A. Snow. 2000. "Framing Processes and Social Movements: An Overview and Assessment." Annual Review of Sociology 26:611-39.

Booth, Margaret Zoller and Jean M. Gerard. 2014. “Adolescents' Stage-Environment Fit in Middle and High School: The Relationship between Students' Perceptions of Their Schools and Themselves." Youth \& Society 46(6):735-55.

Boulton, Michael J. 1997. "Teachers' Views on Bullying: Definitions, Attitudes and Ability to Cope." British Journal of Educational Psychology 67(2):223-33

Bourdieu, Pierre. 1986. "The Forms of Capital." in Handbook of Theory and Research for the Sociology of Education. New York: Greenwood. Retrieved (http://www.marxists.org/reference/subject/philosophy/works/fr/bourdieu-formscapital.htm).

Button, Deeanna M., Daniel J. O'Connell, and Roberta Gealt. 2012. "Sexual Minority Youth Victimization and Social Support: The Intersection of Sexuality, Gender, Race, and Victimization." Journal of Homosexuality 59(1):18-43.

Cahill, Sean and Jason Cianciatto. 2004. "U.S. Policy Interventions That Can Make Schools Safer." Journal of Gay \& Lesbian Issues in Education 2(1).

Cassidy, Wanda, Karen Brown, and Margaret Jackson. 2012. "'Under the Radar': Educators and Cyberbullying in Schools." School Psychology International 33(5):520-32.

Charmaz, Kathy. 2006. Constructing Grounded Theory: A Practical Guide through Qualitative Analysis. 1 edition. London; Thousand Oaks, Calif: SAGE Publications Ltd.

Cianciotto, Jason and Sean Cahill. 2012. LGBT Youth in America's Schools. Ann Arbor: TheUniversity of Michigan Press.

Coburn, Cynthia E. 2001. "Collective Sensemaking about Reading: How Teachers Mediate Reading Policy in Their Professional Communities." Educational Evaluation and Policy Analysis 23(2):145-70.

Coburn, Cynthia E. 2004. "Beyond Decoupling: Rethinking the Relationship Between the Institutional Environment and the Classroom." Sociology of Education 77(3):211-44.

Coburn, Cynthia E. 2006. "Framing the Problem of Reading Instruction: Using Frame Analysis to Uncover the Microprocesses of Policy Implementation." American Educational Research Journal 43(3):343-79. 
Coburn, Cynthia E. and Jennifer Lin Russell. 2008. "District Policy and Teachers' Social Networks." Educational Evaluation and Policy Analysis 30(3):203-35.

Coburn, Cynthia E., Willow S. Mata, and Linda Choi. 2013. "The Embeddedness of Teachers' Social Networks: Evidence from a Study of Mathematics Reform." Sociology of Education 86(4):311-42.

Coleman, James, S. 1961. The Adolescent Society; the Social Life of the Teenager and Its Impact on Education,. New York Free Press of Glencoe.

Coleman, James, S. and Thomas Hoffer. 1987. Public and Private High Schools: The Impact of Communities. New York: Basic Books.

Coleman, James S. 1988. "Social Capital in the Creation of Human Capital." American Journal of Sociology 94:S95-120.

Cooper, Robyn M. and Warren J. Blumenfeld. 2012. "Responses to Cyberbullying: A Descriptive Analysis of the Frequency of and Impact on LGBT and Allied Youth." Journal of LGBT Youth 9(2):153-77.

Desimone, Laura M. 2006. "Consider the Source Response Differences Among Teachers, Principals, and Districts on Survey Questions About Their Education Policy Environment." Educational Policy 20(4):640-76.

Doll, Beth, Samuel Song, Allison Champion, and Kristin Jones. 2011. "Classroom Ecologies That Support or Discourage Bullying.” Pp. 147-58 in Bullying in North American Schools. New York and London: Taylor and Francis.

Eccles, Jacquelynne S. et al. 1993. "Development during Adolescence: The Impact of Stage-Environment Fit on Young Adolescents' Experiences in Schools and in Families." American Psychologist 48(2):90-101.

Fetner, Tina, Athena Elafros, Sandra Bortolin, and Coralee Drechsler. 2012. "Safe Spaces: Gay-Straight Alliances in High Schools." Canadian Review of Sociology/Revue canadienne de sociologie 49(2):188-207.

Flygare, Erik, Peter Edward Gill, and Björn Johansson. 2013. "Lessons From a Concurrent Evaluation of Eight Anti-bullying Programs Used in Sweden." American Journal of Evaluation 34(2):170-89.

Frey, Karin. S., Diane Carlson Jones, Miriam K. Hirschstein, and Leihua Edstrom. 2011. "Teacher Support of Bullying Prevention: The Good, the Bad, and the Promising." Pp. 266-77 in Bullying in North American SChools. New York and London: Routledge.

Gallucci, Chrysan. 2003. "Communities of Practice and the Mediation ofTeachers' Responses to Standards-Based Reform." education policy analysis archives 11(0):35.

Goodenow, Carol, Laura Szalacha, and Kim Westheimer. 2006. "School Support Groups, Other School Factors, and the Safety of Sexual Minority Adolescents." Psychology in the Schools 43(5):573-89.

Grace, André P. and Kristopher Wells. 2009. "Gay and Bisexual Male Youth as Educator Activists and Cultural Workers: The Queer Critical Praxis of Three Canadian High-school Students.” International Journal of Inclusive Education 13(1):23-44. 
Greytak, Emily A. and Joseph G. Kosciw. 2014. "Predictors of US Teachers' Intervention in Anti-Lesbian, Gay, Bisexual, and Transgender Bullying and Harassment." Teaching Education 25(4):410-26.

Greytak, Emily A., Joseph G. Kosciw, and Madelyn J. Boesen. 2013. "Educating the Educator: Creating Supportive School Personnel Through Professional Development." Journal of School Violence 12(1):80-97.

Griffin, Pat, Camille Lee, Jeffrey Waugh and Chad Beyer. 2004. "Describing Roles that Gay-Straight Alliances Play in Schools." Journal of Gay \& Lesbian Issues in Education 1(3): 7-22. DOI: 10.1300/J367v01n03_03

Grossman, Arnold H. et al. 2009. "Lesbian, Gay, Bisexual and Transgender Youth Talk about Experiencing and Coping with School Violence: A Qualitative Study." Journal of LGBT Youth 6(1):24-46.

Hatzenbuehler, Mark L. 2011. "The Social Environment and Suicide Attempts in Lesbian, Gay and Bisexual Youth.” American Academy of Pediatrics 127(5):896903.

Hatzenbuehler, Mark L. and Katherine M. Keyes. 2013. "Inclusive Anti-Bullying Policies and Reduced Risk of Suicide Attempts in Lesbian and Gay Youth.” Journal of Adolescent Health 53(1, Supplement):S21-S26.

Hatzenbuehler, Mark L., Michelle Birkett, Aimee Van Wagenen, and Ilan H. Meyer. 2013. "Protective School Climates and Reduced Risk for Suicide Ideation in Sexual Minority Youths." American Journal of Public Health e1-e8.

Holt, Melissa, Melissa Keyes, and Brian Koenig. 2011. "Teachers' Attitudes Toward Bullying." Pp. 119-31 in Bullying in North American SChools. New York and London: Routledge.

Human Rights Campaign (HRC). 2016. "Student Non-Discrimination Act.” Human Rights Campaign. Retrieved March 24, 2016 (http://www.hrc.org/resources/student-non-discrimination-act/).

Ingersoll, Richard, M. 2003. Who Controls Teachers' Work?: Power and Accountability in America's Schools. Cambridge, Mass: Harvard University Press.

Jacob, Susan. 2013. "Creating Safe and Welcoming Schools for LGBT Students: Ethical and Legal Issues." Journal of School Violence 12(1):98-115.

James, Deborah J. et al. 2006. "One School's Experience of Engaging with a Comprehensive Anti-Bullying Programme in the Irish Context: Adolescent and Teacher Perspectives." Pastoral Care in Education 24(4):39-48.

Kann et al. 2014. Youth Risk Behavior Surveillance-United States, 2013. MMWR; 63 (SS4): 1-168. Retrieved 19 May 2015 from http://www.cdc.gov/mmwr/pdf/ss/ss6304.pdf.

Kann, Laura et al. 2009. "Sexual Identity, Sex of Sexual Contacts, and Health-Risk Behaviors Among Students in Grades 9--12 --- Youth Risk Behavior Surveillance, Selected Sites, United States, 2001--2009." Morbidity and Mortality Weekly Report 60(7). Retrieved January 23, 2014 (http://www.cdc.gov/mmwr/preview/mmwrhtml/ss6007a1.htm).

Kennedy, Tom D., Ashley G. Russom, and Meline M. Kevorkian. 2012. "Teacher and 
Administrator Perceptions of Bullying in Schools.” International Journal of Education Policy and Leadership 7(5). Retrieved July 16, 2015 (http://journals.sfu.ca/ijepl/index.php/ijepl/article/view/395).

Kester, K. and Candiya Mann. 2008. Bullying in Washington Schools: Update 2008. Social and Economic Sciences Research Center. Olympia, Washington.

Kolbert, Jered B. et al. 2015. "Teachers' Perceptions of Bullying of Lesbian, Gay, Bisexual, Transgender, and Questioning (LGBTQ) Students in a Southwestern Pennsylvania Sample.” Behavioral Sciences 5(2):247-63.

Kosciw, J. G., Greytak, E. A., Palmer, N. A., \& Boesen, M. J. 2014. The 2013 National School Climate Survey: The experiences of lesbian, gay, bisexual and transgender youth in our nation's schools. New York:GLSEN.

Kull, R. M., J. G. Kosciw, and E. A. Greytak. 2015. From Statehouse to Schoolhouse: Anti-Bullying Policy Efforts in U.S. States and School Districts. New York: GLSEN.

März, Virginie and Geert Kelchtermans. 2013. "Sense-Making and Structure in Teachers' Reception of Educational Reform. A Case Study on Statistics in the Mathematics Curriculum." Teaching and Teacher Education 29:13-24.

Mayberry, Maralee, Tiffany Chenneville, and Sean Currie. 2013. "Challenging the Sounds of Silence: A Qualitative Study of Gay-Straight Alliances and School Reform Efforts." Education and Urban Society 45(307):339.

McCabe, Paul C., Florence Rubinson, Eliza A. Dragowski, and Graciela Elizalde-Utnick. 2013. "Behavioral Intention of Teachers, School Psychologists, and Counselors to Intervene and Prevent Harassment of Lgbtq Youth.” Psychology in the Schools 50(7):672-88.

Meyer IH. 2003. "Prejudice, Social Stress, and Mental Health in Lesbian, Gay, and Bisexual Populations: Conceptual Issues and Research Evidence." Psychological bulletin 129(5):674-97.

Minton, Stephen James, Torunn Dahl, Astrid Mona O’ Moore, and Donnely Tuck. 2008. "An Exploratory Survey of the Experiences of Homophobic Bullying among Lesbian, Gay,

Bisexual and Transgendered Young People in Ireland." Irish Educational Studies 27(2):177-91.

Mishna, Faye, Peter A. Newman, Andrea Daley, and Steven Solomon. 2009. "Bullying of Lesbian and Gay Youth: A Qualitative Investigation.” British Journal of Social Work 39(8):1598-1614.

Marvasti, Amir. 2003. Qualitative Research in Sociology. London; Thousand Oaks, Calif: SAGE Publications Ltd.

Pascoe, C. J. 2013. "Notes on a Sociology of Bullying: Young Men's Homophobia as Gender Socialization.” QED: A Journal in GLBTQ Worldmaking (1):87-103.

Perius, Jessamyn G., Ashley Brooks-Russell, Jing Wang, and Ronald J. Iannotti. 2014. "Trends in Bullying, Physical Fighting, and Weapon Carrying Among 6thThrough 10th-Grade Students from 1998 to 2010: Findings From a National Study." American Journal of Public Health 104(6):1100-1106. 
Peterson, Kent and Terrence Deal. 2011. "How Leaders Influence the Culture of Schools." Pp. 49-60 in Teacher Leadership: The "New" Foundation of Teacher Education. New York: Peter Lang Publishing, Inc.

Poteat, V. Paul, Katerina O. Sinclair, Craig D. DiGiovanni, Brian W. Koenig, and Stephen T. Russell. 2013. "Gay-Straight Alliances Are Associated With Student Health: A Multischool Comparison of LGBTQ and Heterosexual Youth.” Journal of Research on Adolescence 23(2):319-30.

Roeser, Robert W., Jacquelynne S. Eccles, and Arnold J. Sameroff. 2000. "School as a Context of Early Adolescents' Academic and Social-Emotional Development: A Summary of Research Findings." The Elementary School Journal 100(5):443-71.

Russell, Stephen T., Katerina O. Sinclair, V. Paul Poteat, and Brian W. Koenig. 2012. "Adolescent Health and Harassment Based on Discriminatory Bias." American Journal of Public Health 102(3):493-95.

Russo, Ronald G. 2006. "The Extent of Public Education Nondiscrimination Policy Protections for Lesbian, Gay, Bisexual, and Transgender Students: A National Study." Urban Education 41(2):115-50.

Schneider, Margaret S., and Anne Dimito. 2008. "Educators' Beliefs about Raising Lesbian, Gay, Bisexual, and Transgender Issues in the Schools: The Experience in Ontario, Canada." Journal of LGBT Youth 5(4):49-71.

Smylie, Mark A. and Andrea E. Evans. 2006. "Social Capital and the Problem of Implementation". Pp. 187-208 in New Directions in Education Policy Implementation: Confronting Complexity, edited by Meredith I. Hong. Albany, NY: State University of New York Press.

Spillane, James P., Brian J. Reiser, and Todd Reimer. 2002. "Policy Implementation and Cognition: Reframing and Refocusing Implementation Research.” Review of Educational Research 72(3):387-431.

Swearer, Susan M., Dorothy L. Espelage, and Scott A. Napolitano. 2009. Bullying Prevention and Intervention: Realistic Strategies for Schools. New York, NY: Guilford Press.

Swearer, Susan M., Dorothy L. Espelage, Tracy Vaillancourt, and Shelley Hymel. 2010. "What Can Be Done About Bayview Highullying? Linking Research to Educational Practice." Educational Researcher 39(1):38-47.

Terry, Troy M. 2010. "Blocking the Bullies: Has South Carolina's Safe School Climate Act Made Public Schools Safer?" Clearing House: A Journal of Educational Strategies, Issues and Ideas 83(3):96-100.

Thomas, David R. 2006. "A General Inductive Approach for Analyzing Qualitative Evaluation Data." American Journal of Evaluation 27(2):237-46.

Toomey, Russell B., Jenifer K. McGuire, and Stephen T. Russell. 2012. "Heteronormativity, School Climates, and Perceived Safety for Gender Nonconforming Peers." Journal of Adolescence 35(1):187-96.

Underwood, Marion, K. and Lisa Rosen H. 2011. "Gender and Bullying: Moving Beyond Mean Differences to Conceptions of Bullying, Processes by Which Bullying Unfolds, and Cyberbullying." in Bullying in North American Schools. New York, N.Y.: Routledge. 
U.S. Department of Education, Office of Planning, Evaluation and Policy Development, Policy and Program Studies Service. 2011. Analysis of State Bullying Laws and Policies. Washington, D.C.

United States Department of Education. 2013. Student reports of bullying and cyberbullying: Results from the 2011 School Crime Supplement to the National Crime Victimization Survey. Retrieved 19 May 2015 from http://nces.ed.gov/pubs2013/2013329.pdf.

United States Department of Health and Human Services. 2014. "Bullying and Adolescent Health.” Retrieved August 25, 2015 (http://www.hhs.gov/ash/oah/).

United States Department of Health and Human Services. 2014. Bullying and LGBT Youth. Retrieved April 25, 2015 from http://www.stopbullying.gov/atrisk/groups/lgbt.

United States Department of Health and Human Services. 2014. Bullying Definition. Retrieved 19 May 2015 from http://www.stopbullying.gov/what-isbullying/definition/index.htm

United States Department of Health and Human Services. 2014. Lesbian, Gay, Bisexual, and Transgender Health: Overview and Objectives. Healthy People 2020. Retrieved April 25, 2015 from http://www.healthypeople.gov/2020/topicsobjectives/topic/lesbian-gay-bisexual-and-transgender-health/objectives.

Ullman, Jacqueline. 2014. 'Ladylike/butch, Sporty/dapper: Exploring 'gender Climate' with Australian LGBTQ Students Using Stage-environment Fit Theory.” Sex Education 14(4):430-43.

Watson, Laurel B., Kris Varjas, Joel Meyers, and Emily C. Graybill. 2010. "Gay-Straight Alliance Advisors: Negotiating Multiple Ecological Systems When Advocating for LGBTQ Youth.” Journal of LGBT Youth 7(2):100-128. 


\section{APPENDIX A: INTERVIEW GUIDE}

\section{Demographic Questions}

1. What is your current position at the school/district?

2. How long have you worked at this school? How long have you served in this position?

3. How would you describe the composition of your school (i.e. race, size, ses, etc.)?

\section{Bullying}

1. Are you familiar with the Harassment, Intimidation, Bullying and Cyberbullying policy at your school? Can you describe the policy in your own words?

2. How did you learn about the Harassment, Intimidation, Bullying and Cyberbullying policy?

3. Have you ever had training specific to the anti-bullying policy?

4. How is this policy enforced in your school/district?

5. In what contexts have you discussed the policy with other teachers or staff (i.e. staff meetings, professional development seminars, etc.)?

6. What is your role in implementing this policy?

7. Do you think the policy has affected how you teach or interact with students?

8. In your opinion, is bullying a problem in your school?

9. In the past year, are there any instances of bullying that stand out?

Bullying based on perceived sexual orientation/gender identity

10. Are there any transgender or gender non-conforming youth at your school?

11. In the past year, have there been instances of bullying based on perceived sexual orientation at your school that stand out? Cases based on gender identity? How were those cases handled?

12. Are you aware of the inclusion of sexual orientation as a protected class under this policy?

13. In your own words, can you describe how sexual orientation is explained in the policy?

14. In your opinion, can/should this policy protect against harassment based on perceived sexual orientation?

15. To your knowledge, in the past year, have there been teacher trainings or programs in place to prevent bullying based on sexual orientation in the school?

\section{Effectiveness and Change}

16. In your opinion, what should your school do to prevent bullying? What has worked in the past? What strategies have not worked toward the prevention of bullying in your school? 
17. In your opinion, is the HIBC policy an effective policy to prevent bullying in your school?

18. What suggestions do you have for improving the HIBC policy? 


\section{APPENDIX B: RELEVANT LITERATURE}

Table I. Review of Relevant Literature on Teachers/Administrators Role in Bullying and Policy Implementation

\begin{tabular}{|c|c|c|c|}
\hline Journal/Publication & Location & Aim of Study & Methodology \\
\hline $\begin{array}{l}\text { British Journal of } \\
\text { Social Work (2009) }\end{array}$ & Toronto & $\begin{array}{l}\text { Service providers } \\
\text { perspective on bullying } \\
\text { of lesbian and gay youth }\end{array}$ & $\begin{array}{l}\text { Face-to-face } \\
\text { interviews with } 9 \\
\text { service providers }\end{array}$ \\
\hline $\begin{array}{l}\text { Journal of Gay and } \\
\text { Lesbian Issues in } \\
\text { Education (2006) }\end{array}$ & Denver & $\begin{array}{l}\text { School experiences of } \\
\text { suicide prevention for } \\
\text { sexual minority youth }\end{array}$ & $\begin{array}{l}\text { Focus groups } \\
\text { with } 5 \text { self- } \\
\text { identified sexual } \\
\text { minority youth } \\
\end{array}$ \\
\hline $\begin{array}{l}\text { Journal of LGBT } \\
\text { Youth (2009) }\end{array}$ & New York & $\begin{array}{l}\text { LGBT youth perspectives } \\
\text { on past and current } \\
\text { experiences with bullying }\end{array}$ & $\begin{array}{l}\text { Focus groups } \\
\text { with } 31 \text { sexual } \\
\text { minority youth }\end{array}$ \\
\hline $\begin{array}{l}\text { AERA Conference } \\
(2013)\end{array}$ & Florida & $\begin{array}{l}\text { Teacher's perspective on } \\
\text { anti-bullying program } \\
\text { being implemented in } \\
\text { their school }\end{array}$ & $\begin{array}{l}\text { Open-ended } \\
\text { qualitative } \\
\text { interviews with } 9 \\
\text { teachers and } \\
\text { school } \\
\text { counselors }\end{array}$ \\
\hline $\begin{array}{l}\text { Journal of } \\
\text { Interpersonal } \\
\text { Violence (2013) }\end{array}$ & Wisconsin/Indiana & $\begin{array}{l}\text { Parent's perspective on } \\
\text { the bullying reporting } \\
\text { process }\end{array}$ & $\begin{array}{l}\text { Semi-structured } \\
\text { interviews with } \\
11 \text { middle school } \\
\text { parents }\end{array}$ \\
\hline $\begin{array}{l}\text { Educational } \\
\text { Psychology (2008) }\end{array}$ & $\begin{array}{l}\text { Arizona/Australia/ } \\
\text { New Hampshire }\end{array}$ & $\begin{array}{l}\text { How teachers and school } \\
\text { counselors approach } \\
\text { bullying }\end{array}$ & $\begin{array}{l}\text { Online survey } \\
\text { with } 735 \text { teachers } \\
\text { and school } \\
\text { administrators }\end{array}$ \\
\hline $\begin{array}{l}\text { Pastoral Care } \\
(2006)\end{array}$ & Ireland & $\begin{array}{l}\text { Evaluation of the impact } \\
\text { of an anti-bullying } \\
\text { program from teachers } \\
\text { and students }\end{array}$ & $\begin{array}{l}\text { Questionnaire } \\
\text { administered to } \\
200 \text { students and } \\
\text { teachers }\end{array}$ \\
\hline $\begin{array}{l}\text { American Journal } \\
\text { of Evaluation } \\
(2013)\end{array}$ & Sweden & $\begin{array}{l}\text { Effectiveness of specific } \\
\text { anti-bullying programs } \\
\text { and their impact }\end{array}$ & $\begin{array}{l}\text { Mix of } \\
\text { interviews and } \\
\text { questionnaires } \\
\text { with } 1,000+ \\
\text { students and } \\
\text { teachers }\end{array}$ \\
\hline $\begin{array}{l}\text { International } \\
\text { Journal of Critical } \\
\text { Pedagogy (2010) }\end{array}$ & United States & $\begin{array}{l}\text { Effect of cyberbullying } \\
\text { on LGBT and allied } \\
\text { youth }\end{array}$ & $\begin{array}{l}\text { Survey } \\
\text { administered to } \\
444 \text { youth ages } \\
11-22\end{array}$ \\
\hline School Psychology & Canada & Teacher's perceptions of & Semi-structured \\
\hline
\end{tabular}




\begin{tabular}{|c|c|c|c|}
\hline $\begin{array}{l}\text { International } \\
\text { (2012) }\end{array}$ & & $\begin{array}{l}\text { cyberbullying as a } \\
\text { problem }\end{array}$ & $\begin{array}{l}\text { interviews with } \\
17 \text { educators }\end{array}$ \\
\hline $\begin{array}{l}\text { Educational Policy } \\
\text { (2009) }\end{array}$ & United States & $\begin{array}{l}\text { School staff experiences } \\
\text { with gender-based } \\
\text { bullying }\end{array}$ & $\begin{array}{l}\text { Face-to-face } \\
\text { interviews with } \\
10 \text { teachers and } 5 \\
\text { support staff }\end{array}$ \\
\hline $\begin{array}{l}\text { Educational Policy } \\
\text { (2014) }\end{array}$ & $\begin{array}{l}\text { North } \\
\text { Carolina/Texas }\end{array}$ & $\begin{array}{l}\text { Evaluation of policy } \\
\text { implementation based on } \\
\text { principals perceptions of } \\
\text { the policy }\end{array}$ & $\begin{array}{l}\text { Interviews with } 4 \\
\text { school principals }\end{array}$ \\
\hline $\begin{array}{l}\text { Educational Policy } \\
\text { (2006) }\end{array}$ & Nashville & $\begin{array}{l}\text { How perceptions of the } \\
\text { policy system differ by } \\
\text { principals, teachers and } \\
\text { district administrators }\end{array}$ & $\begin{array}{l}\text { Survey } \\
\text { administered to } \\
1,000+\text { teachers } \\
\text { and principals }\end{array}$ \\
\hline $\begin{array}{l}\text { Sociology of } \\
\text { Education (2013) }\end{array}$ & Illinois/California & $\begin{array}{l}\text { Understand how district } \\
\text { policy affects teacher's } \\
\text { social networks }\end{array}$ & $\begin{array}{l}\text { Face-to-face } \\
\text { interviews with } \\
16 \text { teachers and } \\
\text { principals }\end{array}$ \\
\hline $\begin{array}{l}\text { Sociological } \\
\text { Spectrum (2015) }\end{array}$ & California & $\begin{array}{l}\text { Understand teacher's } \\
\text { awareness of bullying } \\
\text { and how they engage } \\
\text { with bullying experiences }\end{array}$ & $\begin{array}{l}\text { Focus groups } \\
\text { with } 96 \\
\text { elementary } \\
\text { school teachers }\end{array}$ \\
\hline $\begin{array}{l}\text { Journal of Early } \\
\text { Adolescence } \\
(2012)\end{array}$ & North Dakota & $\begin{array}{l}\text { To examine how } \\
\text { teacher's beliefs } \\
\text { regarding bullying } \\
\text { influence their and } \\
\text { student's approaches to } \\
\text { intervening in bullying } \\
\text { events }\end{array}$ & $\begin{array}{l}\text { Quantitative } \\
\text { survey } \\
\text { administered to } \\
4003 \mathrm{rd} / 8^{\text {th }} \text { grade } \\
\text { students and } \\
\text { teachers }\end{array}$ \\
\hline $\begin{array}{l}\text { Journal of } \\
\text { Educational } \\
\text { Psychology (2014) }\end{array}$ & $\begin{array}{l}\text { Netherlands, } \\
\text { Finland and } \\
\text { Australia }\end{array}$ & $\begin{array}{l}\text { To assess how teacher's } \\
\text { anti-bullying attitudes } \\
\text { impact the level of } \\
\text { bullying in their } \\
\text { classrooms }\end{array}$ & $\begin{array}{l}\text { Quantitative } \\
\text { questionnaire } \\
\text { administered to } \\
\text { 2,776 elementary } \\
\text { school students } \\
\text { across } 31 \text { schools }\end{array}$ \\
\hline $\begin{array}{l}\text { Teaching } \\
\text { Education (2014) }\end{array}$ & New York & $\begin{array}{l}\text { Understand factors that } \\
\text { may predict teachers } \\
\text { likelihood of intervening } \\
\text { in anti-lgbt harassment }\end{array}$ & $\begin{array}{l}\text { Online survey } \\
\text { administered to } \\
726 \text { middle and } \\
\text { high school } \\
\text { teachers }\end{array}$ \\
\hline $\begin{array}{l}\text { Educational } \\
\text { Psychology (2010) }\end{array}$ & New York & $\begin{array}{l}\text { Examine the role of } \\
\text { teacher preparedness in } \\
\text { bullying interventions }\end{array}$ & $\begin{array}{l}\text { Quantitative } \\
\text { survey } \\
\text { administered to } \\
115 \text { middle } \\
\text { school teachers }\end{array}$ \\
\hline Social Psychology & Greece & Understand adolescent & Focus groups \\
\hline
\end{tabular}




\begin{tabular}{|l|l|l|l|}
\hline Education (2012) & $\begin{array}{l}\text { perspective on how } \\
\text { school context impacts } \\
\text { bullying }\end{array}$ & $\begin{array}{l}\text { with 90 high } \\
\text { school students }\end{array}$ \\
\hline
\end{tabular}




\section{APPENDIX C: STUDY PARTICIPANT CHARACTERISTICS}

in School

Table II. Characteristics of Participants by School, Years in School and Position

\begin{tabular}{r|lll}
\multicolumn{1}{r}{ School } & Participant Name & Position in School & Years in School \\
\hline Avenue High & Sarah & Vice Principal & 2 \\
& Jane & School Psychologist & 14 \\
& Rose & Teacher & 1 \\
& Henry & Teacher & 13 \\
& Zachary & Teacher & 2 \\
& Mark & Principal & 10 \\
& Zoe & Teacher & 4 \\
& & & \\
Bayview High & Anthony & Principal & 3 \\
& Daniel & Teacher & 1 \\
& Nathan & Teacher & 3 \\
& Brenda & Vice Principal & 2 \\
& Emily & Teacher & 2 \\
& Central High & Teacher & 3 \\
& Michael & Teacher & 1 \\
& Ryan & Teacher & 3 \\
& Grace & Teacher & 2 \\
& Caleb & & \\
& & Teacher & 3 \\
Dover High & Patricia & Teacher & 10 \\
& Taylor & Teacher & 6 \\
& Eric & Teacher & 2 \\
& George & Vice Principal & 9
\end{tabular}

